\title{
Compact Fluorescent Retrofits for Incandescent Downlight Fixtures
}

by

William R. Taylor

Compact fluorescent (CF) lamps, which are available as an energy-efficient replacement for ordinary incandescent light bulbs, can be used in a variety of fixture types, including existing incandescent, ceiling-mounted, recessed downlights. However, CF lamps are not shaped or sized identically to the incandescent bulbs being replaced; therefore, light distribution will be changed by these retrofits.

Army facilities have numerous incandescent downlight fixtures that are candidates for compact fluorescent retrofits. The information in this study can provide guidance for selecting appropriate retrofits.

This study examined the photometric effects of installing several types of CF lamps in several types of incandescent downlight fixtures. Test results show that, for shallow depth fixtures, several types of CF lamps perform adequately. For deeper fixtures using nonreflective baffle rings, retrofitting with some CF lamps causes light output from the fixture to drop drastically. CF retrofits also caused a wider pattern of light distribution and increased luminance values. Changes in distribution and luminance need to be considered by Army facility managers contemplating CF retrofits.

\section{For Reference}

Not to be taken from this room 
The contents of this report are not to be used for advertising, publication, or promotional purposes. Citation of trade names does not constitute an official endorsement or approval of the use of such commercial products. The findings of this report are not to be construed as an official Department of the Army position, unless so designated by other authorized documents. 


\section{USER EVALUATION OF REPORT}

REFERENCE: USACERL Technical Report 95/17, Compact Fluorescent Retrofits for Incandescent Downlight Fixtures

Please take a few minutes to answer the questions below, tear out this sheet, and return it to USACERL. As user of this report, your customer comments will provide USACERL with information essential for improving future reports.

1. Does this report satisfy a need? (Comment on purpose, related project, or other area of interest for which report will be used.)

2. How, specifically, is the report being used? (Information source, design data or procedure, management procedure, source of ideas, etc.)

3. Has the information in this report led to any quantitative savings as far as manhours/contract dollars saved, operating costs avoided, efficiencies achieved, etc.? If so, please elaborate.

4. What is your evaluation of this report in the following areas?

a. Presentation:

b. Completeness:

c. Easy to Understand:

d. Easy to Implement:

e. Adequate Reference Material:

f. Relates to Area of Interest:

g. Did the report meet your expectations?

h. Does the report raise unanswered questions? 
i. General Comments. (Indicate what you think should be changed to make this report and future reports of this type more responsive to your needs, more usable, improve readability, etc.)

5. If you would like to be contacted by the personnel who prepared this report to raise specific questions or discuss the topic, please fill in the following information.

\section{Name:}

Telephone Number:

Organization Address:

6. Please mail the completed form to:

Department of the Army

CONSTRUCTION ENGINEERING RESEARCH LABORATORIES

ATTN: CECER-TR-I

P.O. Box 9005

Champaign, IL 61826-9005 


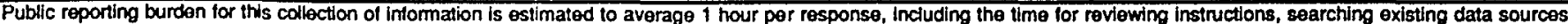

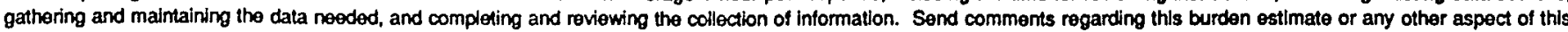

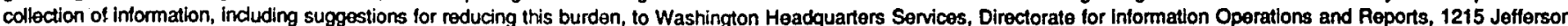
Davis Highway, Suite 1204, Arlington, VA 22202-4302, and to the Office of Management and Budget, Paperwork Reduction Project (0704-0188), Washington, DC 20503.

\begin{tabular}{|c|c|c|c|}
\hline 1. AGENCY USE ONLY (Leave Blank) & $\begin{array}{l}\text { 2. REPORT DATE } \\
\text { August } 1995\end{array}$ & \multicolumn{2}{|c|}{$\begin{array}{l}\text { 3. AEPORT TYPE AND DATES COVERED } \\
\text { Final }\end{array}$} \\
\hline \multicolumn{3}{|c|}{$\begin{array}{l}\text { 4. TITLE AND SUBTITLE } \\
\text { Compact Fluorescent Retrofits for Incandescent Downlight Fixtures }\end{array}$} & $\begin{array}{l}\text { 5. FUNDING NUMBERS } \\
\text { 4A162784 } \\
\text { AT45 } \\
\text { FE-XH4 }\end{array}$ \\
\hline \multicolumn{3}{|l|}{$\begin{array}{l}\text { 6. AUTHOR(S) } \\
\text { William R. Taylor }\end{array}$} & \\
\hline \multicolumn{3}{|c|}{$\begin{array}{l}\text { 7. PERFORMING ORGANIZATION NAME(S) AND ADDRESS(ES) } \\
\text { U.S. Army Construction Engineering Research Laboratories (USACERL) } \\
\text { P.O. Box } 9005 \\
\text { Champaign, IL } 61826-9005\end{array}$} & $\begin{array}{l}\text { 8. PERFORMING ORGANIZATION } \\
\text { REPORT NUMBER } \\
\text { TR } 95 / 17\end{array}$ \\
\hline \multicolumn{3}{|c|}{$\begin{array}{ll}\text { 9. SPONSORING / MONITORING AGENCY NAME(S) AND ADDAESS(ES) } \\
\text { U.S. Army Center for Public Works (USACPW) } & \text { Headquarters, U.S. Army Corps of } \\
\text { ATTN: CECPW-EE } & \text { Engineers (HQUSACE) } \\
\text { 7701 Telegraph Road } & \text { ATTN: CEMP-ET-E } \\
\text { Alexandria, VA 22310-3862 } & 20 \text { Massachusetts Ave., NW } \\
& \text { Washington, DC 20314-1000 }\end{array}$} & $\begin{array}{l}\text { 10. SPONSORING / MONITORING } \\
\text { AGENCY REPORT NUMBER }\end{array}$ \\
\hline
\end{tabular}

Copies are available from the National Technical Information Service, 5285 Port Royal Road, Springfield, VA 22161.

12a. DISTRIBUTION/AVAILABILITY STATEMENT

12b. DISTAIBUTION CODE

Approved for public release; distribution is unlimited.

13. ABSTRACT (Maximum 200 words)

Compact fluorescent (CF) lamps, which are available as an energy-efficient replacement for ordinary incandescent light bulbs, can be used in a variety of fixture types, including existing incandescent, ceiling-mounted, recessed downlights. However, CF lamps are not shaped or sized identically to the incandescent bulbs being replaced; therefore, light distribution will be changed by these retrofits.

Army facilities have numerous incandescent downlight fixtures that are candidates for compact fluorescent retrofits. The information in this study can provide guidance for selecting appropriate retrofits.

This study examined the photometric effects of installing several types of CF lamps in several types of incandescent downlight fixtures. Test results show that, for shallow depth fixtures, several types of CF lamps perform adequately. For deeper fixtures using nonreflective baffle rings, retrofitting with some CF lamps causes light output from the fixture to drop drastically. CF retrofits also caused a wider pattem of light distribution and increased luminance values. Changes in distribution and luminance need to be considered by Army facility managers contemplating CF retrofits.

14. SUBJECT TERMS compact fluorescent retrofits retrofit

lighting equipment

17. SECURITY CLASSIFICATION OF REPORT Unclassified light distribution
5. NUMBER OF PAGES 78

16. PRICE CODE

20. LIMITATION OF ABSTRACT SAR 18. SECURITY CLASSIFICATION OF THIS PAGE Unclassified
19. SECUAITY CLASSIFICATION OF ABSTRACT Unclassified

SAR




\section{Foreword}

This study was conducted for the U.S. Army Center for Public Works (USACPW), and for the Directorate of Military Programs, Headquarters, U.S. Army Corps of Engineers (HQUSACE) under Project 40162784AT45, "Energy and Energy Conservation"; Work Unit FE-XH4, "Lighting Technology Retrofits." The technical monitors were Samuel Baidoo, CECPW-EE, and Robert Billmyre, CEMP-ET-E.

The research was performed by the Utilities Division (UL-U) of the Utilities and Industrial Operations Laboratory (UL), U.S. Army Construction Engineering Research Laboratories (USACERL). The USACERL principal investigator was William $R$. Taylor. Martin J. Savoie is Chief, CECER-UL-U; John T. Bandy is Operations Chief, CECER-UL; and Gary W. Schanche is Chief, CECER-UL.

COL James T. Scott is Commander and Acting Director, USACERL, and Dr. Michael J. O'Connor is Technical Director. 


\section{Contents}

SF 298

1

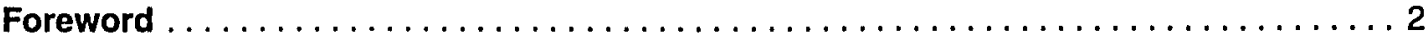

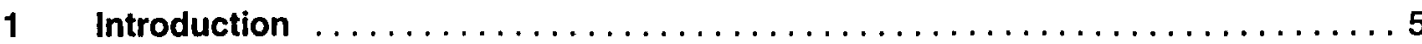

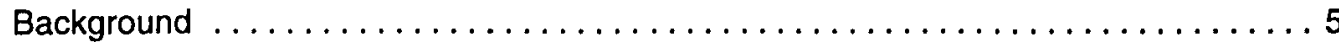

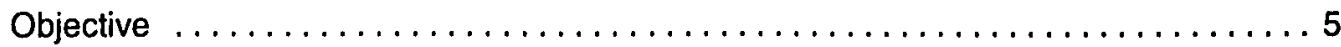

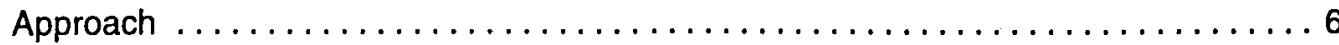

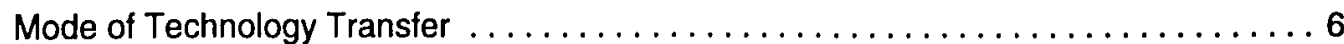

2 General Characteristics of Compact Fluorescent Lamps $\ldots \ldots \ldots \ldots \ldots \ldots \ldots \ldots$

3 CF Retrofit of Incandescent Downlights $\ldots \ldots \ldots \ldots \ldots \ldots \ldots \ldots \ldots \ldots$

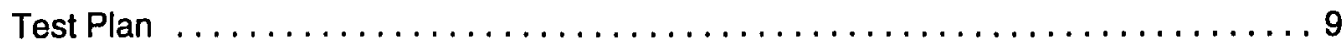

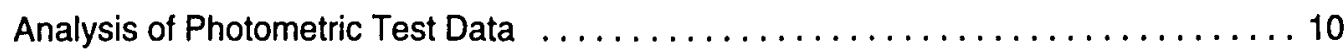

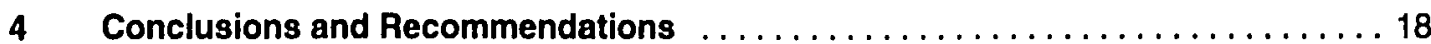

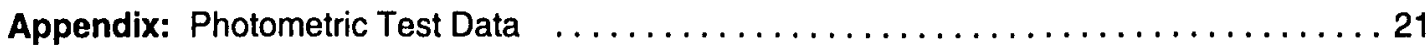

\section{Distribution}





\section{Introduction}

\section{Background}

Compact fluorescent (CF) lamps were introduced in the early 1980s as an energyefficient substitute for the standard incandescent light bulb. CF input power is typically about one-fourth of the input power of the incandescent lamp having approximately the same light output (lumens). Also, CF lamps have a lamp life up to 10 times longer than that of the standard incandescent. Like all gaseous discharge lamps, CFs require a ballast to supply the proper voltage and to control electrical current. CF ballasts are equipped with a screw-in Edison base, which permits CFs to directly replace ordinary incandescent light bulbs. The popularity of CFs due to their energy savings and long life continues to increase despite initial purchase prices that are significantly higher than incandescent lamps.

One popular application of CF lamps is in recessed ceiling downlights, sometimes referred to as "can" downlights. In new construction, downlights designed for CF lamps can be specified. For existing incandescent downlights, a popular and economical retrofit is to replace the existing incandescent lamp with a lower wattage CF lamp. With proper selection, CF lamps can be an excellent retrofit choice. CF lamps are available in a variety of wattages, sizes, shapes, and constructions. Choosing an inappropriate CF lamp for a specific retrofit can lead to deficient lighting conditions related to quantity or quality of light output. Changes in distribution and luminance need to be considered by Army facility managers contemplating CR retrofits. Army facilities have numerous incandescent downlight fixtures that are candidates for compact fluorescent retrofits, and the information in this study can provide guidance for selecting appropriate retrofits.

\section{Objective}

The objective of this study is to test and evaluate the effects on light output of several downlight fixtures produced by retrofits with selected CF lamps. These test results lead to recommendations on selecting or avoiding some specific combinations of downlight fixtures and CF lamps. 


\section{Approach}

Several types of downlight fixtures and common types of CF lamps were selected and purchased for testing. A test plan indicating which lamp/fixture combinations were reasonable for testing was developed. No testing was performed on incompatible lamps and fixtures; for example, when the lamp protruded out of the fixture (although this inappropriate retrofit has occasionally been observed in the field). Photometric testing was performed in a laboratory and results were analyzed.

\section{Mode of Technology Transfer}

The results of these tests will be provided to the U.S. Army Center for Public Works (USACPW), Alexandria, VA for dissemination to Army installations via a Public Works Technical Bulletin (PWTB) or other appropriate publications. 


\section{General Characteristics of Compact Fluorescent Lamps}

This chapter describes some of the basic characteristics and features of compact fluorescent lamp products.

CF lamps are available as modular or integral units. Integral units have lamp and ballast incorporated into one unit; when replacement is required, both lamp and ballast are disposed. Modular units have the advantage that, after the 10,000-hour (typical) lamp life, the lamp can be replaced using the same ballast that might typically have an estimated life of 40,000 to 50,000 hours.

Ballasts may be magnetic or electronic. Generally, magnetic ballasts do not create total harmonic distortion (THD) levels that are considered excessive (since THD values are comparable to standard 4-foot fluorescent lighting, typically in the 10 to 20 percent range). Electronic ballasts have produced electrical current THDs of 100 percent or more. Manufacturers are expected to continue introducing products with lower THDs, but even with the high-percentage THD levels, retrofits using such CF lamps generally have not caused problems in buildings.

Ballasts are also available as normal power factor (NPF) or high power factor (HPF). HPF, by definition, insures a power factor of 90 percent or greater. For retrofit situations, power factor (and THD) are typically not a problem since electrical loading on circuits is likely to be greatly reduced by the retrofit. However, for new construction electrical designers will need to consider power factor and THD to avoid overloading circuits.

CF lamps are available in a variety of correlated color temperatures (CCT) that are measured in kelvins. A common CCT range is 2700 to 3000 , similar to incandescent lamps. CF lamps typically have good color-rendering attributes since most use rare earth phosphors that yield color-rendering index (CRI) values in the 80 s.

CF lamps also come in a variety of shapes, sizes, and wattages. Lamps are configured as twin tubes, double twin tubes, and other shapes, and some lamps offer surrounding reflectors or globe covers. The shape and size of the lamp makes some lamps more or less suitable for use in different types of fixtures. Figure 1 shows the suitability of 
various CF lamps with various fixture types. One type of fixture found in Figure 1 and commonly used in buildings is the ceiling-mounted recessed downlight. Testing and analysis in the following chapters of this report will focus on application of several types of CF lamps in recessed downlights.

\begin{tabular}{|c|c|c|c|c|c|c|c|}
\hline $\bar{A}$ & $\mathbf{P}$ & $L$ & $\mathbf{C}$ & $T$ & 0 & $\mathbf{N}$ & 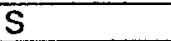 \\
\hline & Downllghts & $\sum_{\text {Surfoce LIghts }}$ & $\sum_{\text {Pandant Fixturo: }}$ & 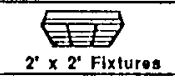 & 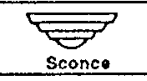 & $\begin{array}{l}\text { EXXX] } \\
\text { Exit/1/lop }\end{array}$ & $\begin{array}{l}\text { (1) } \\
\text { Floodllghts }\end{array}$ \\
\hline$\overbrace{\text { Incendoscont }}^{\infty}$ & + & + & + & - & + & + & + \\
\hline The Tw/n-Tube & + & + & - & - & + & + & + \\
\hline$\underbrace{}_{T-4 \text { Qund-Tube }}$ & + & + & + & - & + & - & $=$ \\
\hline$\bigcup_{\text {Intogral Ballest Lemp }}^{\coprod_{0}}$ & + & - & ++ & - & - & - & - \\
\hline$\sum_{\text {clrelline }}$ & - & + & + & - & + & - & - \\
\hline Roflloclor Unit & ++ & + & + & - & - & - & + \\
\hline & & & $\begin{array}{l}\text { Suparlor lamp chocke } \\
\text { Sullablo lamp cholce } \\
\text { unsulablo lamp cholce }\end{array}$ & & & & \\
\hline
\end{tabular}

Figure 1. Applications of CF lamps in various fixtures. 


\section{CF Retrofit of Incandescent Downlights}

Both incandescent fixtures and compact fluorescent lamps come in a variety of sizes and shapes, which creates the possibility of numerous retrofit combinations of fixtures and lamps (Figure 1 shows which CF lamp types work best with various fixture types). One of the most popular CF retrofits is in incandescent downlight ("can") fixtures. This chapter examines the effects of various CF lamp types in incandescent fixtures.

\section{Test Plan}

Several incandescent downlight fixtures were selected to be representative of commonly found fixtures in the field. The fixtures selected were:

- Incandescent downlight, shallow ( $7 \frac{3}{8}$ in.) can, about 6 in. diameter, short reflector

- Incandescent downlight, shallow ( $7 \frac{3 / 8}{\mathrm{in}}$.) can, about 6 in. diameter, full reflector

- Incandescent downlight, deep (11 in.) can, about 6 in. diameter, full reflector

- Incandescent downlight, deep (11 in.) can, about 6 in. diameter, no reflector, black baffle

- CF downlight, shallow can, about 6 in. diameter, partial reflector, verticallamp

- CF downlight, shallow can, about 6 in. diameter, partial reflector, horizontal lamp.

Several types of CF lamps were selected to be representative of lamps that might commonly be selected for retrofits. The CF lamp types selected were:

- 15-watt integral unit with opal globe (bullet shaped)

- 11-watt double twin tube integral unit

- 13-watt double twin tube modular unit, adapter (ballast) selected by test lab

- 13-watt double twin tube modular unit with reflector and lens, adapter from reflector manufacturer

- 15-watt double twin tube modular unit with open reflector. 
For comparison with the CF lamps, a 60-watt A-19 incandescent lamp and a 150-watt R40 spot incandescent lamp were tested in the incandescent downlights. Also for comparison, two CF fixtures with a 13-watt double twin tube CF lamp were tested.

Figure 2 shows a matrix of lamp types and fixture types and indicates which lamp and fixture combinations were tested. Test numbers (e.g., ITL40777) correspond to the photometric reports in the Appendix. Where lamp/fixture combinations indicate no test was performed, the primary reasons for not testing were (1) the fixture reflector was too narrow at the base to allow the CF lamp to reach the socket, or (2) the CF lamp was too long, causing the lamp to protrude out of the fixture.

\section{Analysis of Photometric Test Data}

The photometric test data (shown in the Appendix) is presented in Figures 3 through 7 to permit a comparison of data from identical tests performed on various lamp/ fixture combinations.

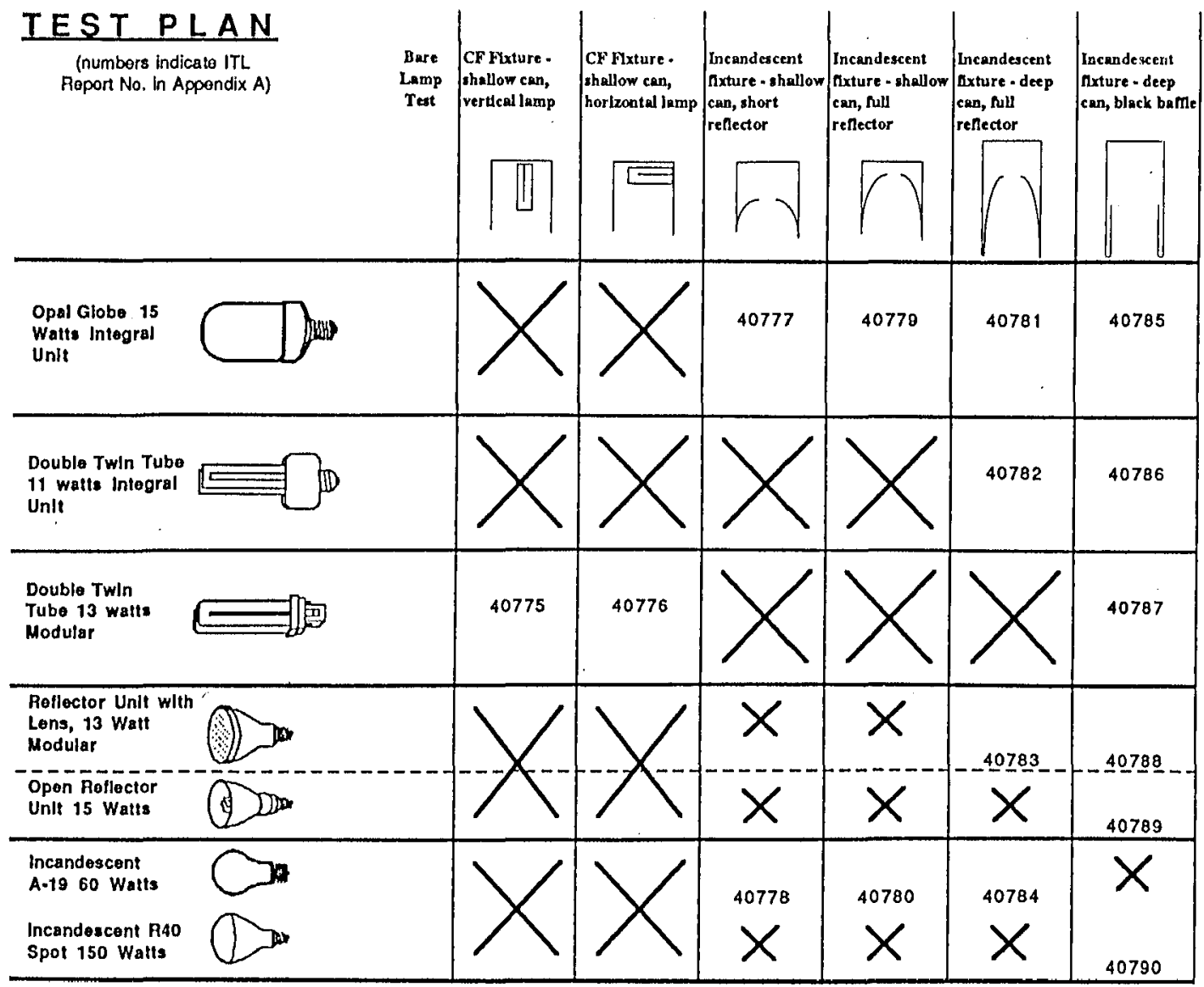

$>$ indicates NO TEST performed (all remaining squares tested) 


\section{Total Luminaire Efficiency}

Figure 3 shows luminaire efficiencies from the test data. The CF fixture with the horizontally mounted lamp has a luminaire efficiency of 66 percent compared to 44 percent for the vertically mounted lamp. Incandescent fixture efficiencies ranged from 56 to 65 percent. Efficiency data for the shallow can fixtures and the deep can fixture with reflector ranged from 70 to 80 percent except for the enclosed reflector lamp in the deep can fixture, which had a 44 percent efficiency. Several of the CF lamps were not tested in these fixtures (for reasons stated before). The deep can fixture with black baffle showed very low efficiencies with all but one of the CF lamps, with efficiencies ranging from 9 to 28 percent except for the open reflector lamp, which raised luminaire efficiency to 72 percent.

\section{Zonal Lumen Summary and Bare Lamp Lumen Outputs}

Figure 4 shows lumen output for the lamp/fixture test combinations, calculated by multiplying the luminaire efficiency by the measured lamp lumen output. (Note that total lumens in the zonal lumen summary of the photometric data is calibrated for the lamps' nominal rated lumens, not measured bare lamp lumens.) Photometric testing of the bare lamps showed total lumen output to be lower than lamp rated lumens for all lamps but one.

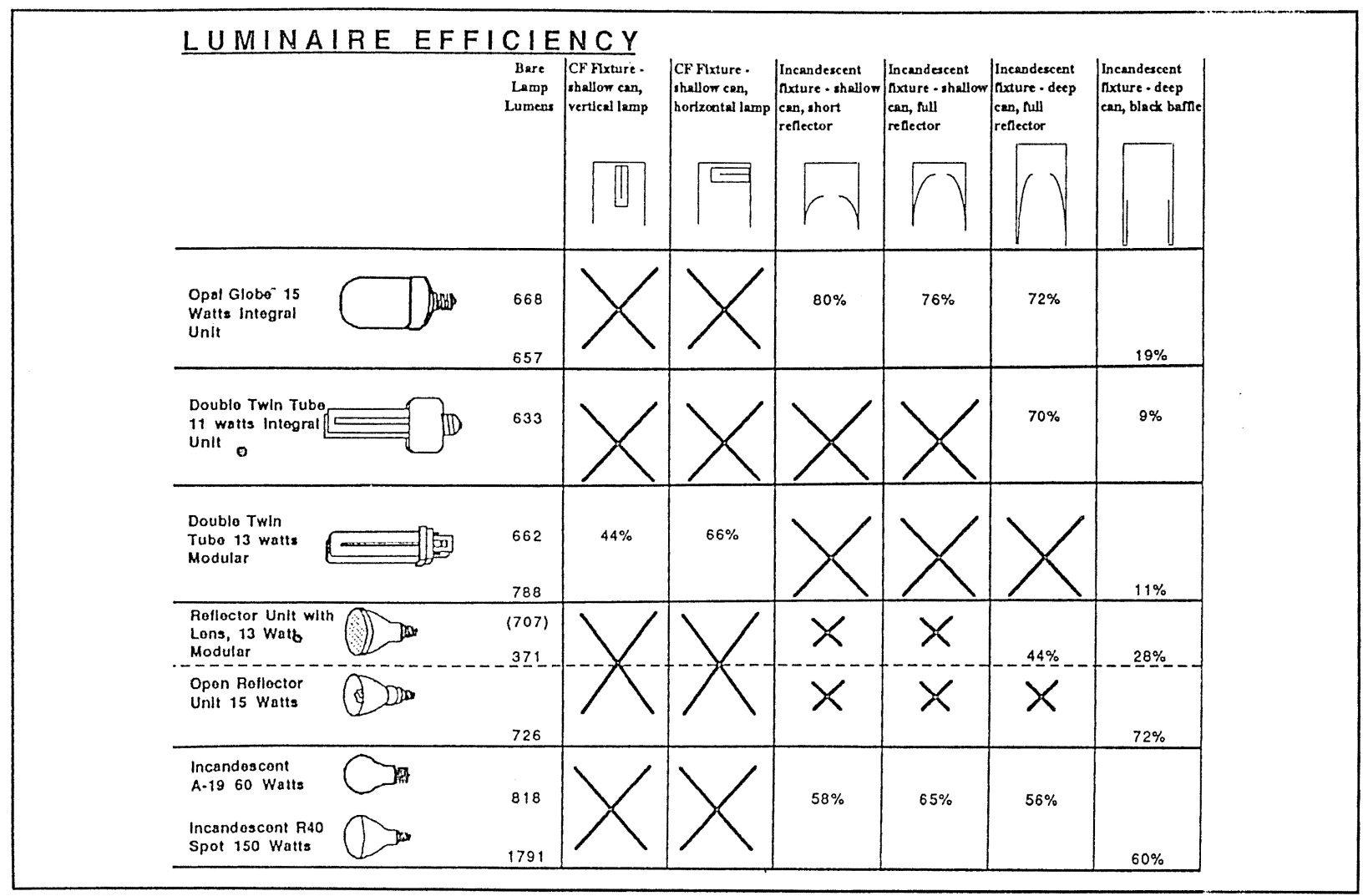

Figure 3. Luminaire efficiencies. 


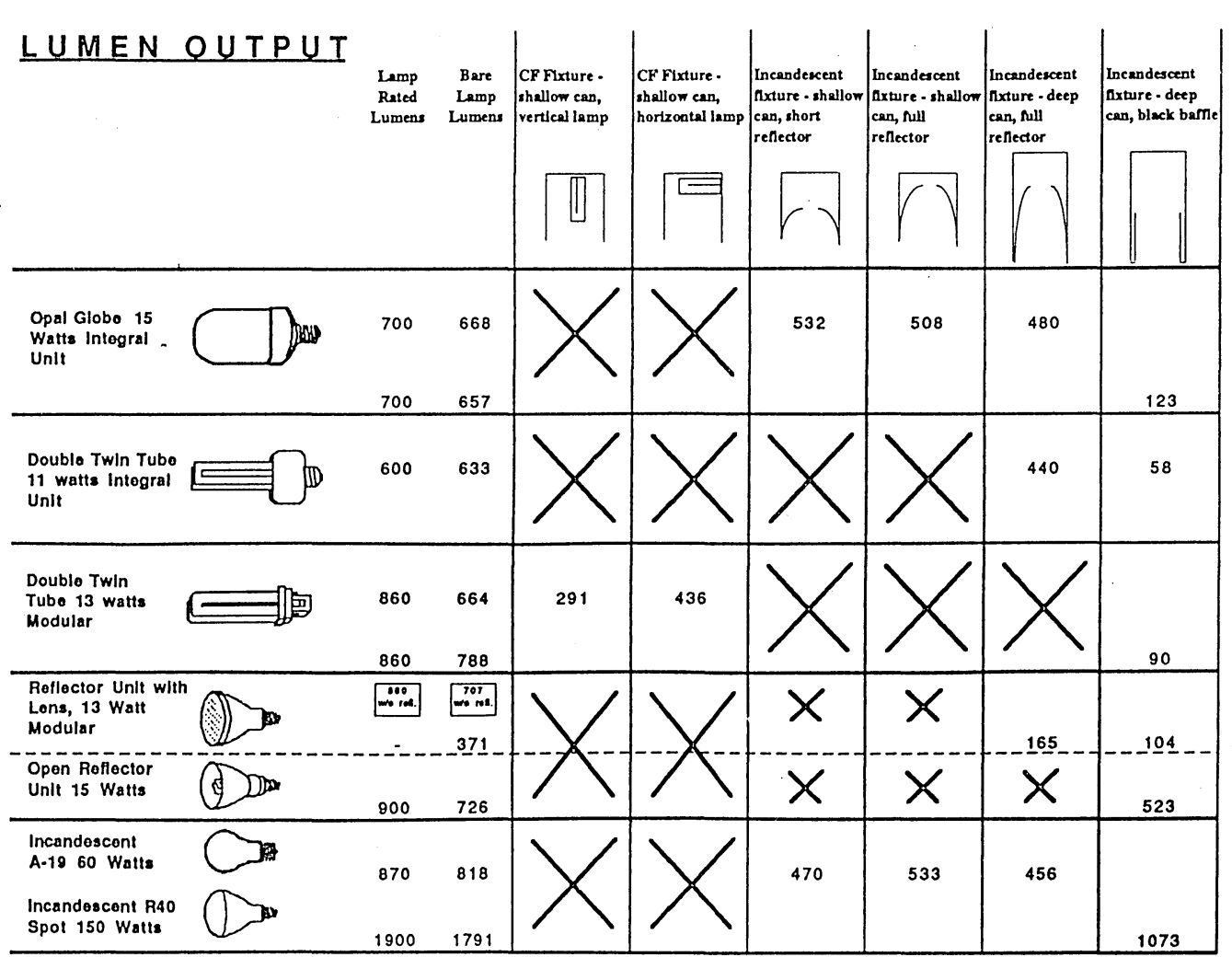

Figure 4. Lumen output for lamp/fixture test combinations.

Lumen output for the following lamp/fixture combinations were comparable to the 60watt incandescent lamp: (1) the opal globe CF lamp with the 3 reflector fixtures; (2) the double twin tube 11-watt unit with the deep can, full reflector fixture; (3) the double twin tube 13-watt unit in the horizontally mounted CF fixture; and (4) the open reflector 13-watt unit in the deep can, black baffle fixture.

The 150-watt R40 spot lamp was tested in the deep can black baffle fixture to represent a lamp that might be used in such a fixture in the field, given the maximum wattage stamped on the fixture was 150 watts. As expected, lumen output of the 150watt lamp was significantly greater than the other lamp/fixture combinations, approximately double the lumen output of the 60-watt incandescent in the reflector fixtures and other comparable combinations.

\section{Input Power and Efficacy}

Figure 5 shows input watts for the lamps and the calculated efficacy (lumens/watt) for both the bare lamps and the lamp/fixture combinations. (The lumen values from Figure 4 were used to calculate efficacy.) 


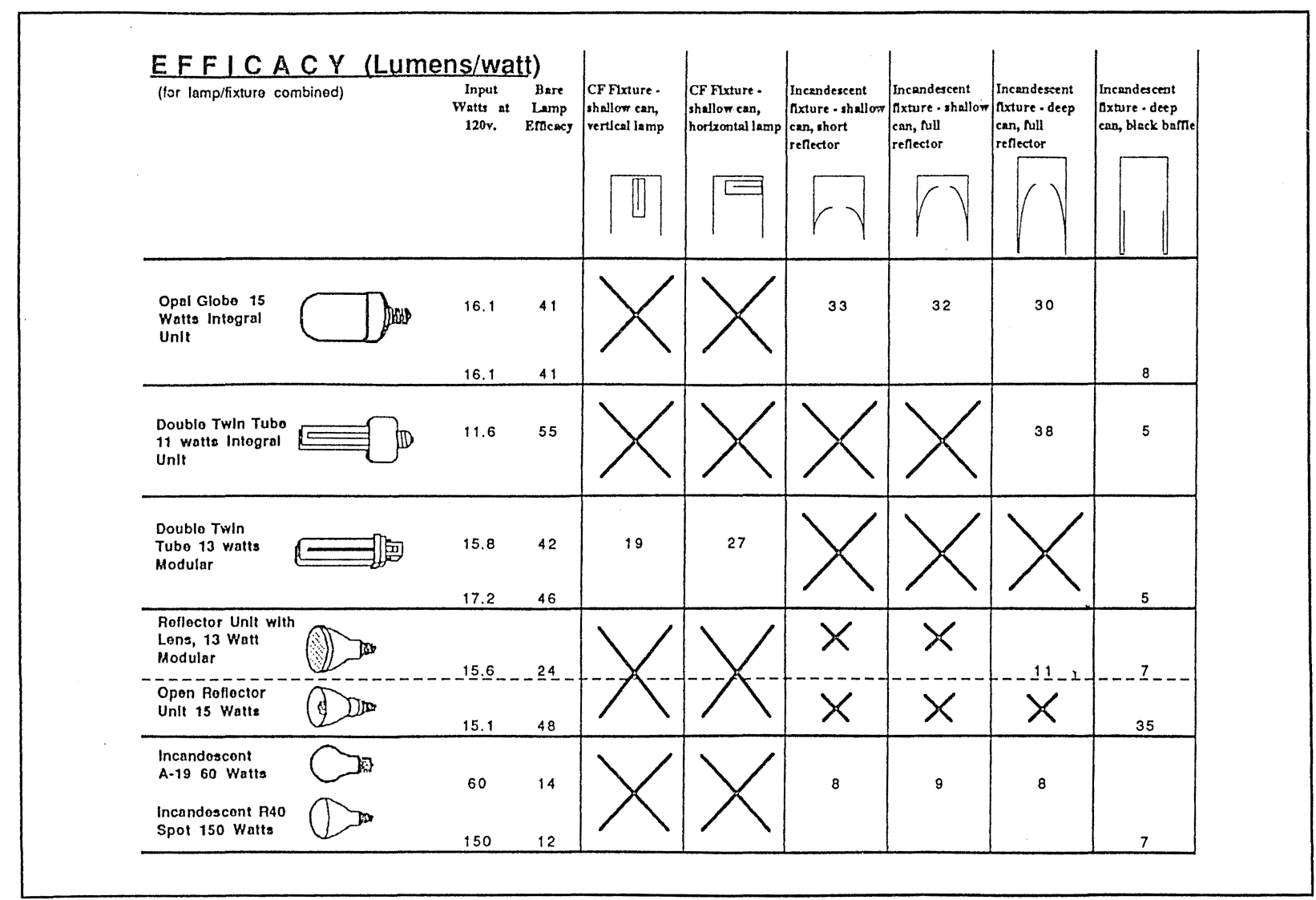

Figure 5. Efficacy (lumens/watt) for lamp/fixture combinations.

Efficacies for the two incandescent lamps were 12 and 14 lumens per watt $(\mathrm{lm} / \mathrm{W})$ for the bare lamps and ranged from 7 to $9 \mathrm{~lm} / \mathrm{W}$ for the combined lamp and fixture. CF bare lamp efficacies ranged from 24 to $55 \mathrm{~lm} / \mathrm{W}$. CF lamp and fixture combinations produced efficacies of 19 to $38 \mathrm{~lm} / \mathrm{W}$ for reasonable retrofit combinations and efficacies of 5 to $7 \mathrm{~lm} / \mathrm{W}$ for less desirable retrofits.

\section{Candela Distribution Curves}

Figure 6 shows candela distribution curves for the lamp/fixture test combinations. The curves are presented in reduced size which, although it is difficult to read the scales, will permit side-by-side comparison of the light distribution patterns. The Appendix contains more legible curves.

Candela distribution for the incandescent lamps in various fixtures all exhibited a relatively narrow beam spread. The $\mathrm{CF}$ reflector lamps had a slightly less narrow beam, and the opal globe unit with a length that protruded nearly to the end of the fixture exhibited a wide beam spread. The 11-watt CF required several adapters to reach the socket and, therefore, also reached nearly to the end of the fixture, producing 


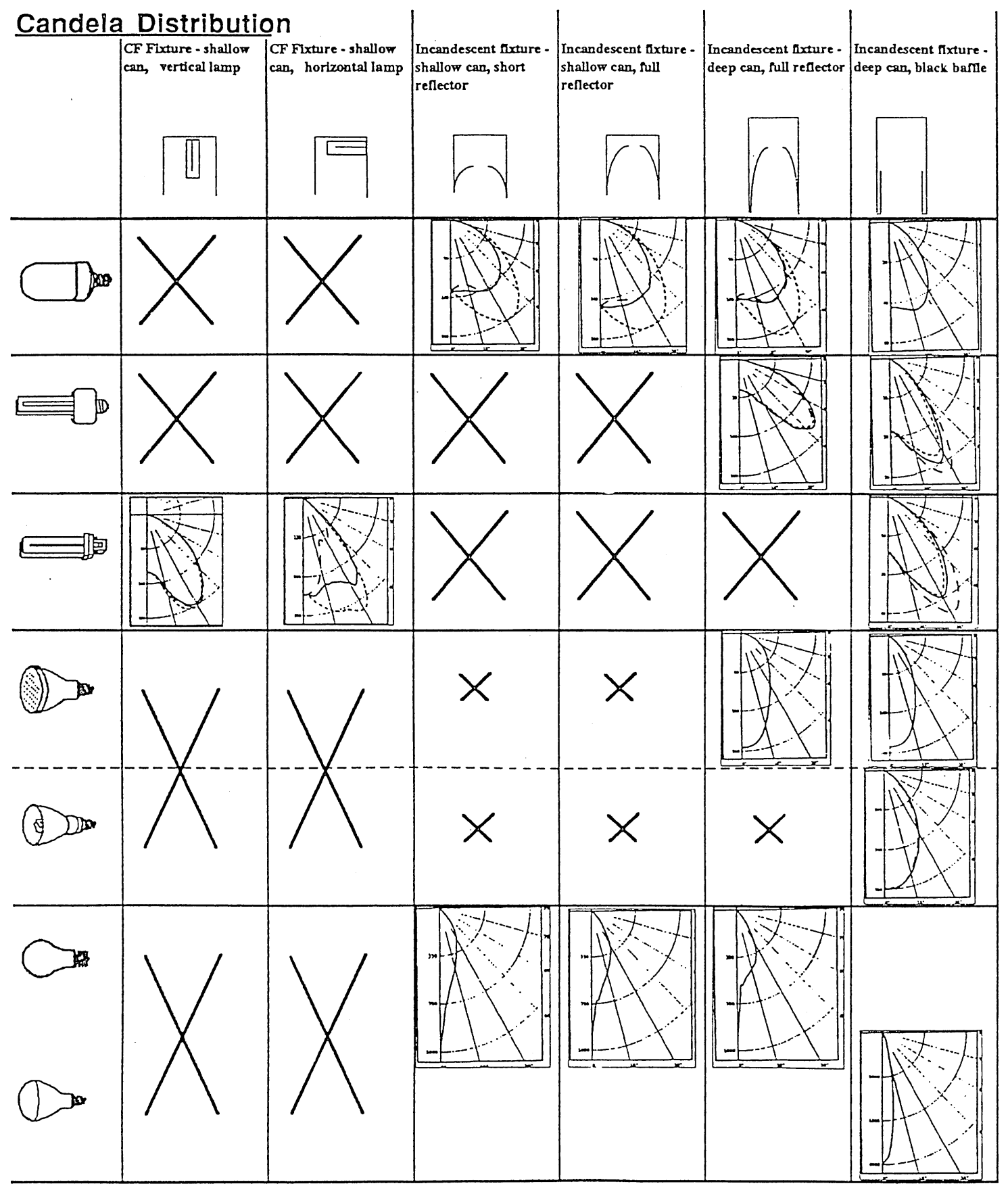

Figure 6. Candela distribution curves for lamp/fixture test combinations. 
a wide beam spread. The CF fixtures exhibited a medium beam spread. Not surprisingly, the horizontally mounted CF produced a less symmetric beam than the vertically mounted $\mathrm{CF}$.

\section{Luminance Data}

Luminance data provides an indication of the "brightness" of the fixture opening when viewed from various angles. For the fixtures tested, the fixture opening is approximately a 6 -inch diameter when viewed directly beneath the fixture. Luminance data shown in Figure 7 indicates the average and maximum luminance of the fixture opening, and a ratio of maximum to average luminance is calculated.

As expected, the incandescent lamps with narrow beam patterns, probably indicative of a light source recessed into the fixture, had very low luminance values at medium and high cutoff angles. Luminance values for the deep can black baffle fixture were very low.

The opal globe lamp produced slightly higher luminance values through a broader range of viewing angles than the other CF lamps. However, the maximum to average ratios were very low, probably due to the soft light appearance of the opal globe. 


\begin{tabular}{|c|c|c|c|c|c|c|}
\hline Luminar & $\begin{array}{l}\text { nce Data } \\
\begin{array}{l}\text { CF Fixture - shallow } \\
\text { can, vertical lamp }\end{array}\end{array}$ & $\begin{array}{l}\text { CF Fixture - shallow } \\
\text { can, horizontal lamp }\end{array}$ & $\begin{array}{l}\text { Incandescent fixture - } \\
\text { shallow can, short } \\
\text { reflector }\end{array}$ & $\begin{array}{l}\text { Incandescent fixture - } \\
\text { shallow can, full } \\
\text { reflector }\end{array}$ & $\begin{array}{l}\text { Incandescent fixture - } \\
\text { deep can, full reflector }\end{array}$ & $\begin{array}{l}\text { Incandescent fixture - } \\
\text { deep can, black baffle }\end{array}$ \\
\hline & & & 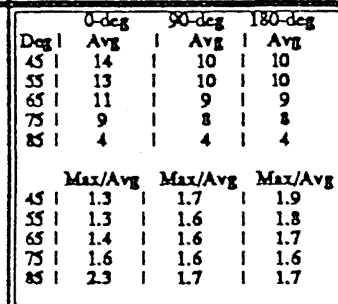 & 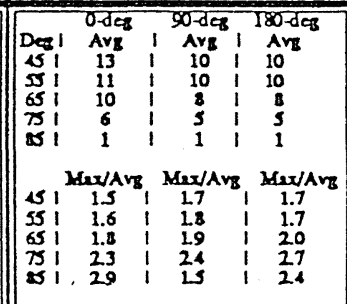 & 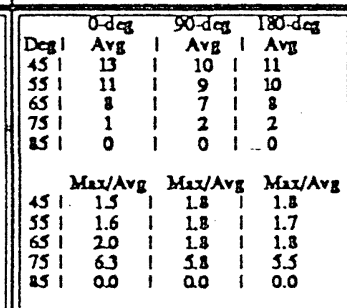 & 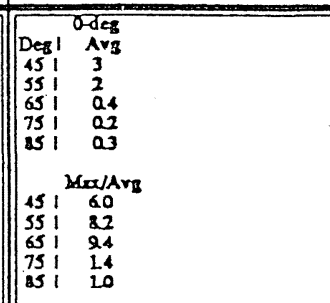 \\
\hline & & & & & 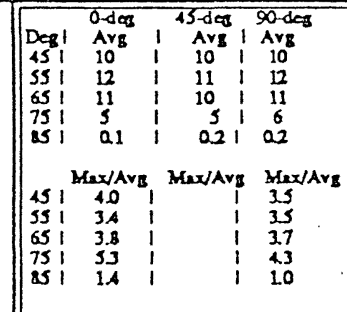 & 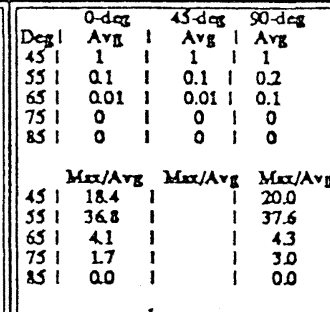 \\
\hline 一思 & 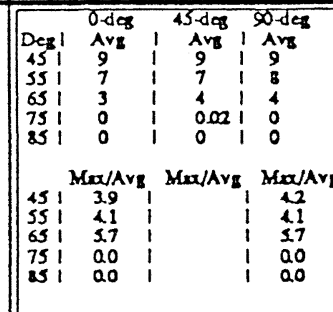 & 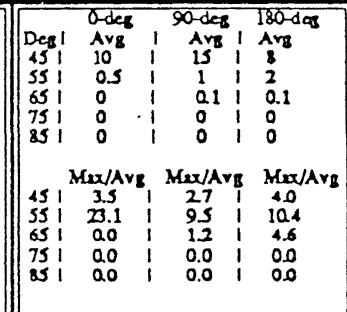 & & & & 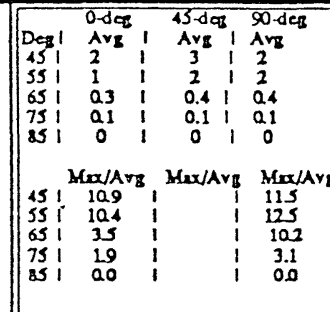 \\
\hline
\end{tabular}




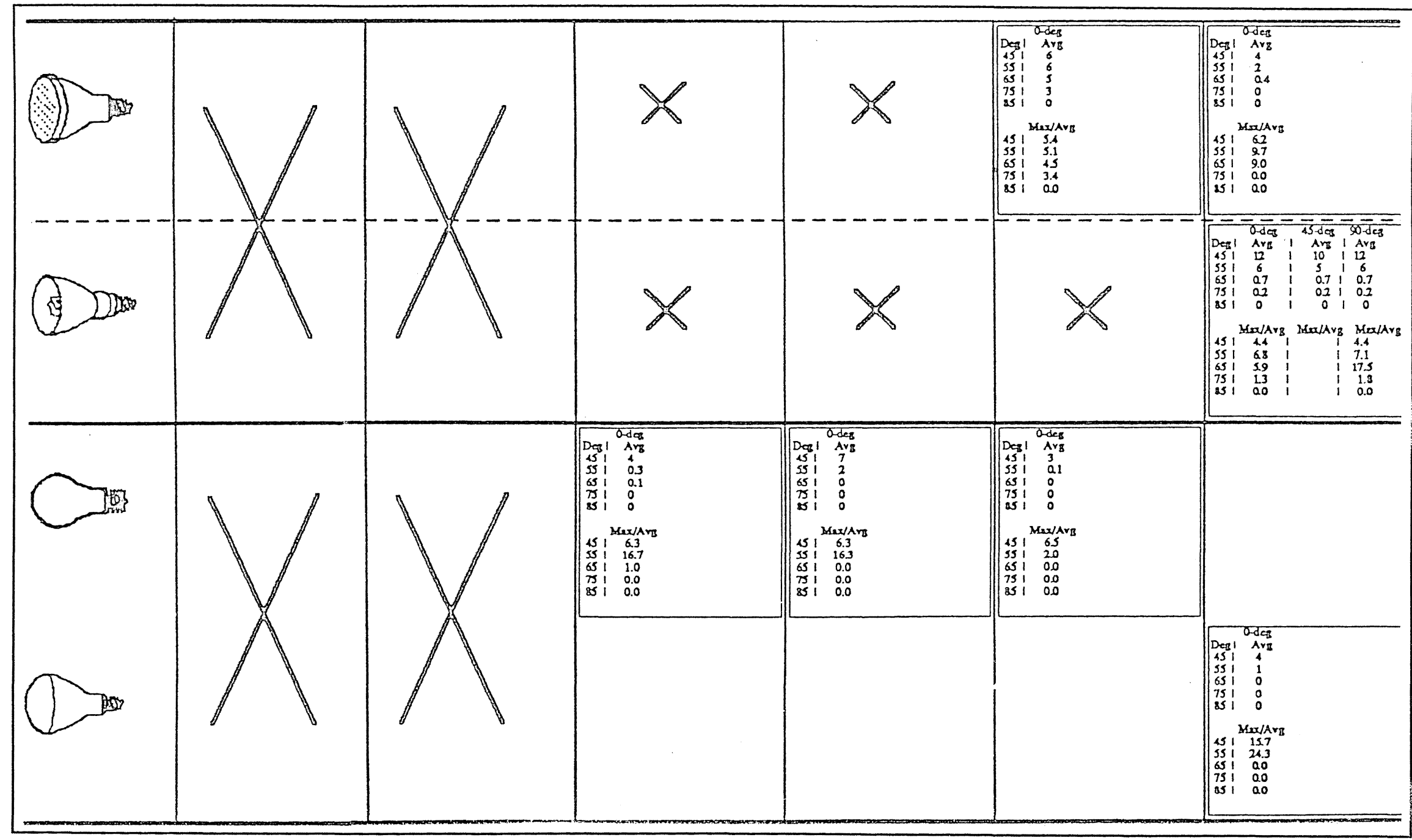

Figure 7. (Cont'd) 


\section{Conclusions and Recommendations}

Of the two CF fixtures tested, the horizontally mounted lamp configuration showed a significantly higher fixture efficiency than the vertically mounted lamp. It is recommended that designers be aware of this and, where manufacturers' data confirms this to be the case, specify horizontally mounted lamps in CF fixtures.

When retrofitting incandescent downlights that are constructed as a deep (about 11 in.) can with a black baffle ring, selection of a suitable CF lamp is critical. From the limited testing being reported here, the only CF lamp that was capable of directing light out of the fixture with reasonable efficiency (72 percent) was the open-ended reflector CF lamp unit. Other CF lamps caused fixture efficiency to drop to a range of 9 to 28 percent.

Several of the CF lamps that were retrofitted into incandescent downlights for these tests produced fixture efficiencies that were as high as the efficiency of the CF fixture manufactured for use with a CF lamp.

All but one of the CF lamps tested produced lower bare lamp lumens than the nominal lumen rating of the lamp. While the sample size of this testing was very small, these results are consistent with observations in other testing. ${ }^{*}$ Lumen output of several $\mathrm{CF}$ lamps in the various fixtures confirmed the general rule-of-thumb that CF lamps consuming one-fourth the watts of the incandescent being replaced will produce approximately the same amount of light; this assumes a proper retrofit lamp selection for the fixture. Inappropriate CF lamp and fixture combinations showed overall efficacies no better than properly matched incandescent lamps and fixtures.

Candela distribution indicates that fixtures with the CF lamps create a wider spread in the distribution pattern. Even when total lumen output is approximately equal, the incandescents provide more intense light directly beneath the fixture ( 0 to 30 degrees) while the CF lamps provide more light than incandescents at 50 to 60 degrees. For general area lighting applications, this wider distribution from $C F$ lamps may not be a problem. In applications where downlights are lighting a specific area (walls, objects, and so on), the wider distribution could be undesirable. In such cases it is

* Lighting Research Center, "Screwbase Compact Fluorescent Lamp Products," Speci.ier Reports, vol 1, No. 6 (April 1993). 
recommended that a trial installation of the proposed retrofit be used to determine the appropriateness of the selected CF lamp.

In addition to affecting the candela distribution, the geometry and placement of retrofit CF lamps will affect the luminance of the light source, with excessive luminance causing discomfort glare. The test data indicate luminance values for many of the lamp/fixture combinations (including the incandescents) exceed the Illuminating Engineering Society of North America (IESNA) values, ${ }^{*}$ which indicates that discomfort glare is a problem. The opal globe CF units had the most consistently high luminance values, but they also had some of the lowest maximum to average luminance ratios. The diffusing action of the opal globe reduces the luminance difference between the brightest spot and the surrounding area of the fixture. While luminance data from these tests do not show that discomfort glare will be a problem for various field applications, the generally higher luminance values for CF lamps compared to the incandescent lamps is a warning that discomfort glare warrants consideration when evaluating retrofits.

The conclusions from this testing are summarized in Figure 8, which shows lamp/fixture combinations recommended or not recommended for retrofits. It is assumed that even for the recommended retrofits, the user will need to evaluate the potential change in candela distribution and luminance as discussed previously. 


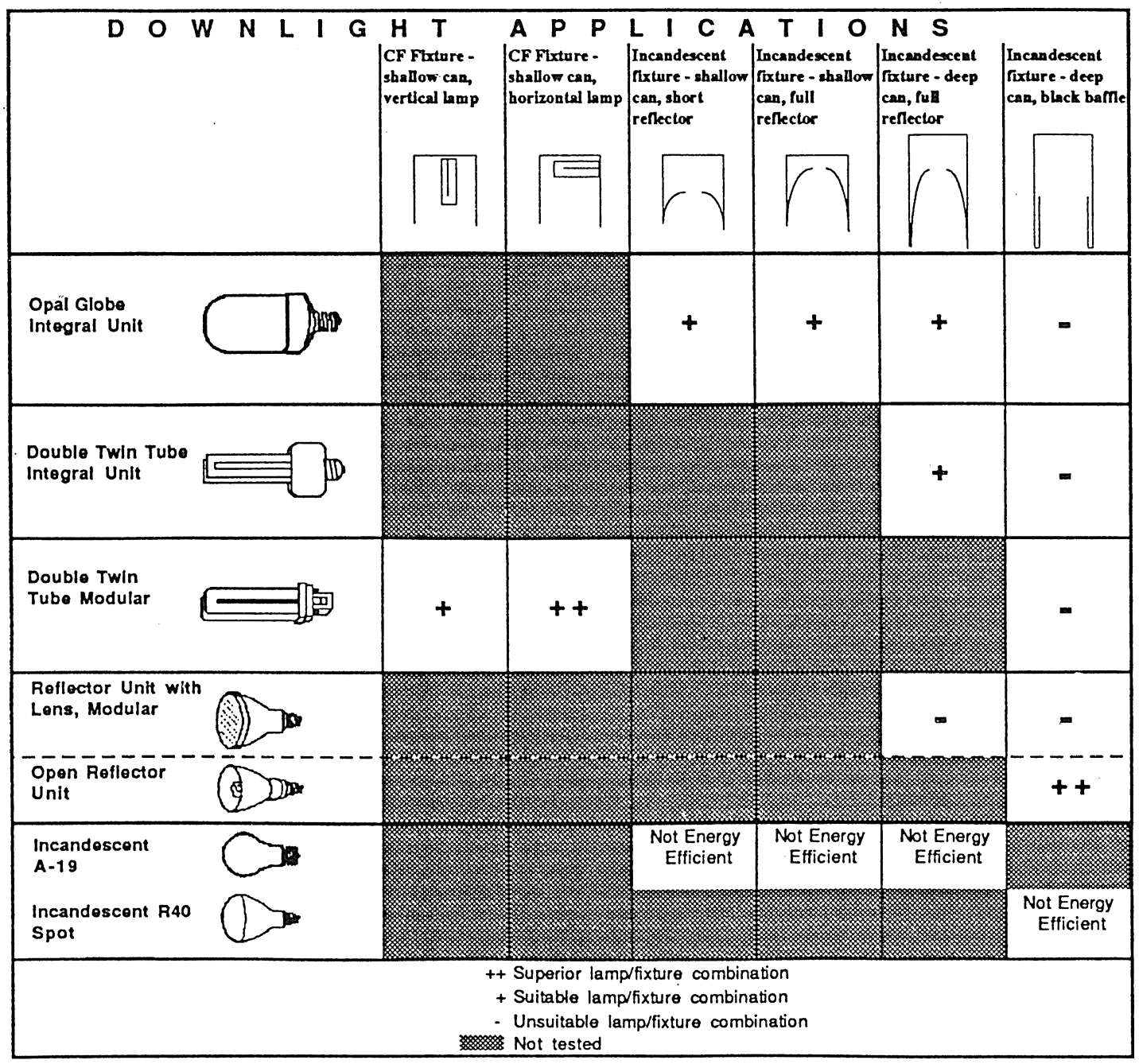

Figure 8. Summary of testing of lamp/fixture combinations. 


\section{Appendix: Photometric Test Data}




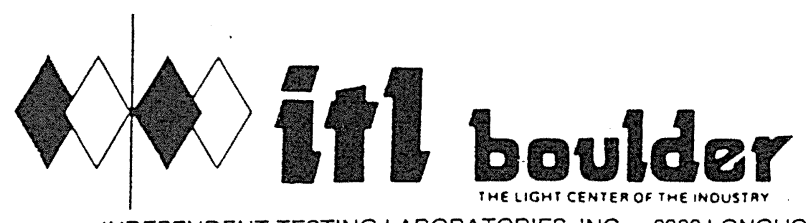

INDEPENDENT TESTING LABORATORIES, INC. • 3386 LONGHORN ROAD, BOULDER. COLORADO 80302 • PHONE (303) 442-19

REPORT NUMBERS: ITL40775-ITL40790

PREPARED FOR: U.S. ARMY CORPS OF ENGINEERS

DATE: $8-30-1993$

\section{ABSOLUTE LUMEN OUTPUTS}

AIl lamps were seasoned according to IES LM-54 prior to testing. Lumen output of each lamp was measured prior to photometric testing. The lamps were mounted on the goniophotometer and twelve planes of data were recorded at 2.5 degree vertical increments. The planes were averaged and lumen output was calculated using zonal constants from the candela data. The candela calibration is traceable to tie National Institute of Standards and Technology (formerly the National Bureau of Standards). Testing was performed in a $25+1-1$ degree celsius free air ambient. Lumen output is for the particular sample when operated on the ballast described in the report. Due to retesting, the lumen output of the FLB15/TL was measured more than once. The Lumatech RAS-130 was measured bare and with the reflector installed, both values are shown. Since the lumen rating is for the bare lamp, the bare lamp lumen output was used for calculating luminaire efficiency. All photometric reports are issued as standard relative photometry, all data is reported as if the lamps produced rated lumens.

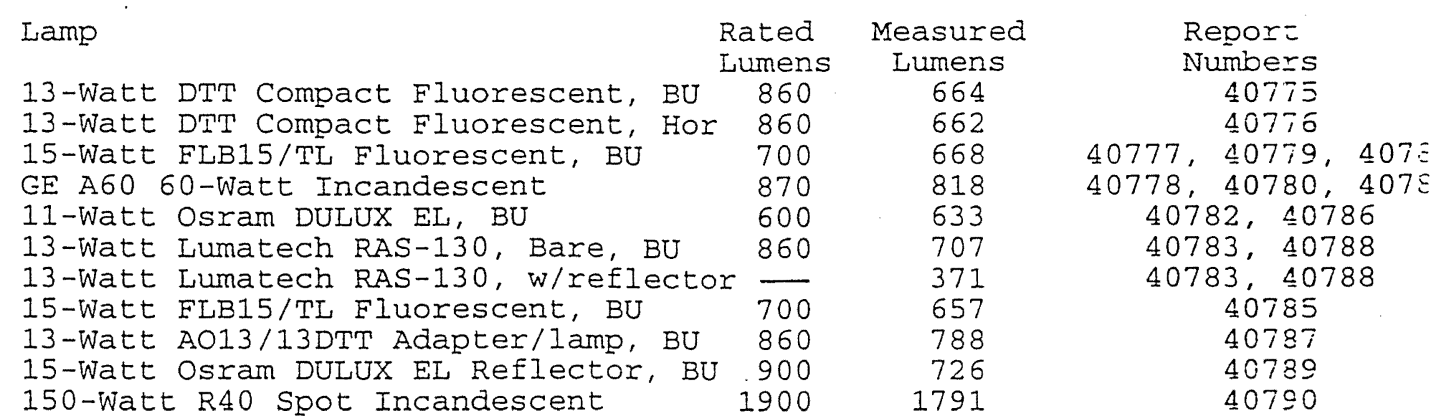

\section{MAXIMUM LUMINANCE MEASUREMENTS}

Maximum Iuminance was measured using a Tektronix J6523 1 Degree Iuminance probe instead of ITL's standard Maximum Brightness meter due to the smal: luminous area of these units. All values were adjusted using the ratio $c$ the rated lumens versus the measured lumens and reported at rated lamp lumens (standard relative photometry procedure).

NOTE: Test ITL40784 required the addition of one adapter in order for the lamp to screw into the socket.

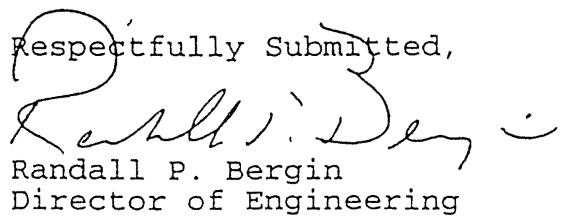

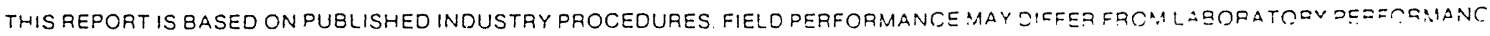




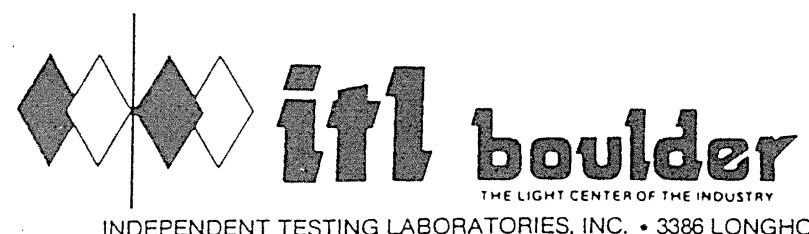

INDEPENDENT TESTING LABORATORIES, INC. • 3386 LONGHORN ROAD, BOULDER, COLORADO 80302 • PHONE (303) 442-1255 REPORT NUMBER: ITL40775

PREPARED FOR: U.S. ARMY CORPS OF ENGINEERS

CATALOG NUMBER: SHALLOW CAN, VERTICAL LAMP

LUMINAIRE: FABRICATED CYLINDRICAL METAL HOUSING WITH WHITE PAINTED INTERIOR, SPECULAR ALUMINUM LOWER CONE REFLECTOR, WHITE PAINTEI METAL TRIM RING, OPEN BOTTOM.

LAMP: 13-WATT DOUBLE TWIN TUBE COMPACT FLUORESCENT, RATED 860 LUMENS, VERTICAL BASE-UP POSITION.

BALLAST: ROBERTSON HP-S1

MOUNTING: RECESSED

TOTAL INPUT WATTS $=15.6$ AT 120.0 VOLTS

CANDELA DISTRIBUTION

$\begin{array}{rrrrrr} & 0.0 & 22.5 & 45.0 & 67.5 & 90.0 \\ 0 & 83 & 83 & 83 & 83 & 83 \\ 5 & 85 & 85 & 85 & 85 & 85 \\ 15 & 120 & 120 & 116 & 113 & 110 \\ 25 & 150 & 152 & 146 & 147 & 143 \\ 35 & 141 & 146 & 145 & 147 & 142 \\ 45 & 108 & 111 & 111 & 113 & 113 \\ 55 & 72 & 72 & 72 & 74 & 75 \\ 65 & 25 & 25 & 26 & 28 & 28 \\ 75 & 0 & 0 & 0 & 0 & 0 \\ 85 & 0 & 0 & 0 & 0 & 0 \\ 90 & 0 & 0 & 0 & 0 & 0\end{array}$

ZONAL LUMEN SUMMARY

ZONE LUMENS

$\begin{array}{rr}0-30 & 110 \\ 0-40 & 200 \\ 0-60 & 350 \\ 0-90 & 377 \\ 90-180 & 0 \\ 0-180 & 377\end{array}$

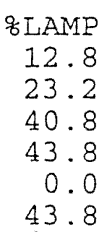

TOTAL LUMINAIRE EFFICIENCY = CIE TYPE - DIRECT
PLANE
$0-D E G \quad 90-D E G$
SPACING CRITERIA : 1.9 2.0
LUMINOUS DIAMETER: $\quad 5.750$

LUMINANCE DATA IN CANDELA/SO M

ANGLE AVERAGE AVERAGE AVERAGE

IN DEG 0-DEG 45-DEG 90-DEG

459129.9391 .9492$.

$55 \quad 7479.7489 .7812$

653473.3656 .3981$.

7500.23 .0$.

8500.

ANGLE MAXIMUM MAXIMUM MAX/AVG MAX/AVG

IN DEG O-DEG 90-DEG 0-DEG 90-DEG

$\begin{array}{rrrrr}45 & 35151 . & 40167 . & 3.9 & 4.2 \\ 55 & 30358 . & 32400 . & 4.1 & 4.1 \\ 65 & 19839 . & 22724 . & 5.7 & 5.7 \\ 75 & 352 . & 138 . & 0.0 & 0.0\end{array}$

$43.8 \%$
FAX (303) 449-5274 DATE: 7-26-1993

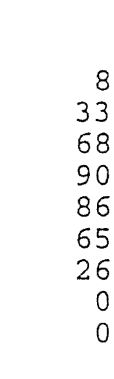

$$
\begin{array}{r}
\text { \&EIXT } \\
29.1 \\
53.0 \\
93.0 \\
100.0 \\
0.0 \\
100.0
\end{array}
$$

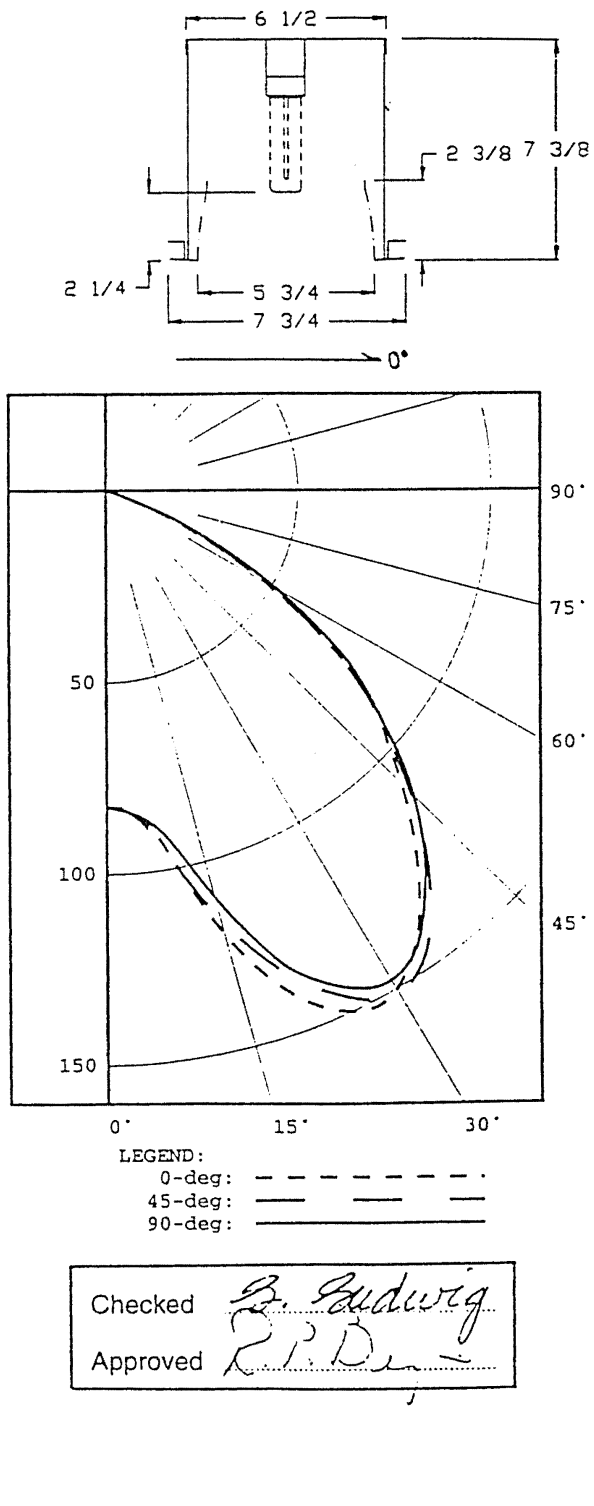

THIS REPORT IS BASED ON PUBLISHED INDUSTRY PROCEDURES. FIELD PERFORMANCE MAY DIFFER FROM LABORATORY PERFORMANCE 


\section{itl boulder}

$\operatorname{FAX},(303) 449-5274$ INDEPENDENT TESTING LABORATORIES, INC. • 3386 LONGHORN ROAD, BOULDER. COLORADO 80302 • PHONE (303) 442-1255

REPORT NUMBER: ITL40775

PREPARED FOR: U.S. ARMY CORPS OF ENGINEERS

DATE: $7-26-1993$

CANDELA DISTRIBUTION

\begin{tabular}{|c|c|c|c|c|c|c|c|}
\hline & 0.0 & 22.5 & 45.0 & 67.5 & 90.0 & ZONAL LUMEN & SUMMARY \\
\hline 0.0 & 83 & 83 & 83 & 83 & 83 & $0-$ & 2 . \\
\hline 2.5 & 83 & 83 & 83 & 83 & 83 & $5-10$ & 6. \\
\hline 5.0 & 85 & 85 & 85 & 85 & 85 & $10-15$ & 13. \\
\hline 7.5 & 89 & 90 & 90 & 88 & 87 & $15-20$ & 21. \\
\hline 10.0 & 97 & 98 & 98 & 94 & 92 & $20-25$ & 30. \\
\hline 12.5 & 108 & 109 & 106 & 103 & 100 & $25-30$ & 38. \\
\hline 15.0 & 120 & 120 & 116 & 113 & 110 & $30-35$ & 44. \\
\hline 17.5 & 130 & 131 & 125 & 124 & 120 & $35-40$ & 46. \\
\hline 20.0 & 139 & 140 & 133 & 134 & 130 & $40-45$ & 45. \\
\hline 22.5 & 146 & 147 & 140 & 141 & 137 & $45-50$ & 41 \\
\hline 25.0 & 150 & 152 & 146 & 147 & 143 & $50-55$ & 36. \\
\hline 27.5 & 152 & 155 & 150 & 152 & 146 & $55-60$ & 28 . \\
\hline 30.0 & 150 & 155 & 151 & 153 & 147 & $60-65$ & 18. \\
\hline 32.5 & 146 & 152 & 149 & 152 & 146 & $65-70$ & 8. \\
\hline 35.0 & 141 & 146 & 145 & 147 & 142 & $70-75$ & 0. \\
\hline 37.5 & 133 & 139 & 138 & 140 & 135 & $75-80$ & 0. \\
\hline 40.0 & 125 & 130 & 130 & 132 & 129 & $80-85$ & 0. \\
\hline 42.5 & 117 & 121 & 121 & 123 & 121 & $85-90$ & 0 . \\
\hline 45.0 & 108 & 111 & 111 & 113 & 113 & & \\
\hline 47.5 & 100 & 102 & 102 & 104 & 103 & & \\
\hline 50.0 & 93 & 93 & 93 & 94 & 93 & & \\
\hline 52.5 & 84 & 84 & 83 & 85 & 85 & & \\
\hline 55.0 & 72 & 72 & 72 & 74 & 75 & & \\
\hline 57.5 & 59 & 60 & 60 & 62 & 63 & & \\
\hline 60.0 & 47 & 48 & 49 & 50 & 51 & & \\
\hline 62.5 & 36 & 36 & 37 & 39 & 40 & & \\
\hline 65.0 & 25 & 25 & 26 & 28 & 28 & & \\
\hline 67.5 & 14 & 14 & 15 & 17 & 18 & & \\
\hline 70.0 & 2 & 3 & 4 & 4 & 5 & & \\
\hline 72.5 & 0 & 0 & 0 & 0 & 0 & & \\
\hline 75.0 & 0 & 0 & 0 & 0 & 0 & & \\
\hline 77.5 & 0 & 0 & 0 & 0 & 0 & & \\
\hline 80.0 & 0 & 0 & 0 & 0 & 0 & & \\
\hline 82.5 & 0 & 0 & 0 & 0 & 0 & & \\
\hline 85.0 & 0 & 0 & 0 & 0 & 0 & & \\
\hline 87.5 & 0 & 0 & 0 & 0 & 0 & & \\
\hline 90.0 & 0 & 0 & 0 & 0 & 0 & & \\
\hline
\end{tabular}




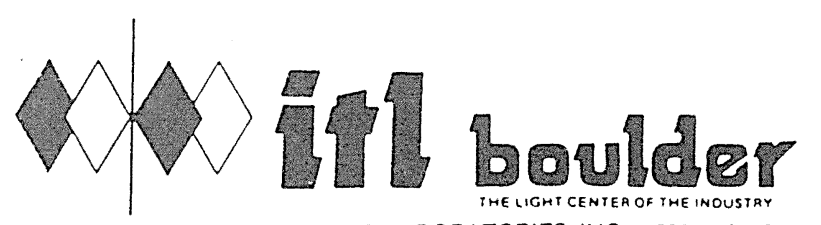

FAX (303) 449-52i: INDEPENDENT TESTING LABORATORIES, INC. • 3386 LONGHORN ROAD, BOULDER, COLORADO 80302 • PHONE (303) 442-1253

REPORT NUMBER: ITL40775

DATE : $7-26-1993$

PREPARED FOR: U.S. ARMY CORPS OF ENGINEERS

COEFFICIENTS OF UTILIZATION - ZONAI CAVITY METHOD

EFFECTIVE FLOOR CAVITY REFLECTANCE 0.20

\begin{tabular}{|c|c|c|c|c|c|c|c|c|c|c|c|c|c|c|c|c|c|c|}
\hline $\mathrm{RC}$ & \multicolumn{4}{|c|}{80} & \multicolumn{4}{|c|}{70} & \multicolumn{3}{|c|}{50} & \multicolumn{3}{|c|}{30} & \multicolumn{3}{|c|}{10} & 0 \\
\hline RW & 70 & 50 & 30 & 10 & 70 & 50 & 30 & 10 & 50 & 30 & 10 & 50 & 30 & 10 & 50 & 30 & 10 & 0 \\
\hline 0 & 52 & 52 & 52 & 52 & 51 & 51 & 51 & 51 & 49 & 49 & 49 & 47 & 47 & 47 & 45 & 45 & 45 & 4 \\
\hline 1 & 49 & 47 & 45 & 44 & 47 & 46 & 45 & 43 & 44 & 43 & 42 & 42 & 42 & 41 & 41 & 40 & 39 & 39 \\
\hline 2 & 45 & 42 & 39 & 37 & 44 & 41 & 39 & 37 & 39 & 37 & 36 & 38 & 36 & 35 & 37 & 35 & 34 & 33 \\
\hline 3 & 41 & 37 & 34 & 31 & 40 & 36 & 33 & 31 & 35 & 33 & 31 & 34 & 32 & 30 & 33 & 31 & 30 & 29 \\
\hline 4 & 38 & 33 & 29 & 27 & 37 & 32 & 29 & 27 & 31 & 29 & 26 & 30 & 28 & 26 & 29 & 27 & 26 & 25 \\
\hline 5 & 35 & 29 & 26 & 23 & 34 & 29 & 26 & 23 & 28 & 25 & 23 & 27 & 25 & 23 & 27 & 24 & 22 & 21 \\
\hline 6 & 32 & 27 & 23 & 20 & 31 & 26 & 23 & 20 & 25 & 22 & 20 & 25 & 22 & 20 & 24 & 21 & 20 & 19 \\
\hline 7 & 30 & 24 & 20 & 18 & 29 & 24 & 20 & 18 & 23 & 20 & 18 & 22 & 20 & 17 & 22 & 19 & 17 & 6 \\
\hline 8 & 27 & 22 & 18 & 16 & 27 & 21 & 18 & 16 & 21 & 18 & 16 & 20 & 18 & 15 & 20 & 17 & 15 & \\
\hline 0 & 26 & 20 & 16 & 14 & 25 & 20 & 16 & 14 & 19 & 16 & 14 & 19 & 16 & 14 & 18 & 16 & 14 & \\
\hline 10 & 24 & 18 & 15 & 13 & 23 & 18 & 15 & 13 & 18 & 15 & 12 & 17 & 14 & 12 & 17 & 14 & 12 & 12 \\
\hline
\end{tabular}

ALL CANDELA, LUMENS, LUMINANCE, COEFFICIENT OF UTIIIZATION AND VCP VALUES IN THIS REPORT ARE BASED ON RELATIVE PHOTOMETRY WHICH ASSUMES A BALLAST FACTOR OF 1.000. ANY CALCULATIONS PREPARED FROM THESE DATA SHOULD INCLUDE AN APPROPRIATE BALLAST FACTOR. 


\section{itl boulder}

FAX (303) 449-527:

INDEPENDENT TESTING LABORATORIES, INC. • 3386 LONGHORN ROAD, BOULDER, COLORADO 80302 • PHONE.(303) 442-1255 REPORT NUMBER: ITL40776

PREPARED FOR: U.S. ARMY CORPS OF ENGINEERS

CATALOG NUMBER: SHALLOW CAN, HORIZONTAL LAMP

LUMINAIRE: FABRICATED METAI FRAME，SPUN SPECULAR ALUMINUM REFLECTOR, WHITE PLASTIC TRIM RING, OPEN BOTTOM.

LAMP: 13 -WATT DOUBLE TWIN TUBE COMPACT FLUORESCENT, RATED 860 LUMENS, HORIZONTAL POSITION.

BALLAST: ROBERTSON HP-SI

MOUNTING : RECESSED

TOTAL INPUT WATTS $=16.0$ AT 120.0 VOLTS

THE O DEGREE PLANE IS OPPOSITE THE SOCKET.

LAMP CALIBRATION POSITION: HORIZONTAL $(* *$ explanation to follow **)

DATE: 7-28-1993

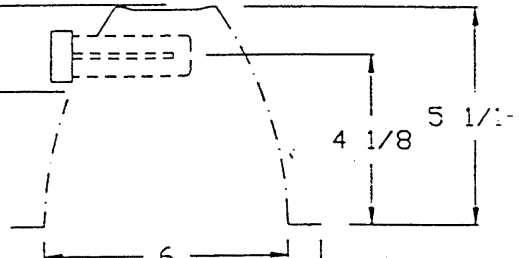

CANDELA DISTRIBUTION

$\begin{array}{rrrrrr} & 0.0 & 45.0 & 90.0 & 135.0 & 180.0 \\ 0 & 320 & 320 & 320 & 320 & 320 \\ 5 & 335 & 333 & 324 & 317 & 313 \\ 15 & 384 & 359 & 297 & 270 & 238 \\ 25 & 409 & 353 & 306 & 197 & 153 \\ 35 & 380 & 348 & 326 & 176 & 150 \\ 45 & 132 & 192 & 191 & 131 & 109 \\ 55 & 5 & 8 & 14 & 16 & 16 \\ 65 & 0 & 1 & 1 & 1 & 1 \\ 75 & 0 & 0 & 0 & 0 & 0 \\ 85 & 0 & 0 & 0 & 0 & 0 \\ 90 & 0 & 0 & 0 & 0 & 0\end{array}$

ZONAL LUMEN SUMMARY

$\begin{array}{rr}\text { ZONE } & \text { LUMENS } \\ 0-30 & 251 \\ 0-40 & 426 \\ 0-60 & 563 \\ 0-90 & 564 \\ 90-180 & 0 \\ 0-180 & 564\end{array}$

$$
\begin{array}{r}
\text { 8LAMP } \\
29.2 \\
49.5 \\
65.5 \\
65.6 \\
0.0 \\
65.6
\end{array}
$$

FLUX

\&FIXT

44.6

75.4

99.8

100.0

0.0

100.0

31
88
133
174
121
16
1
0
0

$65.6 \%$

TOTAI LUMINAIRE EFFICIENCY = CIE TYPE - DIRECT

PLANE

SPACING CRITERIA :

$$
\text { : } 0-D E G
$$

90-DEG 180-DEG

LUMINOUS DIAMETER:

$\begin{array}{ll}1.6 & 1.5 \\ 6.000 & \end{array}$

0.7

\begin{tabular}{rrrr} 
LUMINANCE DATA & \multicolumn{2}{c}{ IN -CANDELA/SQ M } \\
ANGLE & AVERAGE & AVERAGE & AVERAGE \\
IN DEG & $0-D E G$ & $90-D E G$ & $180-D E G$ \\
45 & 10230. & 14802. & 8447. \\
55 & 478. & 1338. & 1529. \\
65 & 0. & 130. & 130. \\
75 & 0. & 0. & 0. \\
85 & 0. & 0. & 0.
\end{tabular}

ANGIE

0-DEG $90-\mathrm{DEG}$ 180-DEG

$\begin{array}{lll}36076 . & 39505 . & 34160 . \\ 11045 . & 12738 . & 15900 .\end{array}$

$\begin{array}{rrrr}55 & 11045 . & 12738 . & 15900 . \\ 65 & 74 . & 155 . & 604 .\end{array}$

75

36 .

60 .

63.

17.22.

MAX/AVG MAX/AVG MAX/AVG

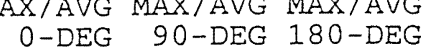

$\begin{array}{lll}3.5 & 2.7 & 4.0\end{array}$

23.1

0.0

0.0

9. 5

10.4

0.0

1. 2

4.6

0.0

0.0

THIS REPORT IS BASED ON PUBLISHED INDUSTRY PROCEDURES. FIELD PERFORMANCE MAY DIFFER FROM LABORATORY PERFORMANCE 


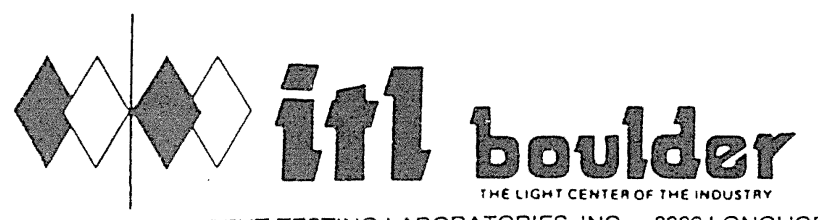

INDEPENDENT TESTING LABORATORIES, INC. • 3386 LONGHORN ROAD, BOULDER, COLORADO 80302 • PHONE (303) 442-125

FAX (303) 449-527:

REPORT NUMBER: ITL40776

PREPARED FOR: U.S. ARMY CORPS OF ENGINEERS

DATE: $7-28-1993$

\begin{tabular}{rrrrrrrrrrr} 
CANDELA. & \multicolumn{1}{l}{ DISTRIBUTION } \\
& 0.0 & 22.5 & 45.0 & 67.5 & 90.0 & 112.5 & 135.0 & 157.5 & 180.0 \\
0.0 & 320 & 320 & 320 & 320 & 320 & 320 & 320 & 320 & 320 \\
2.5 & 322 & 322 & 321 & 324 & 318 & 317 & 320 & 319 & 318 \\
5.0 & 335 & 335 & 333 & 335 & 324 & 318 & 317 & 315 & 313 \\
7.5 & 348 & 347 & 343 & 341 & 326 & 317 & 312 & 309 & 304 \\
10.0 & 360 & 358 & 348 & 336 & 316 & 304 & 301 & 299 & 291 \\
12.5 & 373 & 369 & 354 & 331 & 304 & 292 & 286 & 277 & 269 \\
15.0 & 384 & 379 & 359 & 327 & 297 & 283 & 270 & 244 & 238 \\
17.5 & 392 & 386 & 366 & 331 & 300 & 277 & 251 & 218 & 200 \\
20.0 & 398 & 387 & 363 & 334 & 298 & 267 & 234 & 195 & 164 \\
22.5 & 399 & 384 & 361 & 347 & 302 & 262 & 217 & 166 & 149 \\
25.0 & 409 & 376 & 353 & 348 & 306 & 256 & 197 & 156 & 153 \\
27.5 & 420 & 370 & 354 & 350 & 317 & 260 & 186 & 152 & 165 \\
30.0 & 423 & 368 & 370 & 354 & 326 & 263 & 177 & 152 & 167 \\
32.5 & 413 & 363 & 360 & 384 & 342 & 268 & 174 & 148 & 161 \\
35.0 & 380 & 359 & 348 & 362 & 326 & 265 & 176 & 140 & 150 \\
37.5 & 333 & 337 & 328 & 338 & 308 & 257 & 186 & 138 & 136 \\
40.0 & 269 & 291 & 306 & 299 & 264 & 236 & 189 & 141 & 123 \\
42.5 & 198 & 227 & 266 & 263 & 229 & 197 & 162 & 135 & 113 \\
45.0 & 132 & 158 & 192 & 201 & 191 & 161 & 131 & 118 & 109 \\
47.5 & 77 & 97 & 121 & 137 & 137 & 124 & 105 & 96 & 90 \\
50.0 & 42 & 55 & 66 & 76 & 81 & 75 & 69 & 62 & 61 \\
52.5 & 19 & 24 & 26 & 33 & 39 & 39 & 38 & 35 & 35 \\
55.0 & 5 & 6 & 8 & 12 & 14 & 15 & 16 & 16 & 16 \\
57.5 & 2 & 2 & 3 & 4 & 4 & 4 & 5 & 6 & 6 \\
60.0 & 1 & 2 & 2 & 2 & 3 & 3 & 3 & 3 & 3 \\
62.5 & 1 & 1 & 1 & 1 & 1 & 1 & 2 & 2 & 2 \\
65.0 & 0 & 1 & 1 & 1 & 1 & 1 & 1 & 1 & 1 \\
67.5 & 0 & 0 & 1 & 1 & 1 & 1 & 1 & 1 & 0 \\
70.0 & 0 & 0 & 1 & 1 & 1 & 1 & 1 & 1 & 0 \\
72.5 & 0 & 0 & 1 & 1 & 1 & 1 & 1 & 1 & 0 \\
75.0 & 0 & 0 & 0 & 0 & 0 & 0 & 0 & 0 & 0 \\
77.5 & 0 & 0 & 0 & 0 & 0 & 0 & 0 & 0 & 0 \\
80.0 & 0 & 0 & 0 & 0 & 0 & 0 & 0 & 0 & 0 \\
82.5 & 0 & 0 & 0 & 0 & 0 & 0 & 0 & 0 & 0 \\
85.0 & 0 & 0 & 0 & 0 & 0 & 0 & 0 & 0 & 0 \\
87.5 & 0 & 0 & 0 & 0 & 0 & 0 & 0 & 0 & 0 \\
90.0 & 0 & 0 & 0 & 0 & 0 & 0 & 0 & 0 & 0
\end{tabular}




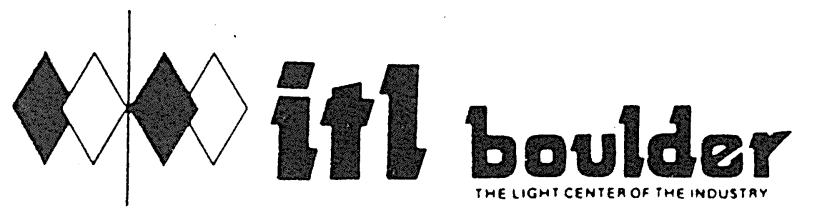

FAX (3C3) $449-52$ INDEPENDENT TESTING LABORATORIES, INC. • 3386 LONGHORN ROAD, BOULDER, COLORADO 80302 •PHONE (303) 442-1253

REPORT NUMBER: ITL40776

PREPARED FOR: U.S. ARMY CORPS OF ENGINEERS

DATE : $7-28-1993$

$\begin{array}{cr}\text { ZONAL LUMEN SUMMARY } \\ 0-5 & 8 . \\ 5-10 & 23 . \\ 10-15 & 38 . \\ 15-20 & 50 . \\ 20-25 & 61 . \\ 25-30 & 72 . \\ 30-35 & 86 . \\ 35-40 & 89 . \\ 40-45 & 76 . \\ 45-50 & 45 . \\ 50-55 & 14 . \\ 55-60 & 2 . \\ 60-65 & 1 . \\ 65-70 & 0 . \\ 70-75 & 0 . \\ 75-80 & 0 . \\ 80-85 & 0 . \\ 85-90 & 0 .\end{array}$




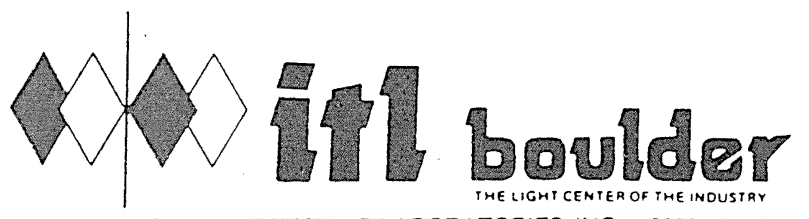

INDEPENDENT TESTING LABORATORIES. INC. • 3386 LONGHORN ROAD. BOULDER, COLORADO 80302 • PHONE (303) 442-i25

REPORT NUMBER: ITL40776

PREPARED FOR: U.S. ARMY CORPS OF ENGINEERS

DATE : $7-28-1993$

\section{** IMPORTANT ***}

The compact fluorescent lamps of the type used in this report require special attention in photometry and luminaire application. Specifically, the lamps generate lower flux output when operated in the horizontal position than when operated in the vertical position. Unfortunately, at the time of this report, only the vertical flux output (Iumens) is available from lamp manufacturers.

It is critical to note that, all else equal, a horizontal lamp calibration will yield higher luminaire candela and efficiency than a vertical lamp calibration. However, for a report which was generated using a horizontal lamp calibration, any application calculations should use the actual flux output (Iumens) from a horizontal lamp -- at this time, no such published lumen figures are available. For a report which was generated using a vertical lamp calibration, the flux output from a vertical lamp should be used. The published lamp lumen figure given on this report is for a vertical lamp. 


\section{itl boulder}

INDEPENDENT TESTING LABORATORIES, INC • 3386 LONGHORN ROAD. BOULDER COLORADO 80302 • PH

$\operatorname{FAX}(303) 4 \div 9-52$

REPORT NUMBER: ITL40776

DATE: $7-28-1993$

PREPARED FOR: U.S. ARMY CORPS OF ENGINEERS

COEFFICIENTS OF UTILIZATION - ZONAL CAVITY METHOD

EFFECTIVE FLOOR CAVITY REFLECTANCE 0.20

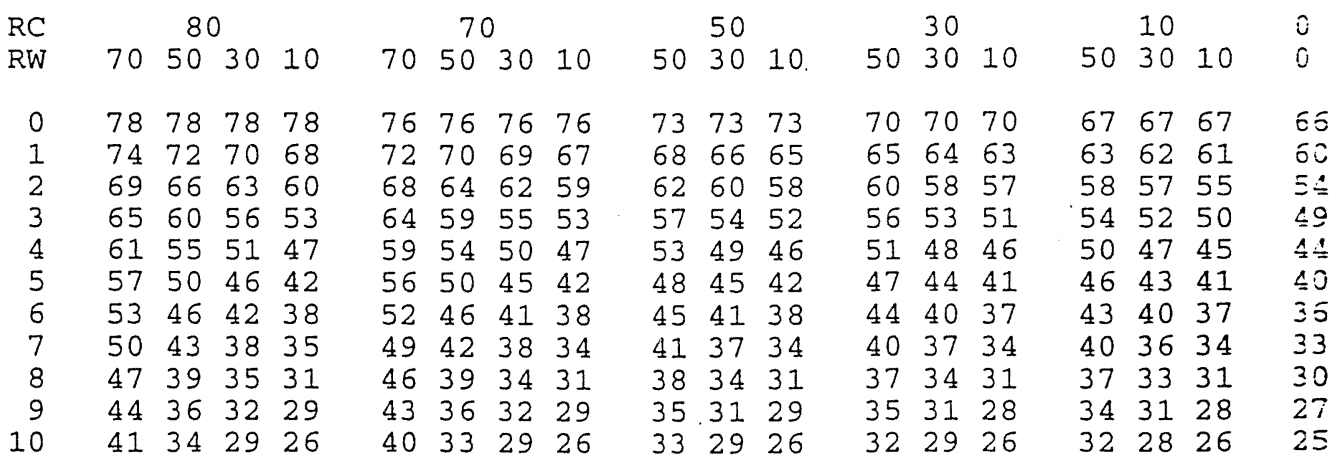

ALL CANDELA, LUMENS, LUMINANCE, COEFFICIENT OF UTILIZATION AND VCP VALUES IN THIS REPORT ARE BASED ON RELATIVE PHOTOMETRY WHICH ASSUMES A BALLAST FACTOR OF 1.000. ANY CALCULATIONS PREPARED FROM THESE DATA SHOULD INCLUDE AN APPROPRIATE BALLAST FACTOR. 


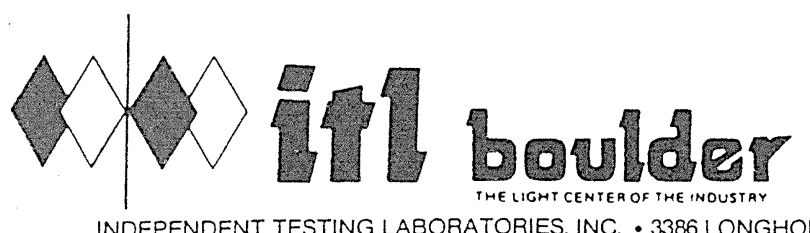

INDEPENDENT TESTING LABORATORIES, INC. • 3386 LONGHORN ROAD, BOULDER. COLORADO 80302 • PHONE (303) 442-125:

REPORT NUMBER: ITL40777

PREPARED FOR: U.S. ARMY CORPS OF ENGINEERS

DATE: $7-21-1993$

CATALOG NUMBER: SHALLOW CAN, SHORT LAMP

LUMINAIRE: FABRICATED SQUARE METAL HOUSING, SPUN SPECULAR ALUMINUM REFLECTOR, WHITE PAINTED METAL TRIM RING, OPEN BOTTOM.

LAMP: 15-WATT FLB15/TL COMPACT FLUORESCENT, RATED 700 LUMENS, VERTICFI. BASE-UP POSITION.

MOUNTING: RECESSED

TOTAL INPUT WATTS $=16.3$ AT 120.0 VOLTS

CANDELA DISTRIBUTION

$\begin{array}{rrrrrr} & 0.0 & 45.0 & 90.0 & 135.0 & 180.0 \\ 0 & 128 & 128 & 128 & 128 & 128 \\ 5 & 138 & 136 & 129 & 122 & 121 \\ 15 & 170 & 156 & 133 & 123 & 121 \\ 25 & 200 & 175 & 142 & 137 & 141 \\ 35 & 208 & 179 & 144 & 139 & 145 \\ 45 & 179 & 156 & 132 & 128 & 132 \\ 55 & 133 & 118 & 107 & 103 & 106 \\ 65 & 86 & 79 & 72 & 70 & 71 \\ 75 & 42 & 40 & 36 & 36 & 37 \\ 85 & 6 & 7 & 7 & 7 & 7 \\ 90 & 0 & 0 & 0 & 0 & 0\end{array}$

ZONAL LUMEN SUMMARY

$\begin{array}{rrr}\text { ZONE } & \text { LUMENS } & \text { 8LAMP } \\ 0-30 & 124 & 17.8 \\ 0-40 & 224 & 32.0 \\ 0-60 & 435 & 62.1 \\ 0-90 & 557 & 79.6 \\ 90-180 & 0 & 0.0 \\ 0-180 & 557 & 79.6\end{array}$

FLUX

12

40

141272

100

110

100

74

40

8

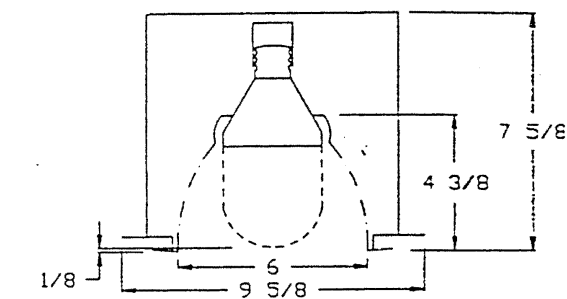

TOTAL LUMINAIRE EFFICIENCY = CIE TYPE - DIRECT

PLANE

: $0-D E G$ 90-DEG 180-DEG

SPACING CRITERIA : $2.0 \quad 1.7 \quad 1.7$

LUMINOUS DIAMETER:

$$
6.000
$$

FIXT
22.3
40.3
78.1
100.0
0.0
100.0

79.6 웅

LUMINANCE DATA IN CANDELA/SQ M ANGLE AVERAGE AVERAGE AVERAGE IN DEG O-DEG 90-DEG 180-DEG

45 13872. 10230. 10230 .

5512707.10223 .10127$.

65 11151. 9336. 9206.

75 8893. 7622.7834.

85 3773. 4401. 4401.

ANGLE MAXIMUM MAXIMUM MAXIMUM MAX/AVG MAX/AVG MAX/AVG

IN DEG 0-DEG 90-DEG 180-DEG 0-DEG 90-DEG $180-D E G$

45 18196. 17047. 19021. 1.3 .91 .7

55 17083. 16329. 17873. $1.31 .3 \quad 1.6 \quad 1.8$

65 15899. 15181. 15863. $1.41 .6 \quad 1.6$

75 14320. 12023. 12920. $1.6 \quad 1.6 \quad 1.6$

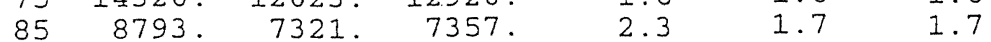

THIS REPORT IS BASED ON PUBLISHED INDUSTRY PROCEDURES. FIELD PERFORMANCE MAY DIFFER FROM LABORATORY PERFOEMANC: 


\section{itl boulder}

INDEPENDENT TESTING LABORATORIES, INC. • 3386 LONGHORN ROAD, BOULDER. COLORADO 80302 • PHONE (303) 442-12

REPORT NUMBER: ITL40777

PREPARED FOR: U.S. ARMY CORPS OF ENGINEERS

DATE: $7-21-1993$

CANDELA DISTRIBUTION

$\begin{array}{rrrrrrrrrrr}0.0 & 128 & 128 & 128 & 128 & 128 & 128 & 128 & 128 & 128 \\ 2.5 & 133 & 133 & 132 & 131 & 129 & 127 & 126 & 124 & 124 \\ 5.0 & 138 & 138 & 136 & 132 & 129 & 125 & 122 & 121 & 121 \\ 7.5 & 145 & 145 & 141 & 135 & 130 & 125 & 122 & 119 & 118 \\ 10.0 & 154 & 152 & 146 & 139 & 130 & 125 & 120 & 118 & 116 \\ 12.5 & 161 & 159 & 152 & 141 & 131 & 124 & 121 & 119 & 119 \\ 15.0 & 170 & 166 & 156 & 143 & 133 & 126 & 123 & 121 & 121 \\ 17.5 & 178 & 172 & 162 & 147 & 135 & 128 & 127 & 126 & 125 \\ 20.0 & 185 & 180 & 165 & 149 & 137 & 130 & 129 & 130 & 131 \\ 22.5 & 193 & 186 & 170 & 152 & 139 & 134 & 133 & 135 & 135 \\ 25.0 & 200 & 193 & 175 & 155 & 142 & 136 & 137 & 140 & 141 \\ 27.5 & 207 & 198 & 178 & 156 & 143 & 139 & 139 & 142 & 145 \\ 30.0 & 210 & 200 & 180 & 158 & 143 & 139 & 141 & 143 & 145 \\ 32.5 & 209 & 202 & 180 & 158 & 144 & 140 & 140 & 143 & 146 \\ 35.0 & 208 & 201 & 179 & 156 & 144 & 139 & 139 & 142 & 145 \\ 37.5 & 205 & 198 & 177 & 155 & 143 & 138 & 139 & 141 & 144 \\ 40.0 & 197 & 191 & 170 & 151 & 140 & 136 & 136 & 139 & 140 \\ 42.5 & 189 & 184 & 164 & 147 & 137 & 133 & 133 & 134 & 136 \\ 45.0 & 179 & 175 & 156 & 140 & 132 & 128 & 128 & 130 & 132 \\ 47.5 & 168 & 164 & 148 & 134 & 127 & 124 & 123 & 124 & 127 \\ 50.0 & 155 & 153 & 137 & 126 & 121 & 118 & 117 & 118 & 121 \\ 52.5 & 144 & 142 & 128 & 118 & 114 & 112 & 111 & 112 & 113 \\ 55.0 & 133 & 131 & 118 & 110 & 107 & 104 & 103 & 104 & 106 \\ 57.5 & 121 & 119 & 108 & 100 & 98 & 96 & 95 & 97 & 98 \\ 60.0 & 109 & 109 & 98 & 91 & 90 & 87 & 87 & 88 & 89 \\ 62.5 & 96 & 97 & 88 & 83 & 81 & 79 & 79 & 80 & 80 \\ 65.0 & 86 & 87 & 79 & 73 & 72 & 70 & 70 & 72 & 71 \\ 67.5 & 75 & 75 & 69 & 64 & 63 & 62 & 62 & 63 & 63 \\ 70.0 & 64 & 64 & 59 & 54 & 53 & 53 & 53 & 54 & 54 \\ 72.5 & 52 & 53 & 49 & 46 & 44 & 44 & 45 & 45 & 46 \\ 75.0 & 42 & 42 & 40 & 37 & 36 & 36 & 36 & 36 & 37 \\ 77.5 & 31 & 32 & 31 & 28 & 27 & 28 & 28 & 28 & 28 \\ 80.0 & 22 & 23 & 22 & 20 & 20 & 20 & 20 & 20 & 21 \\ 82.5 & 14 & 15 . & 14 & 13 & 13 & 13 & 13 & 13 & 13 \\ 85.0 & 6 & 7 & 7 & 7 & 7 & 7 & 7 & 6 & 7 \\ 87.5 & 1 & 1 & 1 & 1 & 1 & 1 & 1 & 1 & 1 \\ 90.0 & 0 & 0 & 0 & 0 & 0 & 0 & 0 & 0 & 0\end{array}$




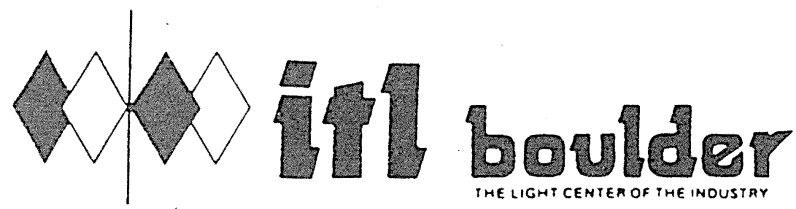

FAX (303) 449-527: INDEPENDENT TESTING LABORATORIES. INC. • 3386 LONGHORN ROAD, BOULDER, COLORADO 80302 •PHONE (303) 442-125:

REPORT NUMBER: ITL40777

PREPARED FOR: U.S. ARMY CORPS OF ENGINEERS

DATE: $7-21-1993$

$\begin{array}{cr}\text { ZONAL LUMEN } & \text { SUMMARY } \\ 0-5 & 3 . \\ 5-10 & 9 . \\ 10-15 & 16 . \\ 15-20 & 24 . \\ 20-25 & 32 . \\ 25-30 & 40 . \\ 30-35 & 47 . \\ 35-40 & 53 . \\ 40-45 & 55 . \\ 45-50 & 55 . \\ 50-55 & 52 . \\ 55-60 & 48 . \\ 60-65 & 41 . \\ 65-70 & 33 . \\ 70-75 & 25 . \\ 75-80 & 15 . \\ 80-85 & 7 . \\ 85-90 & 1 .\end{array}$




\title{
itl boulder
}

INDEPENDENT TESTING LABORATORIES, INC. • 3386 LONGHORN ROAD, BOULDER, COLORADO 80302 • PHONE (303) 442-1253

REPORT NUMBER: ITL40777

DATE : 7-21-1993

PREPARED FOR: U.S. ARMY CORPS OF ENGINEERS

\author{
COEFFICIENTS OF UTIIIZATION - ZONAI CAVITY'METHOD \\ EFFECTIVE FLOOR CAVITY REFLECTANCE 0.20
}

\begin{tabular}{|c|c|c|c|c|c|c|c|c|c|c|c|c|c|c|c|c|c|}
\hline $\mathrm{RC}$ & \multicolumn{3}{|c|}{80} & \multicolumn{4}{|c|}{70} & \multicolumn{3}{|c|}{50} & \multicolumn{3}{|c|}{30} & \multicolumn{3}{|c|}{10} & 0 \\
\hline RW & $70 \quad 50$ & 30 & 10 & 70 & 50 & 30 & 10 & 50 & 30 & 10 & 50 & 30 & 10 & 50 & 30 & 10 & 0 \\
\hline 0 & 9595 & 95 & 95 & 92 & 92 & 92 & 92 & 88 & 88 & 88 & 84 & 84 & 84 & 81 & 81 & 81 & 79 \\
\hline 1 & $86 \quad 82$ & 79 & 76 & 84 & 80 & 77 & 74 & 77 & 74 & 72 & 74 & 72 & 70 & 71 & 69 & 68 & 66 \\
\hline 2 & $\begin{array}{ll}78 & 71\end{array}$ & 66 & 61 & 76 & 70 & 65 & 60 & 67 & 63 & 59 & 64 & 61 & 57 & 62 & 59 & 56 & \\
\hline 3 & $\begin{array}{ll}71 & 62\end{array}$ & 55 & 50 & 69 & 61 & 55 & 49 & 58 & 53 & 49 & 56 & 52 & 48 & 54 & 50 & 47 & 5 \\
\hline 4 & $64 \quad 54$ & 47 & 42 & 63 & 53 & 47 & 41 & 51 & 45 & 41 & 50 & 44 & 40 & 48 & 43 & 40 & 8 \\
\hline 5 & 59 & 41 & 35 & 57 & 47 & 40 & 35 & 46 & 40 & 35 & 44 & 39 & 34 & 43 & 38 & 34 & 2 \\
\hline 6 & 54 & 36 & 30 & 53 & 42 & 35 & 30 & 41 & 35 & 30 & 40 & 34 & 30 & 38 & 33 & 29 & 8 \\
\hline 7 & 50 & 32 & 27 & 49 & 38 & 31 & 26 & 37 & 31 & 26 & 36 & 30 & 26 & 35 & 30 & 26 & \\
\hline 8 & $46 \quad 35$ & 28 & 23 & 45 & 35 & 28 & 23 & 34 & 27 & 23 & 33 & 27 & 23 & 32 & 27 & 23 & 1 \\
\hline 9 & $43 \quad 32$ & 25 & 21 & 42 & 32 & 25 & 21 & 31 & 25 & 21 & 30 & 24 & 20 & 29 & 24 & 20 & $y$ \\
\hline 10 & $40 \quad 29$ & 23 & 19 & 39 & 29 & 23 & 19 & 28 & 22 & 18 & 27 & 22 & 18 & 27 & 22 & 18 & 17 \\
\hline
\end{tabular}

ALL CANDELA, LUMENS, LUMINANCE, COEFFICIENT OF UTILIZATION AND VCP VALUES IN THIS REPORT ARE BASED ON RELATIVE PHOTOMETRY WHICH ASSUMES A BALLAST FACTOR OF 1.000. ANY CALCULATIONS PREPARED FROM THESE DATA SHOULD INCLUDE AN APPROPRIATE BALLAST FACTOR. 


\section{1til boulder}

$\operatorname{FAX}(303) 449-527:$ INDEPENDENT TESTING LABORATORIES, INC. • 3386 LONGHORN ROAD, BOULDER, COLORADO 80302 • PHONE (303) 442-125:

REPORT NUMBER: ITL40778

PREPARED FOR: U.S. ARMY CORPS OF ENGINEERS

DATE: $7-29-1993$

CATALOG NUMBER: SHALLOW CAN, SHORT REFLECTOR

LUMINAIRE: FABRICATED SQUARE METAL HOUSING, SPUN SPECULAR ALUMINUM

REFLECTOR, WHITE PAINTED METAL TRIM RING, OPEN BOTTOM.

LAMP: STANDARD GE A60 60-WATT A-19 INCANDESCENT, RATED 870 LUMENS, VERTICAL BASE-UP POSITION.

MOUNTING : RECESSED

$\begin{array}{rcr}\text { DEG } & \text { CANDELA } & \text { LUMENS } \\ 0 & 1015 & \\ 5 & 672 & 61 \\ 15 & 452 & 126 \\ 25 & 305 & 141 \\ 35 & 199 & 122 \\ 45 & 54 & 44 \\ 55 & 4 & 5 \\ 65 & 1 & 1 \\ 75 & 0 & 0 \\ 85 & 0 & 0 \\ 90 & 0 & \end{array}$

ZONAL LUMEN SUMMARY ZONE

$0-30$

LUMENS

$0-40$

328

$0-60$

$0-90$

$90-180$

$0-180$

501

$$
\begin{array}{r}
\text { 8LAMP } \\
37.7 \\
51.7 \\
57.4 \\
57.5 \\
0.0 \\
57.5
\end{array}
$$

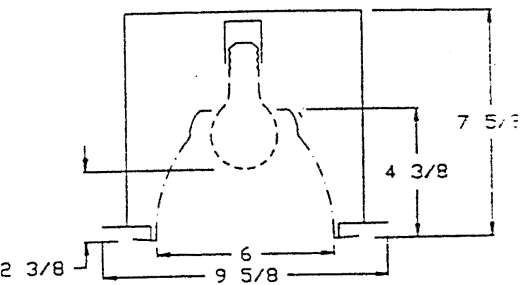

TOTAL LUMINAIRE EFFICIENCY = CIE TYPE - DIRECT

LUMINAIRE SPACING CRITERION $=0.4$

LUMINOUS DIAMETER: 6.000

LUMINANCE DATA IN CANDELA/SQ M ANGLE AVERAGE MAXIMUM MAX/AVG IN DEG

$\begin{array}{rrrr}45 & 4185 . & 26538 . & 6.3 \\ 55 & 382 . & 6385 . & 16.7 \\ 65 & 130 . & -130 . & 1.0 \\ 75 & 0 . & 32 . & 0.0 \\ 85 & 0 . & 18 . & 0.0\end{array}$
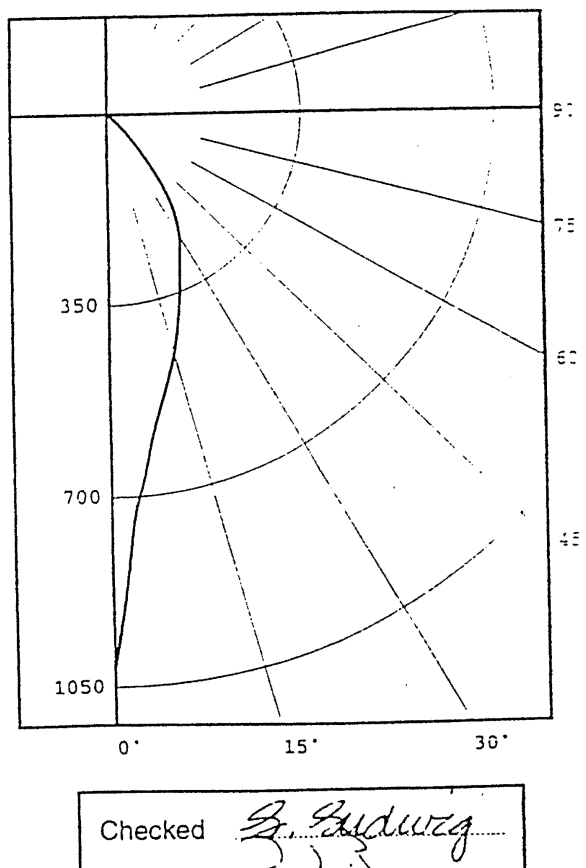

Approved (s) 


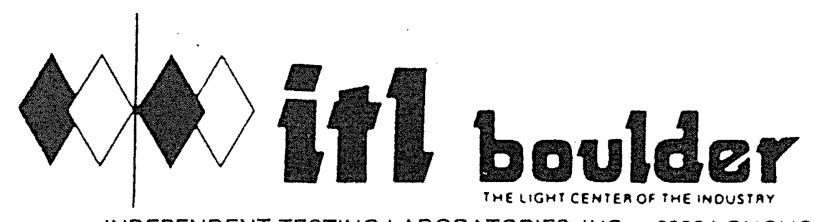

FAX (303) 449-5274 INDEPENDENT TESTING LABORATORIES, INC. • 3386 LONGHORN ROAD, BOULDER, COLORADO 80302 • PHONE (303) 442-1255

REPORT NUMBER: ITL 40778

PREPARED FOR: U.S. ARMY CORPS OF ENGINEERS

DATE: $7-29-1993$

$\begin{array}{rr}\text { CANDELA } & \text { DISTR } \\ & 0.0 \\ 0.0 & 1015 \\ 2.5 & 789 \\ 5.0 & 672 \\ 7.5 & 590 \\ 10.0 & 536 \\ 12.5 & 492 \\ 15.0 & 452 \\ 17.5 & 410 \\ 20.0 & 371 \\ 22.5 & 336 \\ 25.0 & 305 \\ 27.5 & 280 \\ 30.0 & 257 \\ 32.5 & 230 \\ 35.0 & 199 \\ 37.5 & 162 \\ 40.0 & 120 \\ 42.5 & 82 \\ 45.0 & 54 \\ 47.5 & 34 \\ 50.0 & 20 \\ 52.5 & 10 \\ 55.0 & 4 \\ 57.5 & 2 \\ 60.0 & 1 \\ 62.5 & 1 \\ 65.0 & 1 \\ 67.5 & 1 \\ 70.0 & 0 \\ 72.5 & 0 \\ 75.0 & 0 \\ 77.5 & 0 \\ 80.0 & 0 \\ 82.5 & 0 \\ 85.0 & 0 \\ 87.5 & 0 \\ 90.0 & 0 \\ & \\ 37 \\ 37\end{array}$

ZONAL LUMEN SUMMARY

$0-5$ 19.

$5-10 \quad 42$

$10-15 \quad 58$

$15-20 \quad 68$.

$20-25 \quad 70$.

$25-30 \quad 71$.

$30-35 \quad 68$.

$35-40 \quad 54$.

$40-4530$

$45-50 \quad 14$.

$50-554$

$55-60 \quad 1$

$60-65-0$

$65-70 \quad 1$

$70-75 \quad 0$

$75-80 \quad 0$.

$80-85 \quad 0$

$85-90 \quad 0$. 


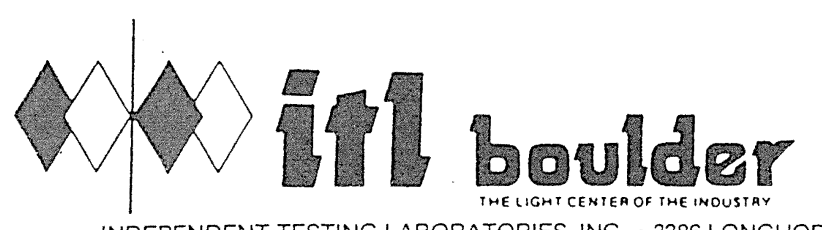

INDEPENDENT TESTING LABORATORIES, INC. • 3386 LONGHORN ROAD, BOULDER, COLORADO 80302 • PHONE (303) $442-12$

REPORT NUMBER: ITL4 0778

DATE: $7-29-1993$

PREPARED FOR: U.S. ARMY CORPS OF ENGINEERS

COEFFICIENTS OF UTILIZATION - ZONAL CAVITY METHOD

EFFECTIVE FLOOR CAVITY REFLECTANCE 0.20

\begin{tabular}{|c|c|c|c|c|c|c|c|c|c|c|c|c|c|c|c|c|c|c|}
\hline $\mathrm{RC}$ & \multicolumn{4}{|c|}{80} & \multicolumn{4}{|c|}{70} & \multicolumn{3}{|c|}{50} & \multicolumn{3}{|c|}{30} & \multicolumn{3}{|c|}{10} & 0 \\
\hline RW & 70 & 50 & 30 & 10 & 70 & 50 & 30 & 10 & 50 & 30 & 10 & 50 & 30 & 10 & 50 & 30 & 10 & 0 \\
\hline 0 & 68 & 68 & 68 & 68 & 67 & 67 & 67 & 67 & 64 & 64 & 64 & 61 & 61 & 61 & 59 & 59 & 59 & 7 \\
\hline 1 & 65 & 64 & 62 & 61 & 64 & 62 & 61 & 60 & 60 & 59 & 58 & 58 & ו & 56 & 56 & 55 & 55 & \pm \\
\hline 2 & 62 & 59 & 57 & 55 & 61 & 58 & 56 & 54 & 56 & 55 & 53 & 55 & 53 & 52 & 53 & 52 & 51 & 0 \\
\hline 3 & 59 & 55 & 52 & 50 & 58 & 54 & 52 & 49 & 53 & 50 & 49 & 51 & 49 & 48 & 50 & 48 & 47 & 6 \\
\hline 4 & 56 & 51 & 48 & 46 & 55 & 51 & 48 & 45 & 49 & 47 & 45 & 48 & 46 & 44 & 47 & 45 & 44 & 3 \\
\hline 5 & 53 & 48 & 45 & 42 & 52 & 47 & 44 & 42 & 46 & 44 & 41 & 45. & 43 & 41 & 44 & 42 & 41 & 0 \\
\hline 6 & 50 & 45 & 41 & 39 & 49 & 44 & 41 & 39 & 44 & 41 & 39 & 43 & 40 & 38 & 42 & 40 & 38 & 7 \\
\hline 7 & 48 & 42 & 39 & 36 & 47 & 42 & 38 & 36 & 41 & 38 & 36 & 40 & 38 & 36 & 40 & 37 & 36 & \\
\hline 8 & 45 & 40 & 36 & 34 & 44 & 39 & 36 & 34 & 39 & 36 & 34 & 38 & 35 & 33 & 38 & 35 & 33 & \\
\hline 9 & 43 & 37 & 34 & 32 & 42 & 37 & 34 & 32 & 37 & 34 & 31 & 36 & 33 & 31 & 36 & 33 & 31 & \\
\hline 10 & 41 & 35 & 32 & 30 & 40 & 35 & 32 & 30 & 35 & 32 & 30 & 34 & 1 & 30 & 34 & 31 & 29 & 9 \\
\hline
\end{tabular}

ALI CANDELA, LUMENS, LUMINANCE, COEFFICIENT OF UTILIZATION AND VCP VALUES IN THIS REPORT ARE BASED ON RELATIVE PHOTOMETRY WHICH ASSUMES A BALLAST FACTOR OF 1.000. ANY CALCULATIONS PREPARED FROM THESE DATA SHOULD INCLUDE AN APPROPRIATE BALLAST FACTOR. 


\section{ifl boulder}

INDEPENDENT TESTING LABORATORIES, INC. • 3386 LONGHORN ROAD, BOULDER, COLORADO 80302 • PHONE (303) 442-12 25

REPORT NUMBER: ITL40779

PREPARED FOR: U.S. ARMY CORPS OF ENGINEERS

CATALOG NUMBER: SHALLOW CAN, FULL REFLECTOR

LUMINAIRE: FABRICATED SQUARE METAL HOUSING, SPUN SPECULAR ALUMINUM REFLECTOR, WHITE PAINTED METAL TRIM RING, OPEN BOTTOM.

LAMP: 15-WATT FLB15/TL COMPACT FLUORESCENT, RATED 700 LUMENS, VERTICAI BASE-UP POSITION.

MOUNTING: RECESSED

TOTAL INPUT WATTS $=16.0$ AT 120.0 VOLTS

CANDELA DISTRIBUTION

$\begin{array}{rrrrrr}0 & 0.0 & 45.0 & 90.0 & 135.0 & 180.0 \\ 5 & 153 & 153 & 153 & 153 & 153 \\ 15 & 166 & 163 & 155 & 148 & 145 \\ 25 & 196 & 182 & 158 & 148 & 147 \\ 35 & 210 & 188 & 158 & 152 & 155 \\ 45 & 164 & 180 & 153 & 148 & 151 \\ 55 & 119 & 147 & 130 & 126 & 129 \\ 65 & 72 & 67 & 67 & 96 & 97 \\ 75 & 26 & 26 & 24 & 60 & 59 \\ 85 & 2 & 2 & 2 & 2 & 22 \\ 90 & 0 & 0 & 0 & 0 & 0\end{array}$

ZONAI LUMEN SUMMARY

$\begin{array}{rrr}\text { ZONE } & \text { LUMENS } & \text { 8LAMP } \\ 0-30 & 140 & 20.0 \\ 0-40 & 243 & 34.7 \\ 0-60 & 441 & 63.0 \\ 0-90 & 532 & 76.1 \\ 90-180 & 0 & 0.0 \\ 0-180 & 532 & 76.1\end{array}$

FLUX

TOTAL LUMINAIRE EFFICIENCY = CIE TYPE - DIRECT

$\begin{array}{llcrr}\text { PLANE } & \text { 0-DEG } & 90-\mathrm{DEG} & 180-\mathrm{DEG} \\ \text { SPACING CRITERIA : } & 1.8 & 1.5 & 1.5 \\ \text { LUMINOUS DIAMETER: } & 5.875 & & \end{array}$

LUMINANCE DATA IN CANDELA/SQ M ANGLE AVERAGE AVERAGE AVERAGE IN DEG 0-DEG 90-DEG 180-DEG 45 13256. 10507. 10427.

55 11858. 9665. 9665.

$659737 . \quad 8249.7979$.

75 5741. 5300. 4858 .

$851312 . \quad 1312.1312$.

DATE : $7-22-1993$

FAX (303) 449-52:-

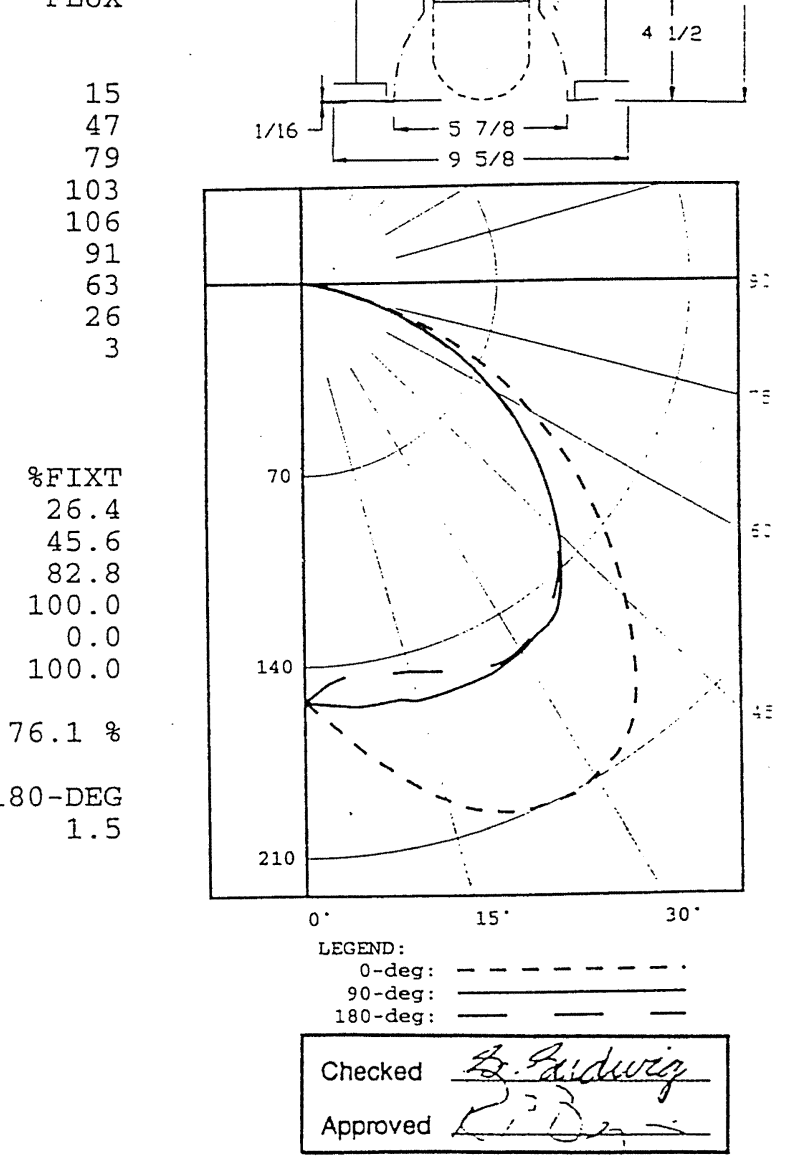

ANGLE MAXIMUM MAXIMUM MAXIMUM MAX/AVG MAX/AVG MAX/AVG

IN DEG 0-DEG 90-DEG 180-DEG 0-DEG 90-DEG 180-DEG

$\begin{array}{llllll}45 & 19595 & 17441 & 17514 & 1.5 & 1.7\end{array}$

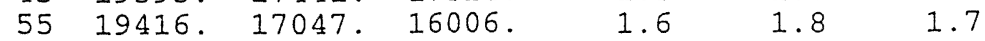

65 17334. 15863. 16078. $1.8 \quad 1.9 \quad 1.9 \quad 2.0$

75 13351. 12489. 12920. $2.3 \quad 2.4 \quad 2.7$

85 3790. 2017. 3094. 2.9 $2.5 \quad 1.5$

THIS REPORT IS BASED ON PUBLISHED INDUSTRY PROCEDURES. FIELD PERFORMANCE MAY DIFFER FROM LABORATORY PERFORMANC 


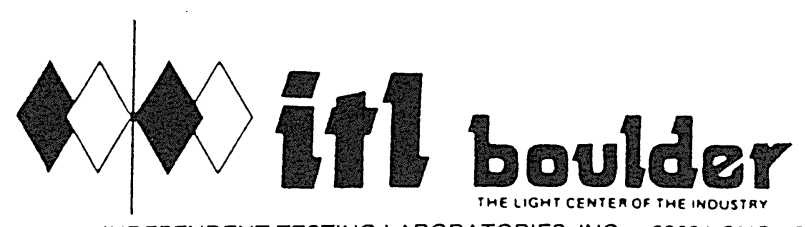

INDEPENDENT TESTING LABORATORIES, INC. • 3386 LONGHORN ROAD, BOULDER, COLORADO $80302 \cdot$ PH

REPORT NUMBER: ITL40779

PREPARED FOR: U.S. ARMY CORPS OF ENGINEERS

DATE : $7-22-1993$

CANDELA DISTRIBUTION

\begin{tabular}{|c|c|c|c|c|c|c|c|c|}
\hline 0.0 & 225 & 450 & & & 7125 & 1350 & 5 & 180 \\
\hline 153 & 153 & 153 & 153 & 153 & 153 & 153 & 153 & 153 \\
\hline 159 & 159 & 158 & 156 & 154 & 152 & 150 & 149 & 148 \\
\hline 166 & 165 & 163 & 159 & 155 & 151 & 148 & 146 & 145 \\
\hline 174 & 172 & 168 & 162 & 156 & 151 & 147 & 146 & 144 \\
\hline 181 & 179 & 173 & 165 & 156 & 150 & 147 & 145 & 145 \\
\hline 189 & 186 & 178 & 167 & 156 & 150 & 147 & 147 & 146 \\
\hline 196 & 192 & 182 & 169 & 158 & 151 & 148 & 148 & 147 \\
\hline 202 & 197 & 185 & 170 & 158 & 151 & 149 & 150 & 149 \\
\hline 206 & 201 & 186 & 170 & 158 & 151 & 150 & 151 & 151 \\
\hline 209 & 203 & 188 & 170 & 158 & 151 & 151 & 153 & 153 \\
\hline 210 & 205 & 188 & 170 & 158 & 152 & 152 & 155 & 155 \\
\hline 211 & 205 & 188 & 169 & 157 & 152 & 152 & 155 & 156 \\
\hline 209 & 204 & 186 & 168 & 156 & 151 & 152 & 154 & 155 \\
\hline 207 & 201 & 183 & 166 & 154 & 150 & 150 & 152 & 153 \\
\hline 203 & 198 & 180 & 163 & 153 & 148 & 148 & 149 & 151 \\
\hline 196 & 192 & 175 & 159 & 150 & 145 & 144 & 146 & 47 \\
\hline 186 & 183 & 167 & 152 & 144 & 140 & 139 & 141 & 42 \\
\hline 175 & 173 & 157 & 144 & 137 & 134 & 133 & 135 & 136 \\
\hline 164 & 162 & 147 & 135 & 130 & 127 & 126 & 128 & 129 \\
\hline 153 & 151 & 137 & 126 & 122 & 119 & 119 & 121 & 122 \\
\hline 142 & 140 & 127 & 117 & 114 & 112 & 111 & 114 & 114 \\
\hline 130 & 129 & 117 & 108 & 106 & 104 & 104 & 106 & 106 \\
\hline 119 & 118 & 107 & 99 & 97 & 96 & 96 & 98 & 97 \\
\hline 107 & 106 & 97 & 90 & 89 & 87 & 87 & 89 & 88 \\
\hline 95 & 95 & 87 & 81 & 79 & 79 & 79 & 79 & 79 \\
\hline 84 & 83 & 77 & 72 & 70 & 70 & 70 & 70 & 69 \\
\hline 72 & 71 & 67 & 62 & 61 & 61 & 60 & 60 & 59 \\
\hline 60 & 60 & 56 & 53 & 51 & 51 & 51 & 50 & 50 \\
\hline 49 & 48 & 45 & 43 & 42 & 42 & 41 & 40 & 40 \\
\hline 37 & 37 & 35 & 34 & 33 & 33 & 32 & 30 & 30 \\
\hline 26 & 27 & 26 & 25 & 24 & 24 & 23 & 22 & 22 \\
\hline 17 & 18 & 17 & 17 & 17 & 16 & 15 & 14 & 14 \\
\hline 10 & 10 & 9 & 10 & 10 & 9 & 9 & 8 & 8 \\
\hline 5 & 5 & 5 & 5 & 5 & 5 & 5 & 4 & 4 \\
\hline 2 & 2 & 2 & 2 & 2 & 2 & 2 & 2 & 2 \\
\hline 5 & 0 & 0 & 0 & 0 & 1 & 1 & 1 & 1 \\
\hline 0 & 0 & 0 & 0 & 0 & 0 & 0 & 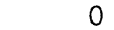 & \\
\hline
\end{tabular}

THIS REPORT IS BASED ON PUBLISHED INDUSTRY PROCEDURES. FIELD PERFORMANCE MAY DIFFER FRCM LABORATORY PERFORMANC: 


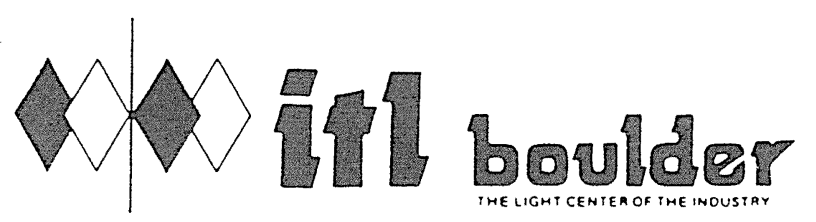

FAX (303) 449-52: INDEPENDENT TESTING LABORATORIES, INC. • 3386 LONGHORN ROAD, BOULDER. COLORADO 80302 • PHONE (303) 442-125.

REPORT NUMBER: ITL 40779

PREPARED FOR: U.S. ARMY CORPS OF ENGINEERS

DATE : $7-22-1993$

$\begin{array}{cr}\text { ZONAL LUMEN SUMMARY } \\ 0-5 & 4 . \\ 5-10 & 11 . \\ 10-15 & 19 . \\ 15-20 & 28 . \\ 20-25 & 36 . \\ 25-30 & 43 . \\ 30-35 & 49 . \\ 35-40 & 53 . \\ 40-45 & 54 . \\ 45-50 & 52 . \\ 50-55 & 48 . \\ 55-60 & 43 . \\ 60-65 & 36 . \\ 65-70 & 27 . \\ 70-75 & 17 . \\ 75-80 & 9 . \\ 80-85 & 3 . \\ 85-90 & 0 .\end{array}$




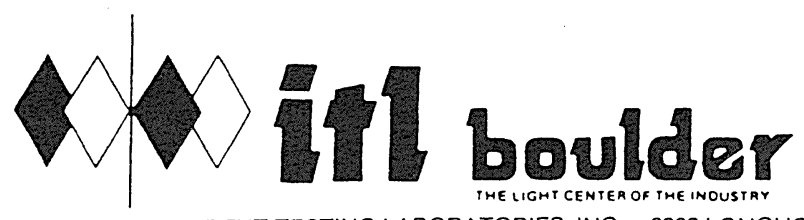

INDEPENDENT TESTING LABORATORIES, INC. • 3386 LONGHORN ROAD. BOULDER, COLORADO 80302 • PHONE (303) 442-125ः

REPORT NUMBER: ITL40779

DATE: $7-22-1993$

PREPARED FOR: U.S. ARMY CORPS OF ENGINEERS

COEFFICIENTS OF UTILIZATION - ZONAL CAVITY METHOD

EFFECTIVE FLOOR CAVITY REFLECTANCE 0.20

\begin{tabular}{|c|c|c|c|c|c|c|c|c|c|c|c|c|c|c|c|c|c|c|}
\hline $\mathrm{RC}$ & \multicolumn{4}{|c|}{80} & \multicolumn{4}{|c|}{70} & \multicolumn{3}{|c|}{50} & \multicolumn{3}{|c|}{30} & \multicolumn{3}{|c|}{10} & 0 \\
\hline $\mathrm{RW}$ & 70 & 50 & 30 & 10 & 70 & 50 & 30 & 10 & 50 & 30 & 10 & 50 & 30 & 10 & 50 & 30 & 10 & 0 \\
\hline 0 & 91 & 91 & 91 & 91 & 89 & 89 & 89 & 89 & 85 & 85 & 85 & 81 & 81 & 81 & 78 & 78 & 78 & 6 \\
\hline 1 & 83 & 80 & 77 & 74 & 81 & 78 & 76 & 73 & 75 & 73 & 71 & 72 & 70 & 69 & 69 & 68 & 67 & 5 \\
\hline 2 & 76 & 70 & 65 & 61 & 74 & 69 & 64 & 60 & 66 & 62 & 59 & 63 & 60 & 57 & 61 & 58 & 56 & 4 \\
\hline 3 & 69 & 61 & 55 & 51 & 67 & 60 & 55 & 50 & 58 & 53 & 49 & 56 & 52 & 48 & 54 & 51 & 48 & 46 \\
\hline 4 & 63 & 54 & 48 & 43 & 62 & 53 & 47 & 42 & 51 & 46 & 42 & 50 & 45 & 41 & 48 & 44 & 41 & 9 \\
\hline 5 & 58 & 48 & 42 & 37 & 57 & 48 & 41 & 36 & 46 & 40 & 36 & 45 & 40 & 36 & 43 & 39 & 35 & 4 \\
\hline 6 & 54 & 44 & 37 & 32 & 52 & 43 & 36 & 32 & 41 & 36 & 31 & 40 & 35 & 31 & 39 & 34 & 31 & 29 \\
\hline 7 & 50 & 39 & 33 & 28 & 48 & 39 & 32 & 28 & 38 & 32 & 28 & 36 & 31 & 27 & 35 & 31 & 27 & 26 \\
\hline 8 & 46 & 36 & 29 & 25 & 45 & 35 & 29 & 25 & 34 & 29 & 25 & 33 & 28 & 24 & 32 & 28 & 24 & 23 \\
\hline 9 & 43 & 33 & 26 & 22 & 42 & 32 & 26 & 22 & 31 & 26 & 22 & 31 & 26 & 22 & 30 & 25 & 22 & 20 \\
\hline 10 & 40 & 30 & 24 & 20 & 39 & 30 & 24 & 20 & 29 & 24 & 20 & 28 & 23 & 20 & 28 & 23 & 20 & 18 \\
\hline
\end{tabular}

ALL CANDELA, LUMENS, LUMINANCE, COEFFICIENT OF UTILIZATION AND VCP VALUES IN THIS REPORT ARE BASED ON RELATIVE PHOTOMETRY WHICH ASSUMES A BALLAST FACTOR OF 1.000. ANY CALCULATIONS PREPARED FROM THESE DATA SHOULD INCLUDE AN APPROPRIATE BALLAST FACTOR. 


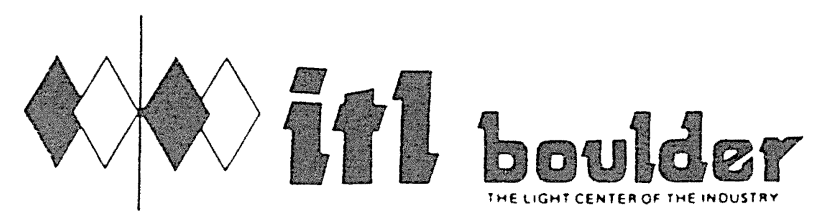

FAX (303) $449-5274$ INDEPENDENT TESTING LABORATORIES, INC. • 3386 LONGHORN ROAD, BOULDER, COLORADO 80302 • PHONE (303) 442-1255

REPORT NUMBER: ITL40780

PREPARED FOR: U.S. ARMY CORPS OF ENGINEERS

DATE: $7-29-1993$

CATALOG NUMBER: SHALLOW CAN, FULL REFLECTOR

LUMINAIRE: FABRICATED SQUARE METAI HOUSING, SPUN SPECULAR ALUMINUM REELECTOR, WHITE PAINTED METAL TRIM RING, OPEN BOTTOM.

LAMP: STANDARD GE A60 60-WATT A-19 INCANDESCENT, RATED 870 LUMENS, VERTICAL BASE-UP POSITION. MOUNTING: RECESSED

$\begin{array}{rcr}\text { DEG } & \text { CANDELA } & \text { LUMENS } \\ 0 & 930 & \\ 5 & 666 & 60 \\ 15 & 482 & 136 \\ 25 & 360 & 165 \\ 35 & 198 & 126 \\ 45 & 82 & 63 \\ 55 & 18 & 16 \\ 65 & 0 & 0 \\ 75 & 0 & 0 \\ 85 & 0 & 0 \\ 90 & 0 & \end{array}$

ZONAL LUMEN SUMMARY ZONE LUMENS

$0-30$
$0-40$
$0-60$
$0-90$
$90-180$
$0-180$

361
487
566
566
0
566

OLAMP

41.5
56.0

65.0

65.1

0.0

65.1

TOTAL LUMINAIRE EFFICIENCY = CIE TYPE - DIRECT

LUMINAIRE SPACING CRITERION $=0.5$

LUMINOUS DIAMETER: $\quad 5.875$

LUMINANCE DATA IN CANDELA/SQ M ANGLE AVERAGE MAXIMUM MAX/AVG IN DEG

$\begin{array}{rrrr}45 & 6628 . & 41710 . & 6.3 \\ 55 & 1794 . & 29181 . & 16.3 \\ 65 & 0 . & 40 . & 0.0 \\ 75 & 0 . & 21 . & 0.0 \\ 85 & 0 . & 6 . & 0.0\end{array}$
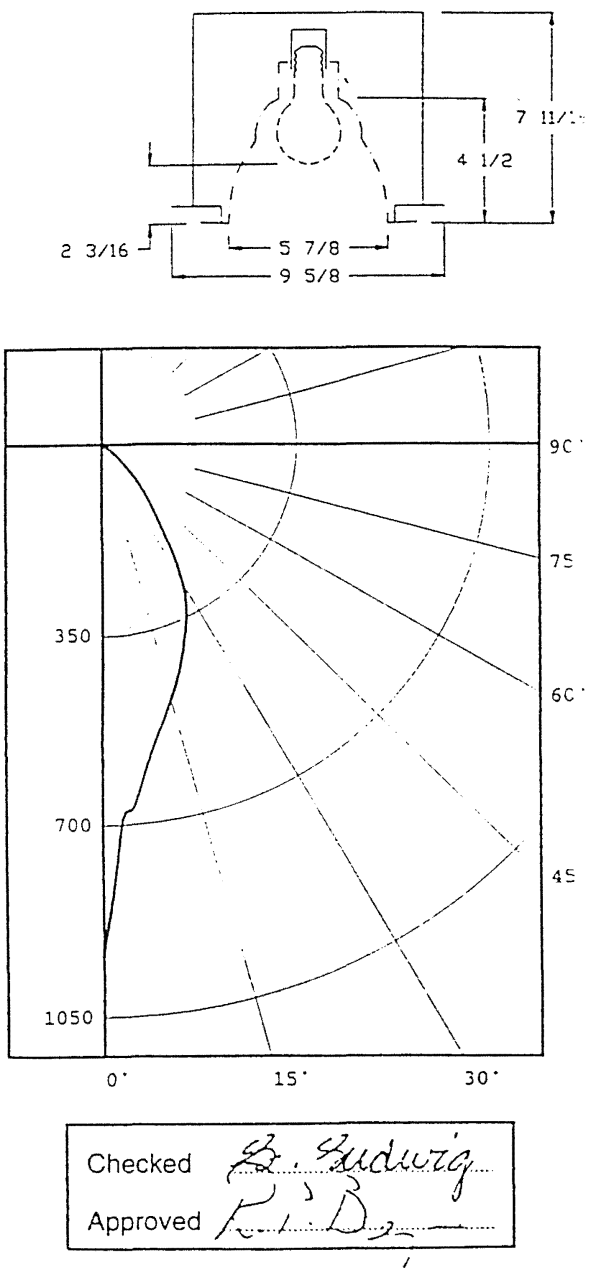


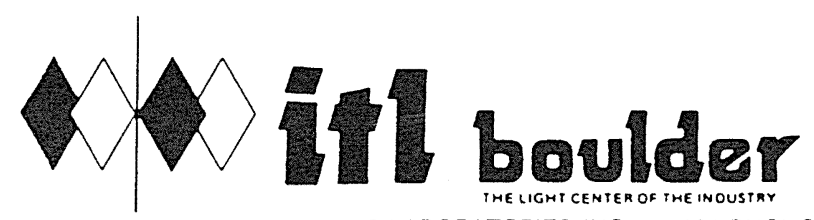

FAX (303) 449-52iINDEPENDENT TESTING LABORATORIES, INC. • 3386 LONGHORN ROAD, BOULDER, COLORADO 80302 • PHONE (303) 442-125:

REPORT NUMBER: ITL40780

DATE: $7-29-1993$

PREPARED FOR: U.S. ARMY CORPS OF ENGINEERS

\begin{tabular}{|c|c|c|c|}
\hline CANDELA & $\begin{array}{l}\text { DISTRIBUTION } \\
0.0\end{array}$ & ZONAL LUMEN & SUMMARY \\
\hline 0.0 & 930 & $0-5$ & 17 . \\
\hline 2.5 & 720 & $5-10$ & 43. \\
\hline 5.0 & 666 & $10-15$ & 61. \\
\hline 7.5 & 602 & $15-20$ & 74 \\
\hline 10.0 & 554 & $20-25$ & 81 \\
\hline 12.5 & 516 & $25-30$ & 84 \\
\hline 15.0 & 482 & $30-35$ & 73 \\
\hline 17.5 & 451 & $35-40$ & 53. \\
\hline 20.0 & 419 & $40-45$ & 39. \\
\hline 22.5 & 388 & $45-50$ & 24. \\
\hline 25.0 & 360 & $50-55$ & 13. \\
\hline 27.5 & 330 & $55-60$ & 3 \\
\hline 30.0 & 293 & $60-65$ & 0 \\
\hline 32.5 & 247 & $65-70$ & 0 \\
\hline 35.0 & 198 & $70-75$ & 0 \\
\hline 37.5 & 160 & $75-80$ & 0 \\
\hline 40.0 & 131 & $80-85$ & 0 \\
\hline 42.5 & 105 & $85-90$ & 0 . \\
\hline
\end{tabular}




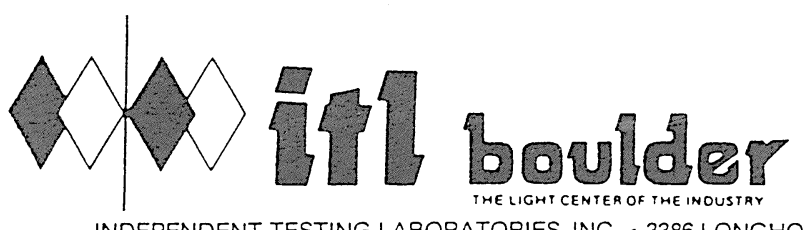

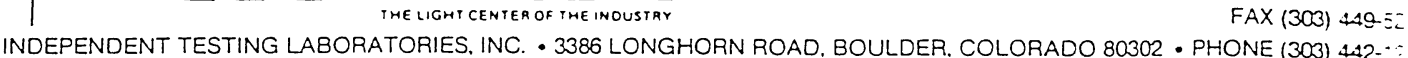

REPORT NUMBER: ITL4 0780

DATE: $7-29-1993$

PREPARED FOR: U.S. ARMY CORPS OF ENGINEERS

COEFFICIENTS OF UTILIZATION - ZONAL CAVITY METHOD

EFFECTIVE FLOOR CAVITY REFLECTANCE 0.20

\begin{tabular}{|c|c|c|c|c|c|c|c|c|c|c|c|c|c|c|c|c|c|c|}
\hline $\mathrm{RC}$ & \multicolumn{4}{|c|}{80} & \multicolumn{4}{|c|}{70} & \multicolumn{3}{|c|}{50} & \multicolumn{3}{|c|}{30} & \multicolumn{3}{|c|}{10} & 0 \\
\hline RW & 70 & 50 & 30 & 10 & 70 & 50 & 30 & 10 & 50 & 30 & 10 & 50 & 30 & 10 & 50 & 30 & 10 & 0 \\
\hline 0 & 77 & 77 & 77 & 77 & 76 & 76 & 76 & 76 & 72 & 72 & 72 & 69 & 69 & 69 & 66 & 66 & 66 & 65 \\
\hline 1 & 74 & 72 & 70 & 69 & 72 & 70 & 69 & 68 & 68 & 67 & 65 & 65 & 64 & 64 & 63 & 62 & 62 & 61 \\
\hline 2 & 70 & 67 & 64 & 62 & 68 & 65 & 63 & 61 & 63 & 61 & 59 & 61 & 60 & 58 & 60 & 58 & 57 & 55 \\
\hline 3 & 66 & 62 & 58 & 56 & 65 & 61 & 58 & 55 & 59 & 56 & 54 & 57 & 55 & 53 & 56 & 54 & 53 & 52 \\
\hline 4 & 62 & 57 & 54 & 51 & 61 & 57 & 53 & 50 & 55 & 52 & 50 & 54 & 51 & 49 & 53 & 50 & 49 & 8 \\
\hline 5 & 59 & 53 & 49 & 46 & 58 & 53 & 49 & 46 & 52 & 48 & 46 & 50 & 48 & 45 & 49 & 47 & 45 & 44 \\
\hline 6 & 56 & 50 & 46 & 43 & 55 & 49 & 45 & 43 & 48 & 45 & 42 & 47 & 44 & 42 & 47 & 44 & 42 & 41 \\
\hline 7 & 53 & 47 & 43 & 40 & 52 & 46 & 42 & 40 & 45 & 42 & 39 & 45 & 41 & 39 & 44 & 41 & 39 & 8 \\
\hline 8 & 50 & 44 & 40 & 37 & 49 & 43 & 40 & 37 & 43 & 39 & 37 & 42 & 39 & 37 & 41 & 38 & 36 & \\
\hline 9 & 48 & 41 & 37 & 34 & 47 & 41 & 37 & 34 & 40 & 37 & 34 & 40 & 36 & 34 & 39 & 36 & 34 & \\
\hline 10 & 45 & 39 & 35 & 32 & 45 & 39 & 35 & 32 & 38 & 35 & 32 & 37 & 34 & 32 & 37 & 34 & 32 & 1 \\
\hline
\end{tabular}

ALL CANDELA, LUMENS, LUMINANCE, COEFFICIENT OF UTILIZAT_ON AND VCD VALUES IN THIS REPORT ARE BASED ON RELATIVE PHOTOMETRY WHICH ASSUMES A BALLAST FACTOR OF 1.000. ANY CALCULATIONS PREPARED FROM THESE DATA SHOULD INCLUDE AN APPROPRIATE BALLAST FACTOR. 


\section{ifl boulder}

FAX (303) $449-52 i$ INDEPENDENT TESTING LABORATORIES, INC. - 3386 LONGHORN ROAD, BOULDER, COLORADO 80302 • PHONE (303) 442-125E

REPORT NUMBER: ITL40781

PREPARED FOR: U.S. ARMY CORPS OF ENGINEERS

DATE: $7-22-1993$

CATALOG NUMBER: DEEP CAN, FULL REFLECTOR

LUMINAIRE: FABRICATED CYLINDRICAL METAL HOUSING WITH BLACK PAINTED INTERIOR, SPUN SPECULAR ALUMINUM REFLECTOR, WHITE PAINTED METAL TRIM RING, OPEN BOTTOM.

LAMP: 15-WATT FLB15/TL COMPACT FLUORESCENT, RATED 70O LUMENS, VERTICAL BASE-UP POSITION.

MOUNTING: RECESSED

TOTAL INPUT WATTS $=16.1 \mathrm{AT} 120.0$ VOLTS

CANDEIAA DISTRIBUTION

$\begin{array}{rrrrrr} & 0.0 & 45.0 & 90.0 & 135.0 & 180.0 \\ 0 & 138 & 138 & 138 & 138 & 138 \\ 5 & 151 & 147 & 140 & 134 & 132 \\ 15 & 195 & 175 & 148 & 140 & 142 \\ 25 & 220 & 191 & 157 & 152 & 158 \\ 35 & 204 & 179 & 150 & 147 & 154 \\ 45 & 162 & 146 & 130 & 130 & 136 \\ 55 & 111 & 103 & 95 & 96 & 102 \\ 65 & 58 & 57 & 53 & 55 & 59 \\ 75 & 6 & 7 & 8 & 8 & 9 \\ 85 & 0 & 0 & 0 & 0 & 0 \\ 90 & 0 & 0 & 0 & 0 & 0\end{array}$

ZONAL LUMEN SUMMARY

$\begin{array}{cr}\text { ZONE } & \text { LUMENS } \\ 0-30 & 138 \\ 0-40 & 241 \\ 0-60 & 437 \\ 0-90 & 503 \\ 90-180 & 0 \\ 0-180 & 503\end{array}$

8LAMP
19.7
34.4
62.4
71.8
0.0
71.8

FLUX

14

45

79

103

107

89

55

11

0

$0-180$

503

71.8

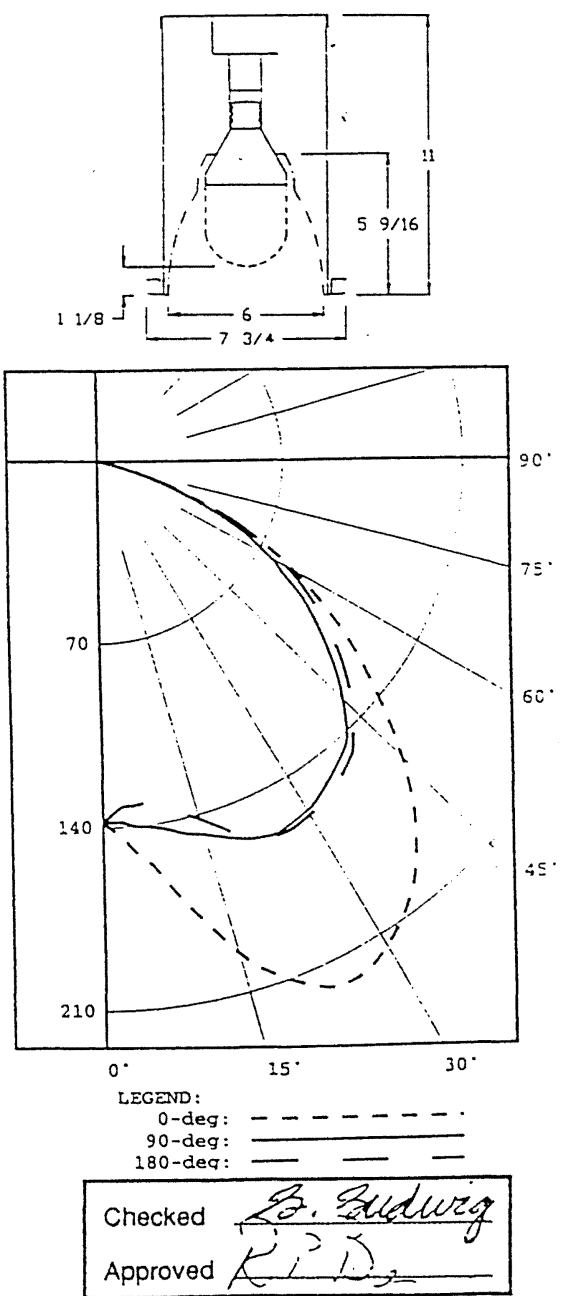

TOTAL LUMINAIRE EFFICIENCY = CIE TYPE - DIRECT
PLANE : $0-D E G$
SPACING CRITERIA : $1.8 \quad 1.6 \quad 1.6$
LUMINOUS DIAMETER: 6.000

90-DEG 180-DEG

LUMINANCE DATA IN CANDELA/SQ M

ANGLE AVERAGE AVERAGE 'AVERAGE

IN DEG 0-DEG 90-DEG 180-DEG

45 12555. 10075. 10540.

55 10605. 9076. 9745.

65 7521. 6872. 7650

75 1270. 1694. 1906.

8500.00.

ANGLE MAXIMUM MAXIMUNi MAXIMUM MAX/AVG MAX/AVG MAX/AVG

IN DEG 0-DEG 90-DEG 180-DEG 0-DEG 90-DEG 180-DEG

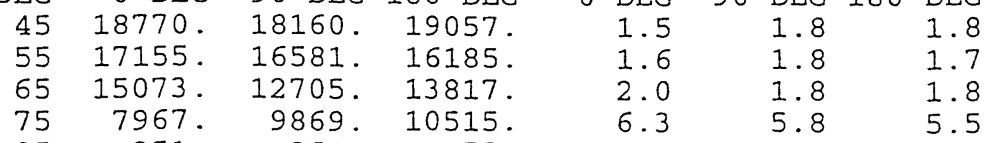

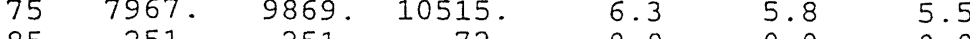

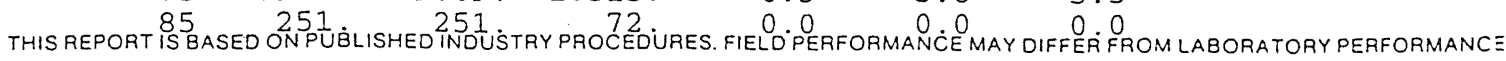




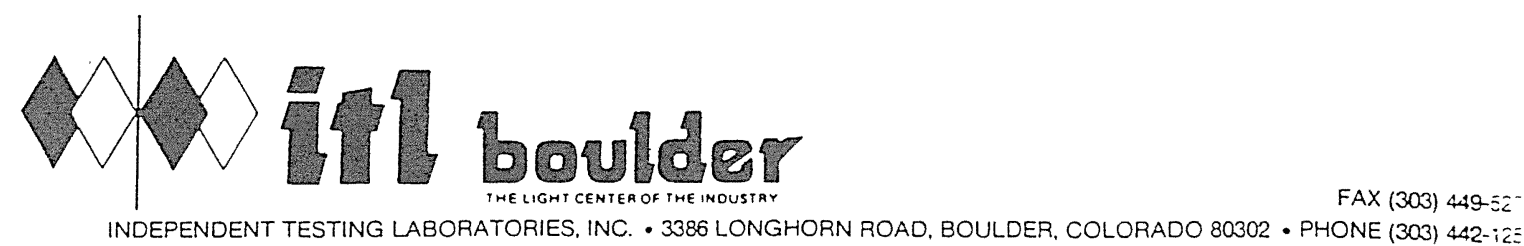

REPORT NUMBER: ITL40781

PREPARED FOR: U.S. ARMY CORPS OF ENGINEERS

DATE: $7-22-1993$

CANDELA DISTRIBUTION

$\begin{array}{rrrrrrrrrr} & 1.0 & 22.5 & 45.0 & 67.5 & 90.0 & 112.5 & 135.0 & 157.5 & 180.0 \\ 2.0 & 138 & 138 & 138 & 138 & 138 & 138 & 138 & 138 & 138 \\ 5.0 & 143 & 143 & 142 & 141 & 138 & 137 & 135 & 133 & 133 \\ 7.5 & 158 & 150 & 147 & 144 & 140 & 136 & 134 & 133 & 132 \\ 10.0 & 170 & 158 & 153 & 147 & 141 & 137 & 134 & 133 & 132 \\ 12.5 & 182 & 178 & 160 & 151 & 143 & 138 & 135 & 135 & 135 \\ 15.0 & 195 & 189 & 175 & 155 & 146 & 140 & 138 & 138 & 138 \\ 17.5 & 205 & 198 & 182 & 164 & 151 & 142 & 140 & 141 & 142 \\ 20.0 & 212 & 206 & 187 & 167 & 154 & 148 & 148 & 146 & 147 \\ 22.5 & 218 & 210 & 190 & 169 & 156 & 150 & 151 & 155 & 153 \\ 25.0 & 220 & 212 & 191 & 170 & 157 & 151 & 152 & 156 & 158 \\ 27.5 & 219 & 211 & 190 & 169 & 156 & 151 & 153 & 156 & 158 \\ 30.0 & 215 & 208 & 187 & 166 & 155 & 150 & 151 & 156 & 157 \\ 32.5 & 210 & 204 & 183 & 163 & 153 & 148 & 150 & 154 & 156 \\ 35.0 & 204 & 199 & 179 & 160 & 150 & 146 & 147 & 153 & 154 \\ 37.5 & 196 & 192 & 173 & 156 & 147 & 143 & 145 & 150 & 151 \\ 40.0 & 186 & 184 & 167 & 151 & 144 & 140 & 141 & 147 & 148 \\ 42.5 & 175 & 173 & 157 & 144 & 138 & 135 & 136 & 142 & 142 \\ 45.0 & 162 & 160 & 146 & 134 & 130 & 128 & 130 & 135 & 136 \\ 47.5 & 149 & 148 & 135 & 124 & 122 & 120 & 122 & 128 & 128 \\ 50.0 & 136 & 135 & 124 & 115 & 113 & 112 & 114 & 119 & 120 \\ 52.5 & 124 & 123 & 113 & 105 & 104 & 103 & 105 & 110 & 111 \\ 55.0 & 111 & 111 & 103 & 95 & 95 & 94 & 96 & 101 & 102 \\ 57.5 & 98 & 98 & 92 & 85 & 84 & 84 & 87 & 91 & 92 \\ 60.0 & 85 & 86 & 81 & 75 & 75 & 74 & 77 & 81 & 82 \\ 62.5 & 72 & 73 & 70 & 65 & 64 & 64 & 66 & 70 & 70 \\ 65.0 & 58 & 59 & 57 & 54 & 53 & 53 & 55 & 58 & 59 \\ 67.5 & 45 & 46 & 45 & 42 & 41 & 42 & 43 & 45 & 46 \\ 70.0 & 31 & 32 & 32 & 31 & 30 & 30 & 31 & 33 & 34 \\ 72.5 & 18 & 19 & 199 & 19 & 18 & 18 & 19 & 19 & 20 \\ 75.0 & 6 & 7 & 7 & 7 & 8 & 8 & 8 & 8 & 9 \\ 77.5 & 1 & 11 & 1 & 2 & 2 & 2 & 2 & 2 & 2 \\ 80.0 & 0 & 0 & 0 & 0 & 0 & 0 & 0 & 0 & 0 \\ 82.5 & 0 & 0 & 0 & 0 & 0 & 0 & 0 & 0 & 0 \\ 85.0 & 0 & 0 & 0 & 0 & 0 & 0 & 0 & 0 & 0 \\ 87.5 & 0 & 0 & 0 & 0 & 0 & 0 & 0 & 0 & 0 \\ 90.0 & 0 & 0 & 0 & 0 & 0 & 0 & 0 & 0 & 0\end{array}$




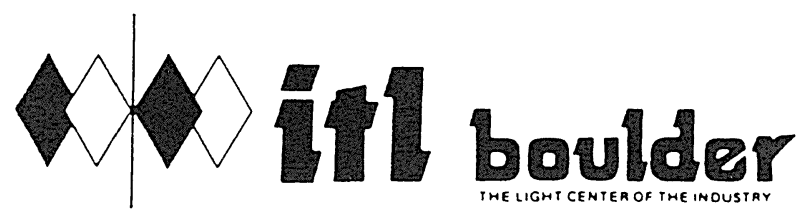

$\operatorname{FAX}(303) 4495 z$ INDEPENDENT TESTING LABORATORIES, INC. • 3386 LONGHORN ROAD, BOULDER, COLORADO 80302 • PHONE (303) $442-12$

REPORT NUMBER: ITL40781

PREPARED FOR: U.S. ARMY CORPS OF ENGINEERS

DATE: $7-22-1993$

$\begin{array}{rr}\text { ZONAL LUMEN } & \text { SUMMARY } \\ 0- & 5 \\ 5-10 & 3 . \\ 10-15 & 10 . \\ 15-20 & 18 . \\ 20-25 & 27 . \\ 25-30 & 36 . \\ 30-35 & 43 . \\ 35-40 & 49 . \\ 40-45 & 53 . \\ 45-50 & 55 . \\ 50-55 & 52 . \\ 55-60 & 48 . \\ 60-65 & 41 . \\ 65-70 & 33 . \\ 70-75 & 22 . \\ 75-80 & 10 . \\ 80-85 & 1 . \\ 85-90 & 0 .\end{array}$




\section{- It boulder}

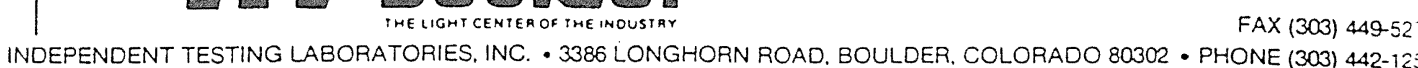

REPORT NUMBER: ITL40781

DATE : $7-22-1993$

PREPARED FOR: U.S. ARMY CORPS OF ENGINEERS

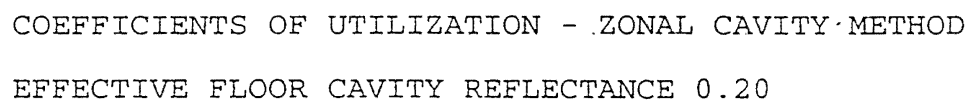

\begin{tabular}{|c|c|c|c|c|c|c|c|c|c|c|c|c|c|c|c|c|c|}
\hline & \multicolumn{3}{|l|}{80} & \multicolumn{4}{|c|}{70} & \multicolumn{3}{|c|}{50} & \multicolumn{3}{|c|}{30} & \multicolumn{3}{|c|}{10} & \\
\hline 70 & 50 & 30 & 10 & 70 & 50 & 30 & 10 & 50 & 30 & 10 & 50 & 30 & 10 & 50 & 30 & 10 & 0 \\
\hline 86 & 686 & 86 & 86 & 84 & 84 & 84 & 84 & 80 & 80 & 8 & 6 & 76 & 76 & 73 & 73 & 73 & \\
\hline 79 & 76 & 74 & 71 & 77 & 75 & 72 & 7 & 2 & 70 & 6 & 89 & 67 & 6 & 6 & 65 & & \\
\hline 73 & 67 & 63 & 59 & 71 & 66 & 62 & 5 & 3 & 60 & 5 & 61 & 58 & 5 & 9 & 57 & & \\
\hline 66 & 659 & 54 & 49 & 65 & 58 & 53 & 49 & 56 & 52 & 4 & 54 & 50 & 4 & 2 & 49 & 4 & \\
\hline & 53 & 47 & 42 & 9 & 52 & 46 & 4 & 50 & 4 & 4 & 48 & 44 & 4 & 7 & 43 & & \\
\hline 56 & 47 & 41 & 36 & 54 & 46 & 40 & 36 & 45 & 39 & 3 & 43 & 39 & 3 & 42 & 38 & 3 & \\
\hline & 42 & 36 & 3 & U & 41 & 35 & 3 & 40 & 35 & 3 & 39 & 34 & 3 & 8 & 34 & 3 & \\
\hline & 38 & 32 & 27 & 46 & 37 & 32 & 2 & & 31 & & & 31 & 2 & 4 & 3 & & \\
\hline & 0 & 29 & 24 & 43 & 34 & 2 & 24 & 33 & 28 & 2 & 32 & 27 & 24 & 31 & 27 & 2 & \\
\hline 4 & 32 & $2 \epsilon$ & 22 & 40 & 3 & 2 & 22 & U & 25 & 2 & 30 & 25 & 21 & 0 & 2 & 21 & \\
\hline & & & 0 & 38 & 29 & 23 & 20 & 28 & 23 & 19 & 27 & 23 & 19 & 27 & 22 & 19 & \\
\hline
\end{tabular}

ALL CANDELA, LUMENS, LUMINANCE, COEFFICIENT OF UTILIZATION AND VCP VALUES IN THIS REPORT ARE BASED ON RELATIVE PHOTOMETRY WHICH ASSUMES A BALLAST FACTOR OF 1.000. ANY CALCULATIONS PREPARED FROM THESE DATA SHOULD INCLUDE AN APPROPRIATE BALLAST FACTOR. 


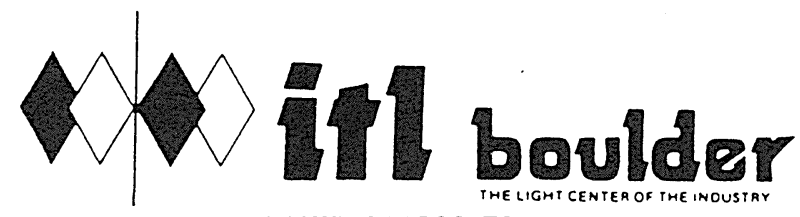

FAX (303) 449-527: INDEPENDENT TESTING LABORATORIES, INC. • 3386 LONGHORN ROAD, BOULDER, COLORADO 80302 • PHONE (303) 442-1255 REPORT NUMBER: ITL40782 PREPARED FOR: U.S. ARMY CORPS OF ENGINEERS CATALOG NUMBER: DEEP CAN, FULL REFLECTOR

LUMINAIRE: FABRICATED CYLINDRICAL METAL HOUSING WITH BLACK PAINTED INTERIOR, SPUN SPECULAR ALUMINUM REFLECTOR, WHITE PAINTED METAL TRIM RING, OPEN BOTTOM.

LAMP: 11-WATT OSRAM DULUX EL COMPACT FLUORESCENT, RATED 600 LUMENS, VERTICAL BASE-UP POSITION. MOUNTING : RECESSED

TOTAL INPUT WATTS $=11.6 \mathrm{AT} 120.0$ VOLTS

CANDELA DISTRIBUTION

$\begin{array}{rrrrrr} & 0.0 & 22.5 & 45.0 & 67.5 & 90.0 \\ 0 & 39 & 39 & 39 & 39 & 39 \\ 5 & 41 & 40 & 40 & 40 & 40 \\ 15 & 44 & 45 & 46 & 45 & 45 \\ 25 & 57 & 59 & 61 & 60 & 59 \\ 35 & 86 & 90 & 92 & 93 & 89 \\ 45 & 126 & 132 & 129 & 132 & 129 \\ 55 & 123 & 124 & 118 & 121 & 124 \\ 65 & 83 & 81 & 76 & 79 & 84 \\ 75 & 22 & 23 & 24 & 26 & 28 \\ 85 & 0 & 0 & 0 & 0 & 0 \\ 90 & 0 & 0 & 0 & 0 & 0\end{array}$

ZONAL LUMEN SUMMARY

$\begin{array}{cr}\text { ZONE } & \text { LUMENS } \\ 0-30 & 45 \\ 0-40 & 103 \\ 0-60 & 311 \\ 0-90 & 417 \\ 90-180 & 0 \\ 0-180 & 417\end{array}$

8 LAMP
7.5
17.1
51.8
69.5
0.0
69.5

FLUX

0.0
39

$40 \quad 4$

$45 \quad 13$

$59 \quad 28$

$89 \quad 58$

$129 \quad 100$

$124 \quad 108$

$\begin{array}{ll}84 & 79 \\ 28 & 27\end{array}$

$0 \quad 0$

DATE : 7-22-1993

0

TOTAL LUMINAIRE EFFICIENCY = CIE TYPE - DIRECT

$\begin{array}{lccc}\text { PLANE } & 0-\text { DEG } & 90-D E G \\ \text { SPACING CRITERIA : } & 3.0 & 3.0 \\ \text { LUMINOUS DIAMETER: } & 6.000 & \end{array}$

LUMINANCE DATA IN,CANDELA/SQ M ANGLE AVERAGE AVÉRAGE IAVERAGE IN DEG 0-DEG 45-DEG 90-DEG 459734.9966 .9028$. 55 11761. 11245. 11857. 65 10698. 9907. 10918. 75 4658. 5103. 6013. 85126.189 .189$.

ANGLE MAXIMUM MAXIMIM MAX/AVG MAX/AVG

IN DEG 0-DEG 90-DEG 0-DEG 90-DEG

45 38965. 35521. 4.0 3.5

55 40525. 41923 . $3.4 \quad 3.5$

65 40850. 40005. $3.8 \quad 3.7$

7524796 . 25641. 5.3 . 4.3
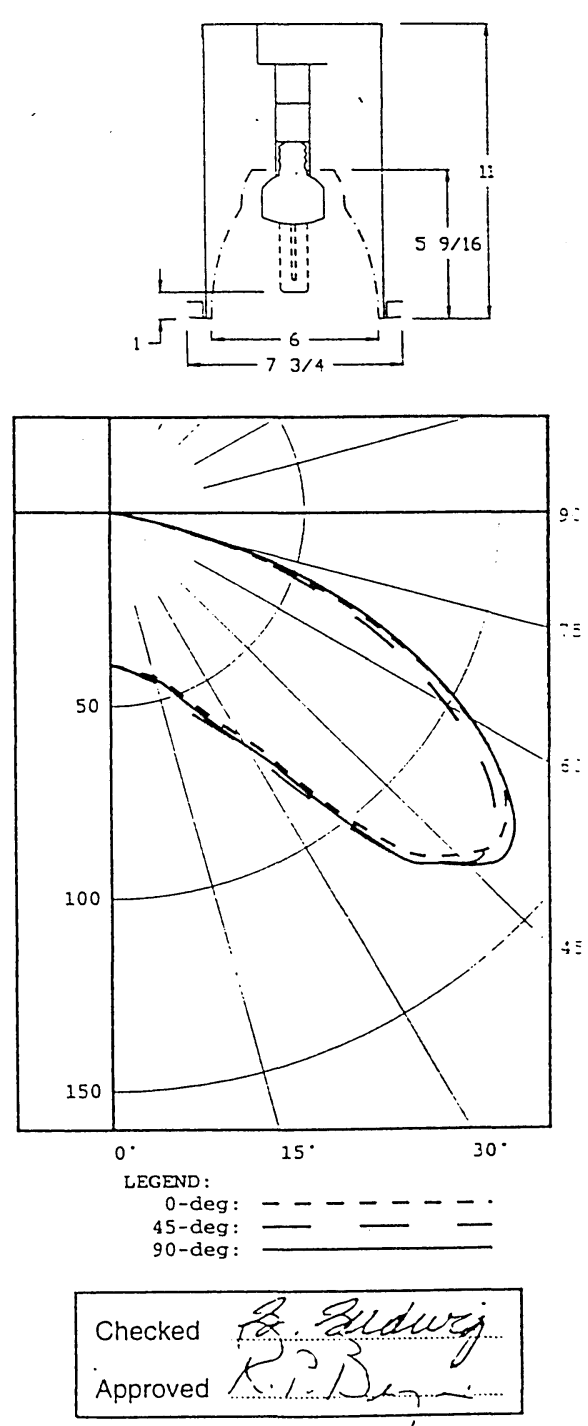

THIS REPORT IS BASED ON PUBLISHED INDUSTTRY PROCEEDURES. FIELD P PERFORMANCE MAY DIFFER FROM LABORATORY PERFCRMANC 


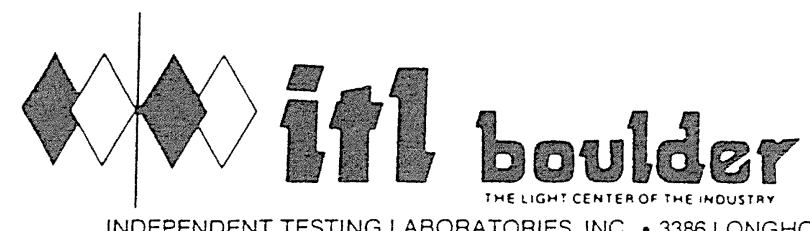

FAX (303) 440-52:= INDEPENDENT TESTING LABORATORIES, INC. • 3386 LONGHORN ROAD, BOULDER. COLORADO 80302 • PHONE (303) 442-12E3

REPORT NUMBER: ITL40782

PREPARED FOR: U.S. ARMY CORPS OF ENGINEERS

DATE: $7-22-1993$

CANDELA DISTRIBUTION

\begin{tabular}{|c|c|c|c|c|c|c|c|c|}
\hline & 0.0 & 22.5 & 45.0 & 67.5 & 90.0 & ZONAL & LUMEN & SUMMARY \\
\hline 0.0 & 39 & 39 & 39 & 39 & 39 & $0-$ & 5 & 1. \\
\hline 2.5 & 40 & 40 & 40 & 40 & 40 & $5-1$ & 10 & 3 \\
\hline 5.0 & 41 & 40 & 40 & 40 & 40 & $10-1$ & 15 & 5 \\
\hline 7.5 & 41 & 41 & 42 & 42 & 42 & $15-2$ & 20 & 8 \\
\hline 10.0 & 42 & 42 & 43 & 43 & 42 & $20-2$ & 25 & 12 \\
\hline 12.5 & 43 & 43 & 44 & 44 & 43 & $25-3$ & 30 & 16 \\
\hline 15.0 & 44 & 45 & 46 & 45 & 45 & $30-3$ & 35 & 23 \\
\hline 17.5 & 46 & 47 & 49 & 48 & 47 & $35-$ & 40 & 35 \\
\hline 20.0 & 49 & 51 & 53 & 52 & 50 & $40-4$ & 45 & 46 \\
\hline 22.5 & 52 & 55 & 57 & 56 & 54 & $45-5$ & 50 & 54 \\
\hline 25.0 & 57 & 59 & 61 & 60 & 59 & $50-5$ & 55 & 56 \\
\hline 27.5 & 62 & 64 & 65 & 66 & 64 & $55-6$ & 60 & 52 \\
\hline 30.0 & 67 & 70 & 71 & 71 & 70 & $60-6$ & 65 & 45 \\
\hline 32.5 & 74 & 78 & 81 & 81 & 77 & $65-7$ & 70 & 34 \\
\hline 35.0 & 86 & 90 & 92 & 93 & 89 & $70-7$ & 75 & 21 \\
\hline 37.5 & 99 & 104 & 105 & 107 & 103 & $75-8$ & 80 & 6 \\
\hline 40.0 & 113 & 117 & 117 & 120 & 118 & $80-8$ & 85 & 0 \\
\hline 42.5 & 121 & 125 & 123 & 127 & 123 & $85-5$ & 90 & \\
\hline 45.0 & 126 & 132 & 129 & 132 & 129 & & & \\
\hline 47.5 & 130 & 136 & 130 & .134 & 134 & & & \\
\hline 50.0 & 131 & 135 & 129 & 133 & 134 & & & \\
\hline 52.5 & 128 & 131 & 124 & 128 & 131 & & & \\
\hline 55.0 & 123 & 124 & 118 & 121 & 124 & & & \\
\hline 57.5 & 116 & 116 & 109 & 113 & 116 & & & \\
\hline 60.0 & 106 & 105 & 99 & 103 & 107 & & & \\
\hline 62.5 & 95 & 94 & 89 & 92 & 96 & & & \\
\hline 65.0 & 83 & 81 & 76 & 79 & 84 & & & \\
\hline 67.5 & 69 & 68 & 64 & 67 & 72 & & & \\
\hline 70.0 & 56 & 54 & 52 & 55 & 60 & & & \\
\hline 72.5 & 41 & 40 & 39 & 42 & 45 & & & \\
\hline 75.0 & 22 & 23 & 24 & 26 & 28 & & & \\
\hline 77.5 & 9 & 10 & 10 & 12 & 13 & & & \\
\hline 80.0 & 1 & 1 & 2 & 2 & 2 & & & \\
\hline 82.5 & 0 & 1 & 1 & 1 & 1 & & & \\
\hline 85.0 & 0 & 0 & $\sigma$ & 0 & 0 & & & \\
\hline 87.5 & 0 & 0 & 0 & 0 & 0 & & & \\
\hline 90.0 & 0 & 0 & 0 & 0 & 0 & & & \\
\hline
\end{tabular}




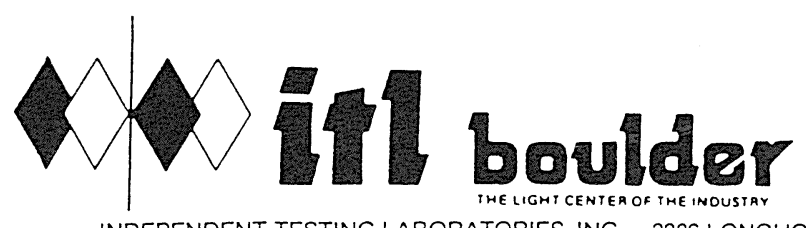

INDEPENDENT TESTING LABORATORIES, INC • 3386 LONGHORN ROAD, BOULDER, COLORADO 80302 • PI

FAX (303) 449-52:

REPORT NUMBER: ITL 40782

DATE : $7-22-1993$

PREPARED FOR: U.S. ARMY CORPS OF ENGINEERS

COEFFICIENTS OF UTILIZATION - ZONAL CAVITY METHOD

EFFECTIVE FLOOR CAVITY REFLECTANCE 0.20

\begin{tabular}{|c|c|c|c|c|c|c|c|c|c|c|c|c|c|c|c|c|c|}
\hline $\mathrm{RC}$ & \multicolumn{3}{|c|}{80} & \multicolumn{4}{|c|}{70} & \multicolumn{3}{|c|}{50} & \multicolumn{3}{|c|}{30} & \multicolumn{3}{|c|}{10} & 0 \\
\hline $\mathrm{RW}$ & $70 \quad 50$ & 30 & 10 & 70 & 50 & 30 & 10 & 50 & 30 & 10 & 50 & 30 & 10 & 50 & 30 & 10 & 0 \\
\hline 0 & 8383 & 83 & 83 & 81 & 81 & 81 & 81 & 77 & 77 & 77 & 74 & 74 & 74 & 71 & 71 & 71 & 70 \\
\hline 1 & $75 \quad 72$ & 68 & 66 & 73 & 70 & 67 & 65 & 67 & 65 & 62 & 64 & 62 & 61 & 62 & 60 & 59 & 57 \\
\hline 2 & $67 \quad 61$ & 56 & 51 & 65 & 59 & 55 & 51 & 57 & 53 & 49 & 55 & 51 & 48 & 52 & 50 & 47 & 45 \\
\hline 3 & $60 \quad 52$ & 45 & 40 & 58 & 51 & 45 & 40 & 48 & 43 & 39 & 46 & 42 & 38 & 45 & 41 & 38 & 36 \\
\hline 4 & $\begin{array}{lll}54 & 44\end{array}$ & 38 & 32 & 52 & 43 & 37 & 32 & 42 & 36 & 32 & 40 & 35 & 31 & 38 & 34 & 31 & 29 \\
\hline 5 & 4939 & 31 & 26 & 47 & 38 & 31 & 26 & 36 & 30 & 26 & 35 & 29 & 25 & 33 & 29 & 25 & 23 \\
\hline 6 & $44 \quad 34$ & 27 & 22 & 43 & 33 & 26 & 22 & 32 & 26 & 21 & 30 & 25 & 21 & 29 & 25 & 21 & 19 \\
\hline 7 & 4030 & 23 & 18 & 39 & 29 & 23 & 18 & 28 & 22 & 18 & 27 & 22 & 18 & 26 & 21 & 18 & 16 \\
\hline 8 & 3727 & 20 & 15 & 36 & 26 & 20 & 15 & 25 & 19 & 15 & 24 & 19 & 15 & 23 & 18 & 15 & 13 \\
\hline 9 & 3424 & 17 & 13 & 33 & 23 & 17 & 13 & 23 & 17 & 13 & 22 & 17 & 13 & 21 & 16 & 13 & 11 \\
\hline 10 & $32 \quad 22$ & 16 & 11 & 31 & 21 & 15 & 11 & 20 & 15 & 11 & 20 & 15 & 11 & 19 & 15 & 11 & 10 \\
\hline
\end{tabular}

ALL CANDELA, LUMENS, LUMINANCE, COEFFICIENT OF UTILIZATION AND VCP VALUES IN THIS REPORT ARE BASED ON RELATIVE PHOTOMETRY WHICH ASSUMES A BALLAST FACTOR OF 1.000. ANY CALCULATIONS PREPARED FROM THESE DATA SHOULD INCLUDE AN APPROPRIATE BALLAST FACTOR. 


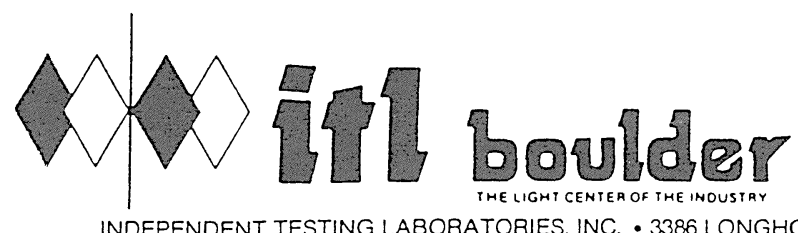

INDEPENDENT TESTING LABORATORIES, INC. • 3386 LONGHORN ROAD. BOULDER. COLORADO 80302 • PHONE (303) 442-1255

REPORT NUMBER: ITL40783

PREPARED FOR: U.S. ARMY CORPS OF ENGINEERS

DATE : 7-27-1993

CATALOG NUMBER: DEEP CAN, FULL REFLECTOR

LUMINAIRE: FABRICATED CYLINDRICAI METAL HOUSING WITH BLACK PAINTED INTERIOR, SPUN SPECULAR ALUMINUM REFLECTOR, WHITE PAINTED METAL TRIM RING, OPEN BOTTOM.

LAMP: 13-WATT LUMATECH RAS-130 REFLECT-A-STAR WITH 13-WATT QUAD TUBE FLUORESCENT, RATED 860 LUMENS, VERTICAL BASE-UP POSITION.

MOUNTING : RECESSED

TOTAL INPUT WATTS $=15.7$ AT 120.0 VOLTS

$\begin{array}{rrr}\text { DEG } & \text { CANDELA } & \text { LUMENS } \\ 0 & 232 & \\ 5 & 230 & 22 \\ 15 & 195 & 55 \\ 25 & 143 & 66 \\ 35 & 107 & 67 \\ 45 & 81 & 63 \\ 55 & 61 & 54 \\ 65 & 39 & 39 \\ 75 & 15 & 16 \\ 85 & 0 & 1 \\ 90 & 0 & \end{array}$

ZONAL LUMEN SUMMARY ZONE

$0-30$

LUMENS

$0-40$

143

$0-60$

210

$0-90$

$90-180$

327

382

$0-180$

382

$$
\begin{array}{r}
\text { 8LAMP } \\
16.6 \\
24.4 \\
38.0 \\
44.4 \\
0.0 \\
44.4
\end{array}
$$

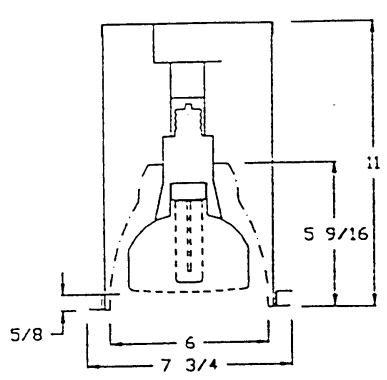

TOTAL LUMINAIRE EFFICIENCY =

CIE TYPE - DIRECT

LUMINAIRE SPACING CRITERION $=0.9$

LUMINOUS DIAMETER: 6.000

\begin{tabular}{rrrr}
\multicolumn{2}{c}{ LUMINANCE DATA } & \multicolumn{3}{c}{ IN CANDELA/SQ M } \\
ANGLE & AVERAGE & MAXIMUM MAX/AVG \\
IN DEG & 0-DEG & O-DEG & 0-DEG \\
45 & 6277. & 34067. & 5.4 \\
55 & 5828. & 29444. & 5.1 \\
65 & 5057. & 22822. & 4.5 \\
75 & 3176. & 10661. & 3.4 \\
85 & 0. & 1624. & 0.0
\end{tabular}
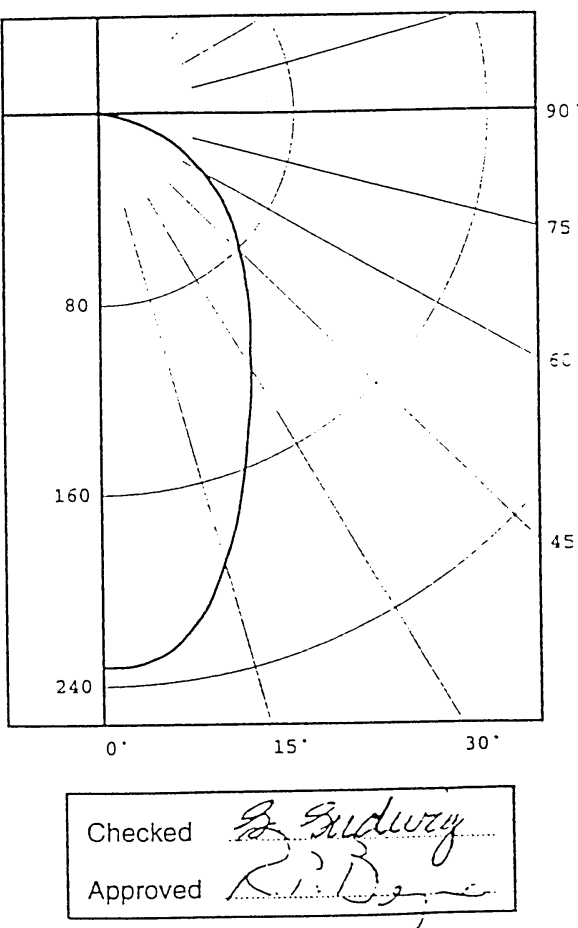


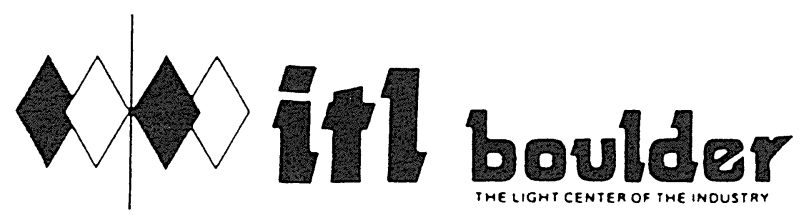

INDEPENDENT TESTING LABORATORIES, INC. • 3386 LONGHORN ROAD. BOULDER, COLORADO 80302 • PHONE (303) 442-125

REPORT NUMBER: ITL40783

PREPARED FOR: U.S. ARMY CORPS OF ENGINEERS

DATE : $7-27-1993$

\begin{tabular}{|c|c|}
\hline CANDELA & DISTRIBUTION \\
\hline & 0.0 \\
\hline 0.0 & 232 \\
\hline 2.5 & 232 \\
\hline 5.0 & 230 \\
\hline 7.5 & 226 \\
\hline 10.0 & 218 \\
\hline 12.5 & 208 \\
\hline 15.0 & 195 \\
\hline 17.5 & 182 \\
\hline 20.0 & 168 \\
\hline 22.5 & 155 \\
\hline 25.0 & 143 \\
\hline 27.5 & 133 \\
\hline 30.0 & 123 \\
\hline 32.5 & 114 \\
\hline 35.0 & 107 \\
\hline 37.5 & 100 \\
\hline 40.0 & 93 \\
\hline 42.5 & 87 \\
\hline 45.0 & 81 \\
\hline 47.5 & 76 \\
\hline 50.0 & 71 \\
\hline 52.5 & 66 \\
\hline 55.0 & 61 \\
\hline 57.5 & 55 \\
\hline 60.0 & 50 \\
\hline 62.5 & 44 \\
\hline 65.0 & 39 \\
\hline 67.5 & 34 \\
\hline 70.0 & 28 \\
\hline 72.5 & 21 \\
\hline 75.0 & 15 \\
\hline 77.5 & 9 \\
\hline 80.0 & 5 \\
\hline 82.5 & 2 \\
\hline 85.0 & 0 \\
\hline 87.5 & 0 \\
\hline 90.0 & 0 \\
\hline
\end{tabular}

$\begin{array}{rr}\text { ZONAI LUMEN SUMMARY } \\ 0-55 . \\ 5-10 & 6 . \\ 10-15 & 25 . \\ 15-20 & 30 . \\ 20-25 & 33 . \\ 25-30 & 34 . \\ 30-35 & 34 . \\ 35-40 & 33 . \\ 40-45 & 32 . \\ 45-50 & 31 . \\ 50-55 & 29 . \\ 55-60 & 25 . \\ 60-65 & 21 . \\ 65-70 & 17 . \\ 70-75 & 11 . \\ 75-80 & 5 . \\ 80-85 & 1 . \\ 85-90 & 0 .\end{array}$




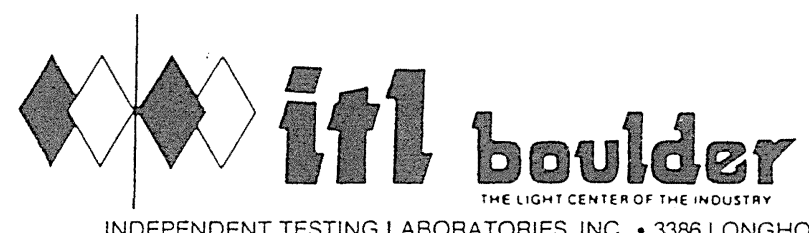

FAX (303) 449-52-

INDEPENDENT TESTING LABORATORIES. INC. • 3386 LONGHORN ROAD. BOULDER, COLORADO 80302 • PHONE (303) 442-125

REPORT NUMBER: ITL40783

DATE: $7-27-1993$

PREPARED FOR: U.S. ARMY CORPS OF ENGINEERS

COEFFICIENTS OF UTILIZATION - ZONAL CAVITY METHOD

EFFECTIVE FLOOR CAVITY REFLECTANCE 0.20

\begin{tabular}{|c|c|c|c|c|c|c|c|c|c|c|c|c|c|c|c|c|c|}
\hline$R C$ & \multicolumn{3}{|c|}{80} & \multicolumn{4}{|c|}{70} & \multicolumn{3}{|c|}{50} & \multicolumn{3}{|c|}{30} & \multicolumn{3}{|c|}{10} & 0 \\
\hline $\mathrm{RW}$ & $70 \quad 50$ & 30 & 10 & 70 & 50 & 30 & 10 & 50 & 30 & 10 & 50 & 30 & 10 & 50 & 30 & 10 & 0 \\
\hline 0 & $53 \quad 53$ & 53 & 53 & 52 & 52 & 52 & 52 & 49 & 49 & 49 & 47 & 47 & 47 & 45 & 45 & 45 & 45 \\
\hline 1 & $49 \quad 47$ & 46 & 44 & 48 & 46 & 45 & 44 & 44 & 43 & 42 & 43 & 42 & 41 & 41 & 40 & 40 & 39 \\
\hline 2 & $45 \quad 42$ & 39 & 37 & 44 & 41 & 39 & 37 & 40 & 38 & 36 & 38 & 36 & 35 & 37 & 35 & 34 & 3 \\
\hline 3 & $42 \quad 37$ & 34 & 32 & 41 & 37 & 34 & 31 & 35 & 33 & 31 & 34 & 32 & 30 & 33 & 31 & 30 & 9 \\
\hline 4 & $38 \quad 34$ & 30 & 27 & 37 & 33 & 30 & 27 & 32 & 29 & 27 & 31 & 28 & 26 & 30 & 28 & 26 & 5 \\
\hline 5 & 3630 & 27 & 24 & 35 & 30 & 26 & 24 & 29 & 26 & 24 & 28 & 26 & 23 & 27 & 25 & 23 & 2 \\
\hline 6 & 3328 & 24 & 21 & 32 & 27 & 24 & 21 & 26 & 23 & 21 & 26 & 23 & 21 & 25 & 23 & 21 & \\
\hline 7 & 3125 & 22 & 19 & 30 & 25 & 22 & 19 & 24 & 21 & 19 & 24 & 21 & 19 & 23 & 21 & 19 & \\
\hline 8 & $29 \quad 23$ & 20 & 17 & 28 & 23 & 20 & 17 & 23 & 19 & 17 & 22 & 19 & 17 & 21 & 19 & 17 & \\
\hline 0 & $27 \quad 22$ & 18 & 16 & 27 & 21 & 18 & 16 & 21 & 18 & 16 & 20 & 18 & 16 & 20 & 18 & 16 & \\
\hline 10 & 2620 & 17 & 15 & 25 & 20 & 17 & 15 & 20 & 17 & 15 & 19 & 16 & 15 & 19 & 16 & 14 & \\
\hline
\end{tabular}

ALL CANDELA, LUMENS, LUMINANCE, COEFFICIENT OF UTILIZATION AND VCP VALUES IN THIS REPORT ARE BASED ON RELATIVE PHOTOMETRY WHICH ASSUMES A BALLAST FACTOR OF 1.000. ANY CALCULATIONS PREPARED FROM THESE DATA SHOULD INCLUDE AN APPROPRIATE BALLAST FACTOR. 


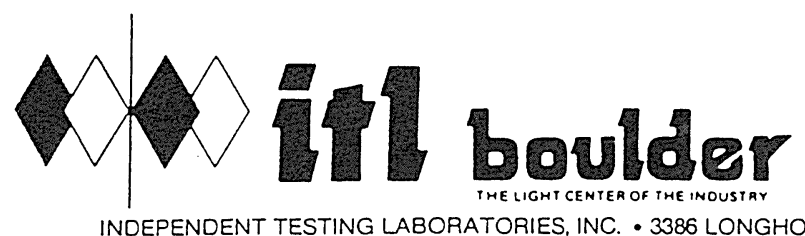

INDEPENDENT TESTING LABORATORIES, INC. • 3386 LONGHORN ROAD, BOULDER, COLORADO 80302 • PHONE (303) 442-125:

REPORT NUMBER: ITL40784

PREPARED FOR: U.S. ARMY CORPS OF ENGINEERS

DATE : 7-29-1993

CATALOG NUMBER: DEEP CAN, FULI REFLECTOR

LUMINAIRE: FABRICATED CYLINDRICAL METAL HOUSING WITH BLACK PAINTED INTERIOR, SPUN SPECULAR ALUMINUM REFLECTOR, WHITE PAINTED METAL TRIM RING, OPEN BOTTOM.

LAMP: STANDARD GE A60 60-WATT A-19 INCANDESCENT, RATED 870 LUMENS, VERTICAL BASE-UP POSITION.

MOUNTING : RECESSED

$\begin{array}{rrr}\text { DEG } & \text { CANDELA } & \text { LUMENS } \\ 0 & 924 & \\ 5 & 556 & 51 \\ 15 & 449 & 128 \\ 25 & 360 & 163 \\ 35 & 176 & 109 \\ 45 & 45 & 34 \\ 55 & 1 & 1 \\ 65 & 0 & 0 \\ 75 & 0 & 0 \\ 85 & 0 & 0 \\ 90 & 0 & \end{array}$

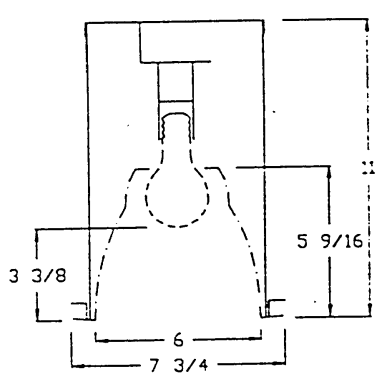

$\begin{array}{lrrr}\text { ZONAL LUMEN } & \text { SUMMARY } & & \\ \text { ZONE } & \text { LUMENS } & \text { 8LAMP } & \text { \&FIXT } \\ 0-30 & 342 & 39.3 & 70.4 \\ 0-40 & 451 & 51.8 & 92.9 \\ 0-60 & 486 & 55.8 & 100.0 \\ 0-90 & 486 & 55.8 & 100.0 \\ 90-180 & 0 & 0.0 & 0.0 \\ 0-180 & 486 & 55.8 & 100.0\end{array}$

TOTAI LUMINAIRE EFFICIENCY $=\quad 55.8 \%$ CIE TYPE - DIRECT

LUMINAIRE SPACING CRITERION $=0.4$

LUMINOUS DIAMETER: 6.000

LUMINANCE DATA IN CANDELA/SQ M ANGLE AVERAGE MAXIMUM MAX/AVG IN DEG

$\begin{array}{rrrr}45 & 3487 . & 22782 . & 6.5 \\ 55 & 96 . & 190 . & 2.0 \\ 65 & 0 . & 61 . & 0.0 \\ 75 & 0 . & 29 . & 0.0 \\ 85 & 0 . & 8 . & 0.0\end{array}$

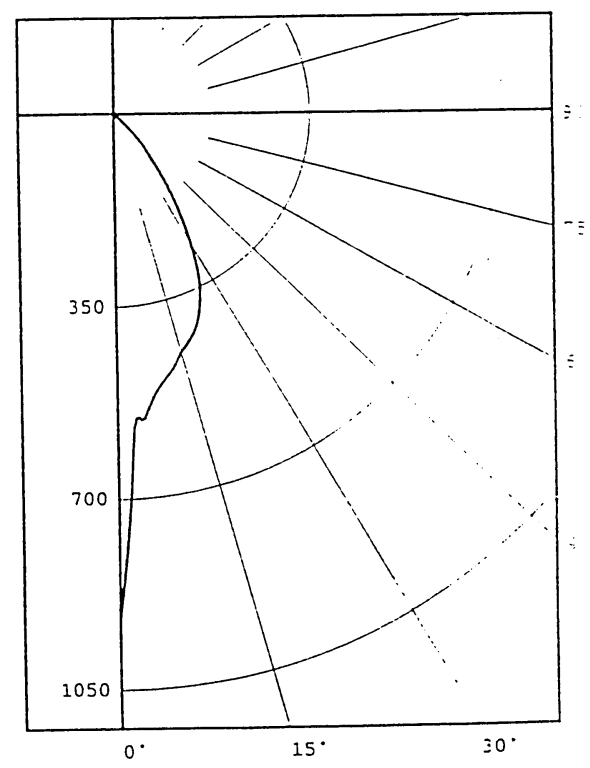

Checked

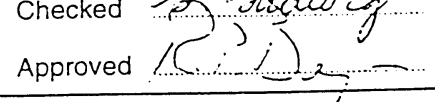

THIS REPORT IS BASED ON PUBLISHED INDUSTRY PROCEDURES. FIELD PERFORMANCE MAY DIFFER FROM LABORATORY PEAFOGMANC: 


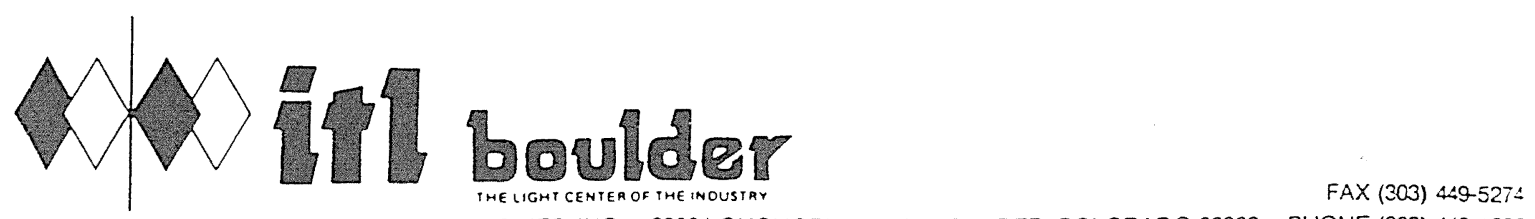
INDEPENDENT TESTING LABORATORIES, INC. • 3386 LONGHORN ROAD, BOULDER, COLORADO 80302 • PHONE (303) 442-1255

REPORT NUMBER: ITL40784

PREPARED FOR: U.S. ARMY CORPS OF ENGINEERS

DATE: $7-29-1993$

\begin{tabular}{|c|c|}
\hline CANDELA & $\begin{array}{l}\text { DISTRIBUTION } \\
0.0\end{array}$ \\
\hline 0.0 & 924 \\
\hline 2.5 & 615 \\
\hline 5.0 & 556 \\
\hline 7.5 & 514 \\
\hline 10.0 & 490 \\
\hline 12.5 & 472 \\
\hline 15.0 & 449 \\
\hline 17.5 & 434 \\
\hline 20.0 & 417 \\
\hline 22.5 & 391 \\
\hline 25.0 & 360 \\
\hline 27.5 & 320 \\
\hline 30.0 & 272 \\
\hline 32.5 & 223 \\
\hline 35.0 & 176 \\
\hline 37.5 & 130 \\
\hline 40.0 & 95 \\
\hline 42.5 & 77 \\
\hline 45.0 & 45 \\
\hline 47.5 & 13 \\
\hline 50.0 & 4 \\
\hline 52.5 & 1 \\
\hline 55.0 & 1 \\
\hline 57.5 & 1 \\
\hline 60.0 & 1 \\
\hline 62.5 & 0 \\
\hline 65.0 & 0 \\
\hline 67.5 & 0 \\
\hline 70.0 & 0 \\
\hline 72.5 & 0 \\
\hline 75.0 & 0 \\
\hline 77.5 & 0 \\
\hline 80.0 & 0 \\
\hline 82.5 & 0 \\
\hline 85.0 & 0 \\
\hline 87.5 & 0 \\
\hline 90.0 & 0 \\
\hline
\end{tabular}

ZONAL LUMEN SUMMARY

$0-515$.

$5-10 \quad 37$.

$10-15 \quad 56$.

$15-20 \quad 72$.

$20-25 \quad 82$.

$25-30 \quad 81$.

$30-35 \quad 66$.

$35-40 \quad 43$.

$40-4529$.

$45-50 \quad 5$.

$50-550$.

$55-60 \quad 0$.

$60-650$.

$65-70 \quad 0$.

$70-750$.

$75-80 \quad 0$.

$\begin{array}{ll}80-85 & 0 . \\ 85-90 & 0 .\end{array}$ 


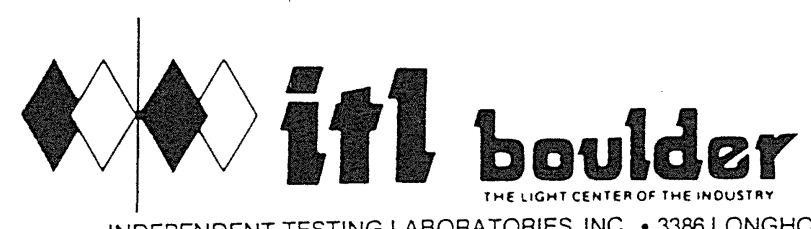

INDEPENDENT TESTING LABORATORIES, INC. • 3386 LONGHORN ROAD, BOULDER, COLORADO 80302 • PHONE (303) 442-1द

PREPARED FOR: U.S. ARMY CORPS OF ENGINEERS

\author{
COEFFICIENTS OF UTILIZATION - ZONAL CAVITY METHOD \\ EFFECTIVE FLOOR CAVITY REFLECTANCE 0.20
}

\begin{tabular}{|c|c|c|c|c|c|c|c|c|c|c|c|c|c|c|c|c|c|}
\hline $\mathrm{RC}$ & \multicolumn{3}{|c|}{80} & \multicolumn{4}{|c|}{70} & \multicolumn{3}{|c|}{50} & \multicolumn{3}{|c|}{30} & \multicolumn{3}{|c|}{10} & 0 \\
\hline$W$ & $70 \quad 50$ & 30 & 10 & 70 & 50 & 30 & 10 & 50 & 30 & 10 & 50 & 30 & 10 & 50 & 30 & 10 & 0 \\
\hline 0 & $67 \quad 67$ & 67 & 67 & 65 & 65 & 65 & 65 & 62 & 62 & 62 & 59 & 59 & 59 & 57 & 57 & 57 & 56 \\
\hline 1 & 6362 & 61 & 59 & 62 & 61 & 59 & 58 & 58 & 57 & 57 & 56 & 56 & 55 & 54 & 54 & 53 & 2 \\
\hline 2 & $60 \quad 58$ & 55 & 54 & 59 & 57 & 55 & 53 & 55 & 53 & 52 & 53 & 52 & 51 & 52 & 51 & 50 & 19 \\
\hline 3 & $57 \quad 54$ & 51 & 49 & 56 & 53 & 50 & 48 & 51 & 49 & 48 & 50 & 48 & 47 & 49 & 47 & 46 & 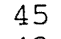 \\
\hline 4 & $54 \quad 50$ & 47 & 45 & 53 & 50 & 47 & 44 & 48 & 46 & 44 & 47 & 45 & 43 & 46 & 44 & 43 & 2 \\
\hline 5 & $52 \quad 47$ & 44 & 41 & 51 & 46 & 43 & 41 & 45 & 43 & 41 & 45 & 42 & 40 & 44 & 42 & 40 & 9 \\
\hline 6 & $49 \quad 44$ & 41 & 38 & 48 & 44 & 40 & 38 & 43 & 40 & 38 & 42 & 40 & 38 & 41 & 39 & 37 & \\
\hline 7 & $47 \quad 41$ & 38 & 36 & 46 & 41 & 38 & 36 & 40 & 37 & 35 & 40 & 37 & 35 & 39 & 37 & 35 & \\
\hline 8 & $44 \quad 39$ & 36 & 33 & 44 & 39 & 35 & 33 & 38 & 35 & 33 & 37 & 35 & 33 & 37 & 35 & 33 & \\
\hline 0 & $42 \quad 37$ & 33 & 31 & 41 & 36 & 33 & 31 & 36 & 33 & 31 & 35 & 33 & 31 & 35 & 33 & 31 & \\
\hline & $40 \quad 35$ & 31 & 29 & 40 & 34 & 31 & 29 & 34 & 31 & 29 & 34 & 31 & 29 & 33 & 31 & 29 & \\
\hline
\end{tabular}

ALL CANDELA, LUMENS, LUMINANCE, COEFFICIENT OF UTILIZATION AND VCP VALUES IN THIS REPORT ARE BASED ON RELATIVE PHOTOMETRY WHICH ASSUMES A BALLAST FACTOR OF 1.000. ANY CALCULATIONS PREPARED FROM THESE DATA SHOULD INCLUDE AN APPROPRIATE BALLAST FACTOR. 


\section{It boulder}

$\operatorname{FAX}(303) 449-5274$ INDEPENDENT TESTING LABORATORIES. INC. • 3386 LONGHORN ROAD, 8OULDER, COLORADO 80302 • PHONE (303) 442-1255 REPORT NUMBER: ITL40785 PREPARED FOR: U.S. ARMY CORPS OF ENGINEERS

DATE: $7-22-1993$

CATALOG NUMBER: DEEP CAN, BLACK BAFFLE

LUMINAIRE: FABRICATED CYLINDRICAL METAL HOUSING WITH BLACK PAINTED INTERIOR, BLACK PLASTIC LOWER BAFELE, WHITE PAINTED METAI TRIM RING.

LAMP: 15-WATT FLB15/TL COMPACT ELUORESCENT, RATED 70O LUMENS, VERTICAL BASE-UP POSITION.

MOUNTING : RECESSED

TOTAL INPUT WATTS $=16.1$ AT 120.0 VOLTS

\begin{tabular}{rcr} 
DEG & CANDELA & \multicolumn{2}{r}{ LUMENS } \\
0 & 64 & 6 \\
5 & 64 & 19 \\
15 & 68 & 30 \\
25 & 64 & 32 \\
35 & 51 & 26 \\
45 & 33 & 14 \\
55 & 15 & 3 \\
65 & 3 & 1 \\
75 & 1 & 0 \\
85 & 0 & \\
90 & 0 &
\end{tabular}

ZONAL LUMEN SUMMARY

$\begin{array}{lrrr}\text { ZONE } & \text { LUMENS } & \text { \&LAMP } & \text { \&FIXT } \\ 0-30 & 55 & 7.9 & 42.0 \\ 0-40 & 87 & 12.4 & 66.4 \\ 0-60 & 126 & 18.0 & 96.6 \\ 0-90 & 131 & 18.7 & 100.0 \\ 90-180 & 0 & 0.0 & 0.0 \\ 0-180 & 131 & 18.7 & 100.0\end{array}$

TOTAI LUMINAIRE EFFICIENCY = $18.7 \%$ CIE TYPE - DIRECT

LUMINAIRE SPACING CRITERION $=1.3$

LUMINOUS DIAMETER: 5.750

LUMINANCE DATA IN CANDELA/SQ M

ANGLE AVERAGE MAXIMUM MAX/AVG

IN DEG O-DEG O-DEG 0-DEG

45 2810. 16̈838. 6.0

55 1602. 13149. 8.2

$\begin{array}{lll}65 & 367 . & 3462 .\end{array}$

$75 \quad 184.256 .21 .4$

$85274.274 . \quad 1.0$
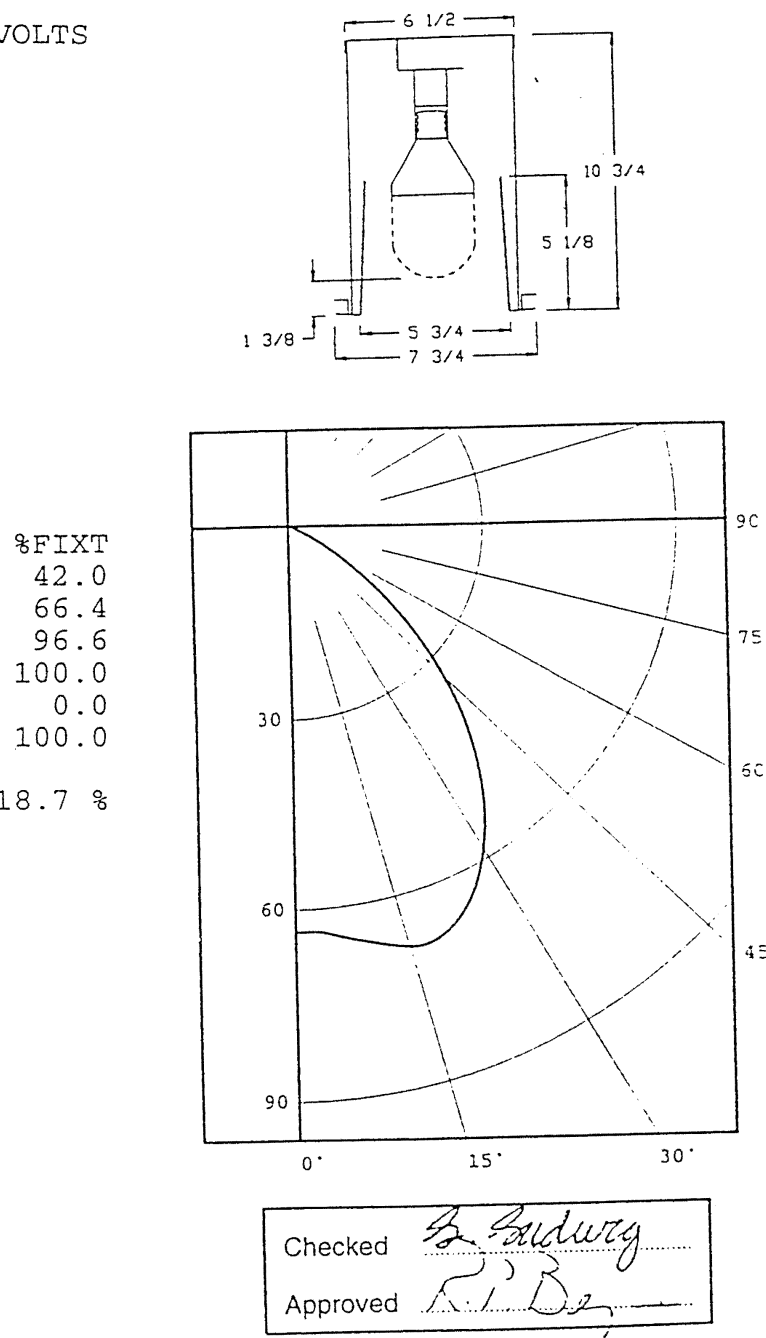

THIS REPORT IS BASED ON PUBLISHED INDUSTRY PROCEDURES. FIELD PERFORMANCE MAY DIFFER FROMLABORATORY PERFORMANC: 


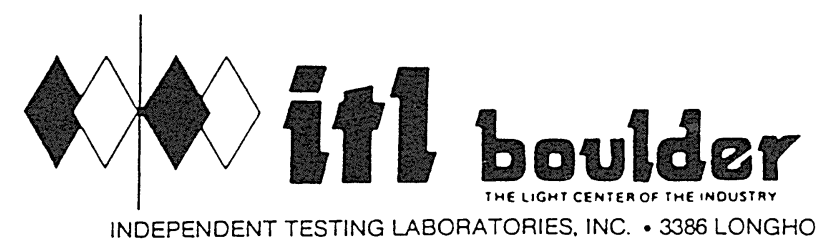

INDEPENDENT TESTING LABORATORIES, INC. • 3386 LONGHORN ROAD, BOULDER, COLORADO 80302 • PHONE (303) 442-125:

REPORT NUMBER: ITL40785

PREPARED FOR: U.S. ARMY CORPS OF ENGINEERS

DATE: $7-22-1993$

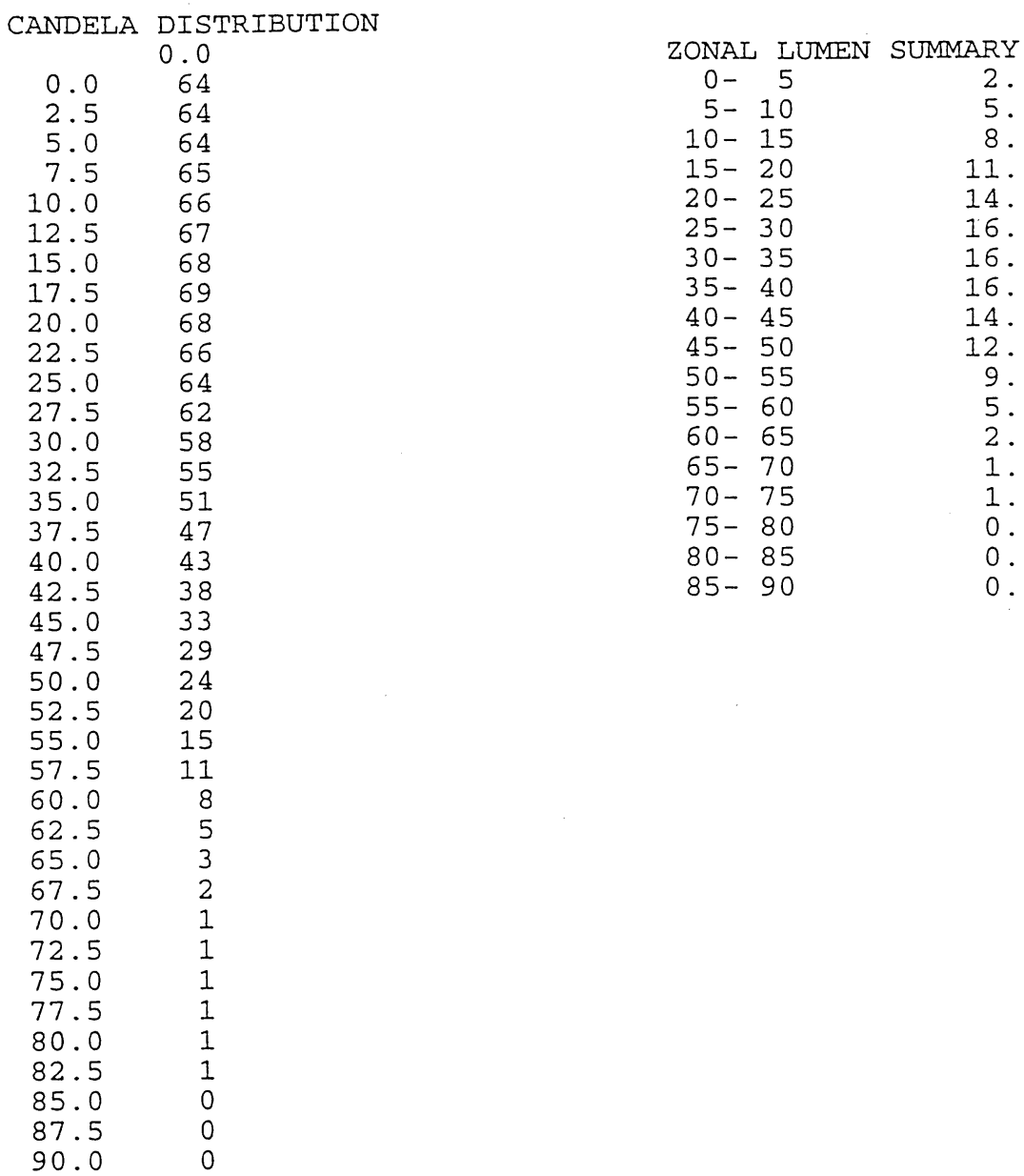




\section{- Itl boulder}

INDEPENDENT TESTING LABORATORIES, INC. • 3386 LONGHORN ROAD, BOULDER, COLORADO 80302 • PHONE (303) 442-12:
REPORT NUMBER: ITL40785

PREPARED FOR: U.S. ARMY CORPS OF ENGINEERS
DATE: $7-22-1993$

\author{
COEFFICIENTS OF UTILIZATION - ZONAL CAVITY. METHOD \\ EFEECTIVE FLOOR CAVITY REFLECTANCE 0.20
}

\begin{tabular}{rrrr}
\multicolumn{5}{c}{70} \\
70 & 50 & 30 & 10 \\
22 & 22 & 22 & 22 \\
20 & 20 & 19 & 19 \\
19 & 18 & 17 & 16 \\
18 & 16 & 15 & 14 \\
17 & 15 & 14 & 13 \\
15 & 14 & 12 & 11 \\
14 & 12 & 11 & 10 \\
13 & 11 & 10 & 9 \\
13 & 10 & 9 & 8 \\
12 & 10 & 8 & 7 \\
11 & 9 & 8 & 7
\end{tabular}

$\begin{array}{rrr} & 30 & \\ 50 & 30 & 10 \\ 20 & 20 & 20 \\ 18 & 18 & 18 \\ 17 & 16 & 16 \\ 15 & 15 & 14 \\ 14 & 13 & 12 \\ 13 & 12 & 11 \\ 12 & 11 & 10 \\ 11 & 10 & 9 \\ 10 & 9 & 8 \\ 9 & 8 & 7 \\ 9 & 8 & 7\end{array}$

$\begin{array}{rrrr} & 10 & & 0 \\ 50 & 30 & 10 & 0 \\ 19 & 19 & 19 & 19 \\ 18 & 17 & 17 & 17 \\ 16 & 16 & 15 & 15 \\ 15 & 14 & 14 & 13 \\ 14 & 13 & 12 & 12 \\ 12 & 12 & 11 & 11 \\ 11 & 11 & 10 & 9 \\ 11 & 10 & 9 & 9 \\ 10 & 9 & 8 & 8 \\ 9 & 8 & 7 & 7 \\ 8 & 7 & 7 & 6\end{array}$

ALI CANDELA, LUMENS, LUMINANCE, COEFFICIENT OF UTILIZATION AND VC? VALUES IN THIS REPORT ARE BASED ON RELATIVE PHOTOMETRY WHICH ASSUMES A BALLAST FACTOR OF 1.000. ANY CALCULATIONS PREPARED FROM THESE DATA SHOULD INCLUDE AN APPROPRIATE BALLAST FACTOR. 


\section{irl boulder}

INDEPENDENT TESTING LABORATORIES, INC. • 3386 LONGHORN ROAD, BOULDER, COLORADO 80302 • PHONE (303) 449-52i

REPORT NUMBER: ITL40786

PREPARED FOR: U.S. ARMY CORPS OF ENGINEERS

CATALOG NUMBER: DEEP CAN, BLACK BAFFLE

LUMINAIRE: FABRICATED CYLINDRICAL METAL HOUSING WITH BLACK PAINTED INTERIOR, BLACK PLASTIC LOWER BAFFLE, WHITE PAINTED METAL TRIM RING, OPEN BOTTOM.

LAMP: 11-WATT OSRAM DULUX EL COMPACT FLUORESCENT, RATED 600 LUMENS, VERTICAL BASE-UP POSITION.

MOUNTING : RECESSED

TOTAL INPUT WATTS $=11.6$ AT 120.0 VOLTS

\begin{tabular}{|c|c|c|c|}
\hline \multicolumn{4}{|c|}{ CANDELA DISTRIBUTION } \\
\hline & 0.0 & 22.5 & 45.0 \\
\hline 0 & 20 & 20 & 20 \\
\hline 5 & 21 & 21 & 22 \\
\hline 15 & 25 & 27 & 28 \\
\hline 25 & 30 & 34 & כ2 \\
\hline 35 & 22 & 27 & 29 \\
\hline 45 & 10 & 13 & 13 \\
\hline 55 & 1 & 1 & \\
\hline 65 & 1 & 1 & \\
\hline 75 & 0 & 0 & \\
\hline 85 & 0 & 0 & \\
\hline & 0 & 0 & \\
\hline
\end{tabular}

$\begin{array}{rr}67.5 & 90.0 \\ 20 & 20 \\ 22 & 22 \\ 28 & 27 \\ 34 & 32 \\ 28 & 25 \\ 14 & 13 \\ 2 & 2 \\ 1 & 1 \\ 0 & 0 \\ 0 & 0 \\ 0 & 0\end{array}$

ZONAL LUMEN SUMMARY ZONE LUMENS

$0-30$

$0-40$

25

$0-60$

$0-90$

$90-180$

$0-180$

\%LAMP
4.2
7.0
9.0
9.1
0.0
9.1

TOTAL LUMINAIRE EFFICIENCY = CIE TYPE - DIRECT
PLANE
: 0-DEG 90-DEG
SPACING CRITERIA :
1.51 .6
LUMINOUS DIAMETER:
5.750

LUMINANCE DATA IN CANDELA/SQ M ANGLE AVERAGE AVERAGE AVERAGE

IN DEG O-DEG 45-DEG 90-DEG

45 869. 1131. 1080.

$55 \quad 114 . \quad 166.2198$.

$\begin{array}{rrr}65 & 85 . & 85 .\end{array}$

7546.46.

8500.00.

ANGLE MAXIMIM MAXIMIM MAX/AVG MAX/AVG

IN DEG 0-DEG 90-DEG 0-DEG 90-DEG

$\begin{array}{rrrrr}45 & 15989 . & 21579 . & 18.4 & 20.0\end{array}$

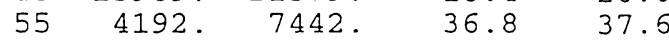

$\begin{array}{lllll}65 & 349 . & 427 . & 4.1 & 4.3\end{array}$

$75 \quad 78.7140 . \quad 1.7 \quad 3.0$

DATE : $7-26-1993$

FAX (303) 449-52i: 


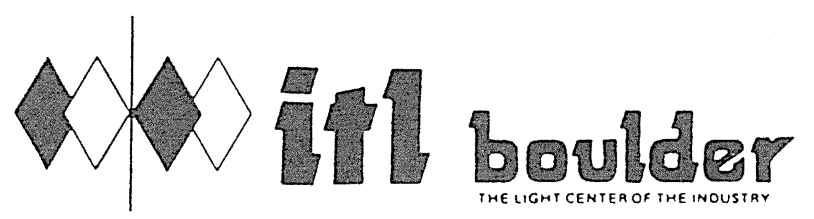

FAX (3C3) $44952:-$ INDEPENDENT TESTING LABORATORIES, INC. • 3386 LONGHORN ROAD. BOULDER, COLORADO 80302 • PHONE (303) 442-125:

REPORT NUMBER: ITL40786

PREPARED FOR: U.S. ARMY CORPS OF ENGINEERS

DATE: 7-26-1993

CANDELA DISTRIBUTION

$\begin{array}{rrrrrr}0.0 & 0.0 & 22.5 & 45.0 & 67.5 & 90.0 \\ 2.5 & 21 & 20 & 20 & 20 & 20 \\ 5.0 & 21 & 21 & 21 & 21 & 21 \\ 7.5 & 22 & 23 & 22 & 22 & 22 \\ 10.0 & 23 & 24 & 25 & 23 & 23 \\ 12.5 & 24 & 26 & 26 & 26 & 24 \\ 15.0 & 25 & 27 & 28 & 28 & 27 \\ 17.5 & 27 & 29 & 30 & 29 & 29 \\ 20.0 & 28 & 31 & 32 & 31 & 30 \\ 22.5 & 29 & 33 & 34 & 33 & 31 \\ 25.0 & 30 & 34 & 35 & 34 & 32 \\ 27.5 & 30 & 34 & 36 & 35 & 32 \\ 30.0 & 28 & 33 & 35 & 34 & 31 \\ 32.5 & 25 & 31 & 33 & 32 & 28 \\ 35.0 & 22 & 27 & 29 & 28 & 25 \\ 37.5 & 18 & 23 & 25 & 24 & 22 \\ 40.0 & 15 & 20 & 21 & 21 & 19 \\ 42.5 & 13 & 16 & 17 & 17 & 16 \\ 45.0 & 10 & 13 & 13 & 14 & 13 \\ 47.5 & 8 & 9 & 10 & 10 & 10 \\ 50.0 & 6 & 6 & 7 & 7 & 7 \\ 52.5 & 3 & 3 & 4 & 4 & 4 \\ 55.0 & 1 & 1 & 2 & 2 & 2 \\ 57.5 & 1 & 1 & 1 & 1 & 1 \\ 60.0 & 1 & 1 & 1 & 1 & 1 \\ 62.5 & 1 & 1 & 1 & 1 & 1 \\ 65.0 & 1 & 1 & 1 & 1 & 1 \\ 67.5 & 1 & 1 & 1 & 1 & 1 \\ 70.0 & 0 & 0 & 0 & 0 & 0 \\ 72.5 & 0 & 0 & 0 & 0 & 0 \\ 75.0 & 0 & 0 & 0 & 0 & 0 \\ 77.5 & 0 & 0 & 0 & 0 & 0 \\ 80.0 & 0 & 0 & 0 & 0 & 0 \\ 82.5 & 0 & 0 & 0 & 0 & 0 \\ 85.0 & 0 & 0 & 0 & 0 & 0 \\ 87.5 & 0 & 0 & 0 & 0 & 0 \\ 90.0 & 0 & 0 & 0 & 0 & 0\end{array}$

ZONAL LUMEN SUMMARY

$\begin{array}{ll}0-5 & 0 . \\ 5-10 & 2 .\end{array}$

$10-15 \quad 3$.

$15-20 \quad 5$.

$20-257$

$25-30 \quad 9$.

$30-359$.

$35-40 \quad 8$.

$40-456$

$45-50 \quad 4$

$50-55 \quad 2$.

$55-60 \quad 0$

$60-650$.

$65-70 \quad 0$.

$70-750$

$75-80 \quad 0$.

$\begin{array}{ll}80-85 & 0 . \\ 85-90 & 0 .\end{array}$ 


\section{- itl boulder}

INDEPENDENT TESTING LABORATORIES, INC. • 3386 LONGHORN ROAD. BOULDER, COLORADO 80302 • PHONE (303) 4:2-:-25:
DATE: 7-26-1993

PREPARED FOR: U.S. ARMY CORPS OF ENGINEERS

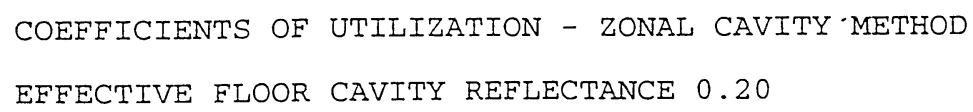

\begin{tabular}{rrrrr} 
RC & \multicolumn{5}{c}{80} \\
RW & 70 & 50 & 30 & 10 \\
& & & & \\
0 & 11 & 11 & 11 & 11 \\
1 & 10 & 10 & 10 & 9 \\
2 & 10 & 9 & 9 & 8 \\
3 & 9 & 8 & 8 & 7 \\
4 & 8 & 8 & 7 & 7 \\
5 & 8 & 7 & 6 & 6 \\
6 & 7 & 6 & 6 & 5 \\
7 & 7 & 6 & 5 & 5 \\
8 & 6 & 5 & 5 & 4 \\
9 & 6 & 5 & 4 & 4 \\
10 & 6 & 5 & 4 & 4
\end{tabular}

\begin{tabular}{rrrr}
\multicolumn{5}{c}{70} \\
70 & 50 & 30 & 10 \\
11 & 11 & 11 & 11 \\
10 & 10 & 10 & 9 \\
9 & 9 & 9 & 8 \\
9 & 8 & 8 & 7 \\
8 & 7 & 7 & 6 \\
8 & 7 & 6 & 6 \\
7 & 6 & 6 & 5 \\
7 & 6 & 5 & 5 \\
6 & 5 & 5 & 4 \\
6 & 5 & 4 & 4 \\
6 & 5 & 4 & 4
\end{tabular}

$\begin{array}{rrr} & 50 & \\ 50 & 30 & 10 \\ 10 & 10 & 10 \\ 9 & 9 & 9 \\ 9 & 8 & 8 \\ 8 & 8 & 7 \\ 7 & 7 & 6 \\ 7 & 6 & 6 \\ 6 & 6 & 5 \\ 6 & 5 & 5 \\ 5 & 5 & 4 \\ 5 & 4 & 4 \\ 4 & 4 & 4\end{array}$

$\begin{array}{rrr} & 30 & \\ 50 & 30 & 10 \\ 10 & 10 & 10 \\ 9 & 9 & 9 \\ 8 & 8 & 8 \\ 8 & 7 & 7 \\ 7 & 7 & 6 \\ 7 & 6 & 6 \\ 6 & 6 & 5 \\ 6 & 5 & 5 \\ 5 & 5 & 4 \\ 5 & 4 & 4 \\ 4 & 4 & 4\end{array}$

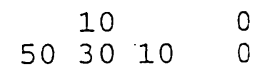

$\begin{array}{llll}9 & 9 & 9 & 9\end{array}$

$\begin{array}{llll}9 & 9 & 9 & 8\end{array}$

$\begin{array}{llll}8 & 8 & 8 & 8\end{array}$

$\begin{array}{llll}7 & 7 & 7 & 7\end{array}$

$\begin{array}{llll}7 & 7 & 6 & 6\end{array}$

$\begin{array}{lll}6 & 6 & 5\end{array}$

6555

$\begin{array}{llll}5 & 5 & 5 & 4\end{array}$

$\begin{array}{llll}5 & 5 & 4 & 4\end{array}$

$\begin{array}{llll}5 & 4 & 4 & 4\end{array}$

ALL CANDELA, LUMENS, LUMINANCE, COEFFICIENT OF UTILIZATION AND VCD VALUES IN THIS REPORT ARE BASED ON RELATIVE PHOTOMETRY WHICH ASSUMES A BALLAST FACTOR OF 1.000. ANY CALCULATIONS PREPARED FROM THESE DATA SHOULD INCLUDE AN APPROPRIATE BALLAST FACTOR. 


\section{All boulder}

FAX (303) 449-52:INDEPENDENT TESTING LABORATORIES, INC. • 3386 LONGHORN ROAD, BOULDER, COLORADO 80302 • PHONE (303) 442-125: REPORT NUMBER: ITL40787

PREPARED FOR: U.S. ARMY CORPS OF ENGINEERS

CATALOG NUMBER: DEEP CAN, BLACK BAFFLE

LUMINAIRE: FABRICATED CYLINDRICAL METAL HOUSING WITH BLACK PAINTED INTERIOR, BLACK PLASTIC LOWER BAFFLE, WHITE PAINTED METAL TRIM RING, OPEN BOTTOM.

LAMP: 13-WATT DOUBLE TWIN TUBE COMPACT FLUORESCENT WITH MODULAR BALLAST/ SOCKET ADAPTER, RATED 860 LUMENS, VERTICAL BASE-UP POSITION.

BALLAST: SYLVANIA MODULAR BALLAST/SOCKET ADAPTER, 535F LAMP HOLDER FITTIN MODEL AE013

MOUNTING: RECESSED

TOTAL INPUT WATTS $=17.2$ AT 120.0 VOLTS

CANDELA DISTRIBUTION

$\begin{array}{rrrrrr} & 0.0 & 22.5 & 45.0 & 67.5 & 90.0 \\ 0 & 20 & 20 & 20 & 20 & 20 \\ 5 & 21 & 21 & 22 & 22 & 22 \\ 15 & 29 & 31 & 32 & 30 & 29 \\ 25 & 38 & 42 & 43 & 41 & 38 \\ 35 & 37 & 44 & 46 & 44 & 39 \\ 45 & 25 & 31 & 34 & 33 & 28 \\ 55 & 13 & 16 & 17 & 17 & 16 \\ 65 & 2 & 2 & 3 & 3 & 3 \\ 75 & 1 & 1 & 0 & 1 & 1 \\ 85 & 0 & 0 & 0 & 0 & 0 \\ 90 & 0 & 0 & 0 & 0 & 0\end{array}$

ZONAI LUMEN SUMMARY

$\begin{array}{rr}\text { ZONE } & \text { LUMENS } \\ 0-30 & 30 \\ 0-40 & 57 \\ 0-60 & 95 \\ 0-90 & 98 \\ 90-180 & 0 \\ 0-180 & 98\end{array}$

8LAMP
3.5
6.6
11.0
11.4
0.0
11.4

FLUX

TOTAI LUMINAIRE EFFICIENCY = CIE TYPE - DIRECT

$\begin{array}{llrr}\text { PLANE } & \text { : } & \text { DEG } & 90-D E G \\ \text { SPACING CRITERIA : } & 1.9 & 2.0 \\ \text { LUMINOUS DIAMETER: } & 5.750 & \end{array}$

LUMINANCE DATA IN CANDELA/SQ M ANGLE AVERAGE AVERAGE AVERAGE

IN DEG O-DEG 45-DEG '90-DEG

45 2143. 2852. 2396.

55 1311. 1779.1623.

$65 \quad 339 . \quad 353 . \quad 424$.

$\begin{array}{rrrr}75 & 115 . & 92 . & 115 .\end{array}$

ANGLE MAXIMUM MAXIMUM MAX/AVG MAX/AVG

IN DEG $0-D E G$ 90-DEG $0-D E G$ 90-DEG

$\begin{array}{rrrrr}45 & 23347 . & 27456 . & 10.9 & 11.5 \\ 55 & 13560 . & 20358 . & 10.4 & 12.5 \\ 65 & 1177 . & 4307 . & 3.5 & 10.2 \\ 75 & 219 . & 362 . & 1.9 & 3.1 \\ 85 & 31 . & 65 . & 0.0 & 0.0\end{array}$

DATE : $7-26-1993$

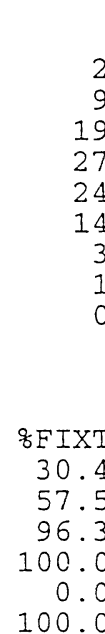

11.48
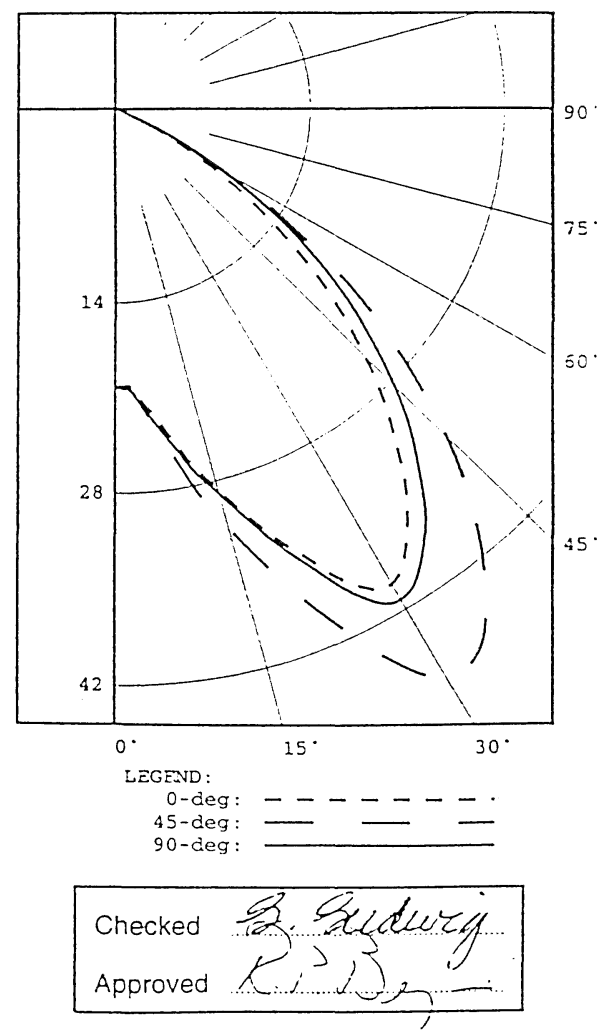

THIS REPORT IS BASED ON PUBLISHED INOUSTRY PROCEDURES. FIELD PERFORMANCE MAY DIFFER FROM LABORATORY PERFORMANCE 


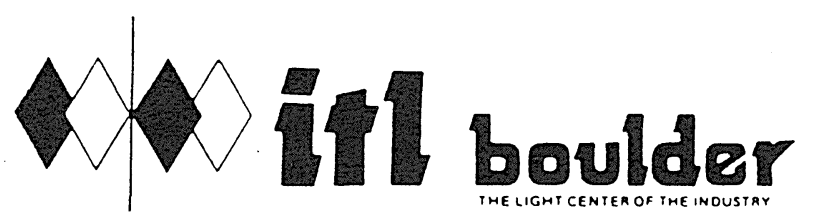

$\operatorname{FAX}(303) 449-527:$ INDEPENDENT TESTING LABORATORIES, INC. • 3386 LONGHORN ROAD, BOULDER. COLORADO 80302 • PHONE (303) $442-1255$

REPORT NUMBER: ITL40787

PREPARED FOR: U.S. ARMY CORPS OF ENGINEERS

DATE : $7-26-1993$

CANDELA DISTRIBUTION

$\begin{array}{rrrrrr}0.0 & 0.0 & 22.5 & 45.0 & 67.5 & 90.0 \\ 2.5 & 20 & 20 & 20 & 20 & 20 \\ 5.0 & 21 & 21 & 20 & 20 & 20 \\ 7.5 & 23 & 23 & 24 & 22 & 22 \\ 10.0 & 25 & 26 & 26 & 26 & 23 \\ 12.5 & 27 & 28 & 29 & 28 & 27 \\ 15.0 & 29 & 31 & 32 & 30 & 29 \\ 17.5 & 31 & 33 & 34 & 33 & 31 \\ 20.0 & 33 & 36 & 37 & 36 & 34 \\ 22.5 & 35 & 39 & 40 & 38 & 36 \\ 25.0 & 38 & 42 & 43 & 41 & 38 \\ 27.5 & 39 & 44 & 46 & 44 & 40 \\ 30.0 & 40 & 45 & 48 & 46 & 41 \\ 32.5 & 39 & 45 & 48 & 46 & 41 \\ 35.0 & 37 & 44 & 46 & 44 & 39 \\ 37.5 & 34 & 41 & 44 & 42 & 37 \\ 40.0 & 31 & 38 & 41 & 39 & 34 \\ 42.5 & 29 & 35 & 37 & 36 & 31 \\ 45.0 & 25 & 31 & 34 & 33 & 28 \\ 47.5 & 22 & 28 & 30 & 29 & 25 \\ 50.0 & 19 & 24 & 26 & 25 & 22 \\ 52.5 & 16 & 20 & 22 & 21 & 19 \\ 55.0 & 13 & 16 & 17 & 17 & 16 \\ 57.5 & 10 & 12 & 13 & 13 & 12 \\ 60.0 & 7 & 8 & 9 & 9 & 9 \\ 62.5 & 4 & 5 & 6 & 6 & 6 \\ 65.0 & 2 & 2 & 3 & 3 & 3 \\ 67.5 & 1 & 1 & 1 & 1 & 1 \\ 70.0 & 1 & 1 & 1 & 1 & 1 \\ 72.5 & 1 & 1 & 1 & 1 & 1 \\ 75.0 & 1 & 1 & 0 & 1 & 1 \\ 77.5 & 0 & 0 & 0 & 0 & 0 \\ 80.0 & 0 & 0 & 0 & 0 & 0 \\ 82.5 & 0 & 0 & 0 & 0 & 0 \\ 85.0 & 0 & 0 & 0 & 0 & 0 \\ 87.5 & 0 & 0 & 0 & 0 & 0 \\ 90.0 & 0 & 0 & 0 & 0 & 0\end{array}$

ZONAL LUMEN SUMMARY

$\begin{array}{ll}0-5 & 0 \\ 5-10 & 2\end{array}$

$10-15 \quad 3$.

$15-20 \quad 5$.

$20-258$.

$25-30 \quad 11$.

$30-35 \quad 13$.

$35-40 \quad 14$.

$40-45 \quad 13$.

$45-50 \quad 11$.

$50-559$.

$55-60 \quad 6$.

$60-653$.

$65-70 \quad 1$

$70-750$.

$75-80 \quad 0$.

$80-85 \quad 0$. 


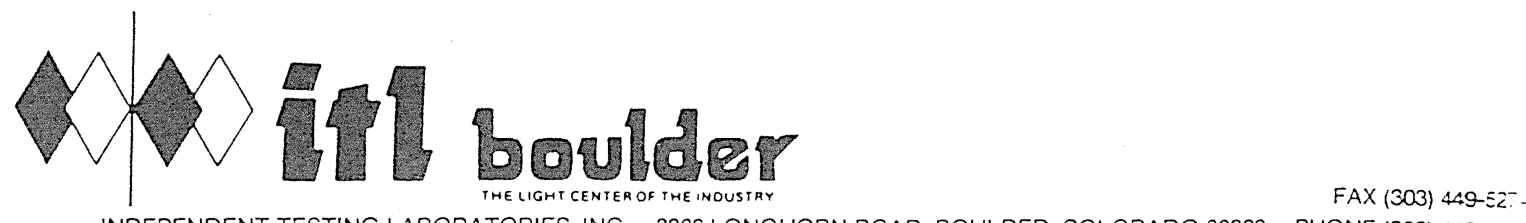
INDEPENDENT TESTING LABORATORIES, INC. • 3386 LONGHORN ROAD, BOULDER, COLORADO 80302 • PHONE (303) 442-:2:

REPORT NUMBER: ITL40787

DATE : 7-26-1993

PREPARED FOR: U.S. ARMY CORPS OF ENGINEERS

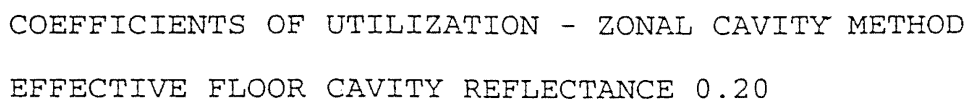

\begin{tabular}{|c|c|c|c|c|c|c|c|c|c|c|c|c|c|c|c|c|c|}
\hline$R C$ & \multicolumn{3}{|c|}{80} & \multicolumn{4}{|c|}{70} & \multicolumn{3}{|c|}{50} & \multicolumn{3}{|c|}{30} & \multicolumn{3}{|c|}{10} & 0 \\
\hline 70 & 50 & 30 & 10 & 70 & 50 & 30 & 10 & 50 & 30 & 10 & 50 & 30 & 10 & 50 & 30 & 10 & 0 \\
\hline 14 & 14 & 14 & 14 & 13 & 13 & 13 & 13 & 13 & 13 & 13 & 12 & 12 & 12 & 12 & 12 & 12 & 12 \\
\hline 13 & 12 & 12 & 12 & 13 & 12 & 12 & 11 & 12 & 11 & 11 & 11 & 11 & 11 & 11 & 11 & 10 & 10 \\
\hline 12 & 11 & 10 & 10 & 12 & 11 & 10 & 10 & 10 & 10 & 10 & 10 & 10 & 9 & 10 & 9 & 9 & 9 \\
\hline 11 & 10 & 9 & 8 & 11 & 10 & 9 & 8 & 9 & 9 & 8 & 9 & 9 & 8 & 9 & 8 & 8 & 8 \\
\hline 10 & 9 & 8 & 7 & 10 & 9 & 8 & 7 & 8 & 8 & 7 & 8 & 8 & 7 & 8 & 7 & 7 & 7 \\
\hline 9 & 8 & 7 & 6 & 9 & 8 & 7 & 6 & 8 & 7 & 6 & 7 & 7 & 6 & 7 & 7 & 6 & 6 \\
\hline 9 & 7 & 6 & 6 & 8 & 7 & 6 & 6 & 7 & 6 & 5 & 7 & 6 & 5 & 7 & 6 & 5 & 5 \\
\hline 8 & 6 & 6 & 5 & 8 & 6 & 6 & 5 & 6 & 5 & 5 & 6 & 5 & 5 & 6 & 5 & 5 & \\
\hline 7 & 6 & 5 & 4 & 7 & 6 & 5 & 4 & 6 & 5 & 4 & 6 & 5 & 4 & 5 & 5 & 4 & \\
\hline 7 & 5 & 4 & 4 & 7 & 5 & 4 & 4 & 5 & 4 & 4 & 5 & 4 & 4 & 5 & 4 & 4 & \\
\hline 6 & 5 & 4 & 3 & 6 & 5 & 4 & 3 & 5 & 4 & 3 & 5 & 4 & 3 & 5 & 4 & 3 & \\
\hline
\end{tabular}

ALL CANDELA, LUMENS, LUMINANCE, COEFFICIENT OF UTILIZATION AND VCD VALUES IN THIS REPORT ARE BASED ON RELATIVE PHOTOMETRY WHICH ASSUMES A BALLAST FACTOR OF 1.000. ANY CALCULATIONS PREPARED FROM THESE DATA SHOULD INCLUDE AN APPROPRIATE BALLAST FACTOR. 


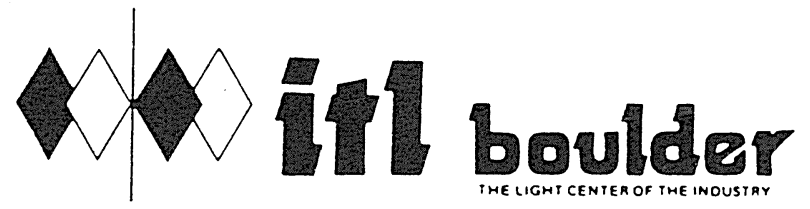

$\operatorname{FAX}(303) 449-5274$ INDEPENDENT TESTING LABORATORIES, INC. • 3386 LONGHORN ROAD, BOULDER, COLORADO 80302 • PHONE (303) 442-1255 REPORT NUMBER: ITL40788

PREPARED FOR: U.S. ARMY CORPS OF ENGINEERS

DATE: $7-27-1993$

CATALOG NUMBER: DEEP CAN, BLACK BAFFLE

LUMINAIRE: FABRICATED CYLINDRICAL METAL HOUSING WITH BLACK PAINTED INTERIOR, BLACK PLASTIC LOWER BAFFLE, WHITE PAINTED METAL TRIM RING, OPEN BOTTOM.

LAMP: 13-WATT LUMATECH RAS-130 REFLECT-A-STAR WITH 13-WATT QUAD TUBE FLUORESCENT, RATED 860 LUMENS, VERTICAL BASE-UP POSITION. MOUNTING : RECESSED TOTAL INPUT WATTS $=15.5$ AT 120.0 VOLTS

$\begin{array}{rrr}\text { DEG } & \text { CANDELA } & \text { LUMENS } \\ 0 & 228 & \\ 5 & 225 & 21 \\ 15 & 192 & 54 \\ 25 & 133 & 61 \\ 35 & 83 & 51 \\ 45 & 43 & 33 \\ 55 & 16 & 15 \\ 65 & 3 & 3 \\ 75 & 0 & 1 \\ 85 & 0 & 0 \\ 90 & 0 & \end{array}$

ZONAI LUMEN SUMMARY

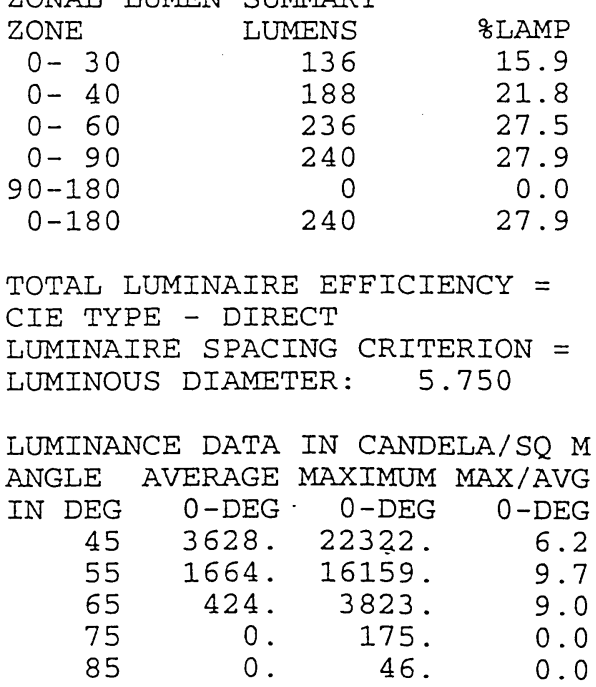

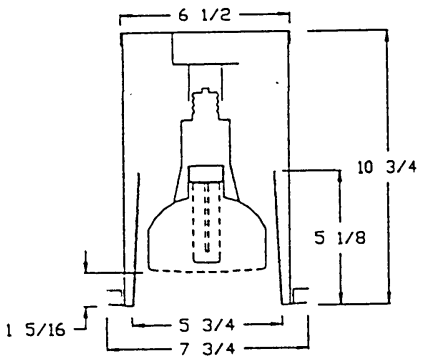

54

61

33

3

1

$\begin{array}{rr} & \\ \text { 8LAMP } & \text { \%FIXT } \\ 15.9 & 56.9 \\ 21.8 & 78.3 \\ 27.5 & 98.6 \\ 27.9 & 100.0 \\ 0.0 & 0.0 \\ 27.9 & 100.0\end{array}$

27.98

0.8

\section{8}

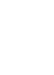




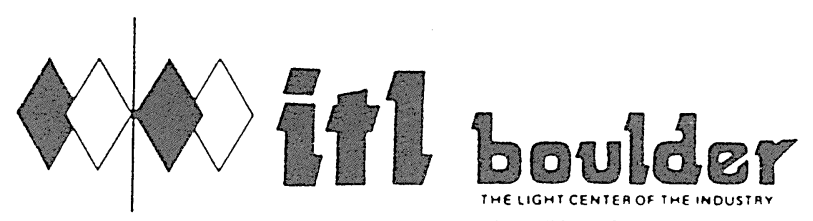

INDEPENDENT TESTING LABORATORIES, INC • 3386 LONGHORN ROAD. B

FAX (303) 449-5274 INDEPENDENT TESTING LABORATORIES. INC. • 3386 LONGHORN ROAD. BOULDER. COLORADO 80302 - PHONE (303) 442-1255

REPORT NUMBER: ITL 40788

PREPARED FOR: U.S. ARMY CORPS OF ENGINEERS

DATE: $7-27-1993$

\begin{tabular}{|c|c|c|c|c|}
\hline CANDELA & $\begin{array}{l}\text { DISTRIBUTION } \\
0.0\end{array}$ & ZONAI & LUMEN & SUMMARY \\
\hline 0.0 & 228 & $0-$ & 5 & 5. \\
\hline 2.5 & 227 & $5-$ & 10 & 16. \\
\hline 5.0 & 225 & $10-$ & 15 & 24 . \\
\hline 7.5 & 222 & $15-?$ & 20 & 29. \\
\hline 10.0 & 215 & $20-$ & 25 & 31. \\
\hline 12.5 & 205 & $25-$ & 30 & 30. \\
\hline 15.0 & 192 & $30-$ & 35 & 28. \\
\hline 17.5 & 178 & $35-$ & 40 & 24. \\
\hline 20.0 & 163 & $40-$ & 45 & 19. \\
\hline 22.5 & 148 & $45-$ & 50 & 14. \\
\hline 25.0 & 133 & $50-$ & 55 & 10. \\
\hline 27.5 & 120 & $55-$ & 60 & 6. \\
\hline 30.0 & 106 & $60-$ & 65 & 2 \\
\hline 32.5 & 94 & $65-$ & 70 & 1 \\
\hline 35.0 & 83 & $70-$ & 75 & 1 \\
\hline 37.5 & 71 & $75-$ & 80 & 0 . \\
\hline 40.0 & 61 & $80-$ & 85 & 0 . \\
\hline 42.5 & 52 & $85-$ & 90 & 0 . \\
\hline 45.0 & 43 & & & \\
\hline 47.5 & 35 & & & \\
\hline 50.0 & 28 & & & \\
\hline 52.5 & 22 & & & \\
\hline 55.0 & 16 & & & \\
\hline 57.5 & 12 & & & \\
\hline 60.0 & 8 & & & \\
\hline 62.5 & 5 & & & \\
\hline 65.0 & 3 & & & \\
\hline 67.5 & 1 & & & \\
\hline 70.0 & 1 & & & \\
\hline 72.5 & 1 & & & \\
\hline 75.0 & 0 & & & \\
\hline 77.5 & 0 & & & \\
\hline 80.0 & 0 & & & \\
\hline 82.5 & 0 & & & \\
\hline 85.0 & 0 & & & \\
\hline 87.5 & 0 & & & \\
\hline 90.0 & 0 & & & \\
\hline
\end{tabular}




\section{itl boulder}

INDEPENDENT TESTING LABORATORIES, INC. • 3386 LONGHORN ROAD, BOULDER, COLORADO 80302 • PHONE (303) 442-125
DATE: $7-27-1993$

PREPARED FOR: U.S. ARMY CORPS OF ENGINEERS

COEFFICIENTS OF UTILIZATION - ZONAL CAVITY METHOD

EFFECTIVE FLOOR CAVITY REFLECTANCE 0.20

\begin{tabular}{|c|c|c|c|c|c|c|c|c|c|c|c|c|c|c|c|c|c|c|}
\hline $\mathrm{RC}$ & \multicolumn{4}{|c|}{80} & \multicolumn{4}{|c|}{70} & \multicolumn{3}{|c|}{50} & \multicolumn{3}{|c|}{30} & \multicolumn{3}{|c|}{10} & 0 \\
\hline RW & 70 & 50 & 30 & 10 & 70 & 50 & 30 & 10 & 50 & 30 & 10 & 50 & 30 & 10 & 50 & 30 & 10 & 0 \\
\hline 0 & 33 & 33 & 33 & 33 & 32 & 32 & 32 & 32 & 31 & 31 & 31 & 30 & 30 & 30 & 28 & 28 & 28 & 28 \\
\hline 1 & 31 & 30 & 30 & 29 & 31 & 30 & 29 & 29 & 29 & 28 & 28 & 28 & 27 & 27 & 27 & 26 & 26 & 26 \\
\hline 2 & 30 & 28 & 27 & 26 & 29 & 28 & 26 & 25 & 27 & 26 & 25 & 26 & 25 & 24 & 25 & 24 & 24 & 23 \\
\hline 3 & 28 & 26 & 24 & 23 & 27 & 25 & 24 & 23 & 25 & 23 & 22 & 24 & 23 & 22 & 23 & 22 & 22 & 21 \\
\hline 4 & 26 & 24 & 22 & 21 & 26 & 23 & 22 & 21 & 23 & 21 & 20 & 22 & 21 & 20 & 22 & 21 & 20 & 19 \\
\hline 5 & 25 & 22 & 20 & 19 & 24 & 22 & 20 & 19 & 21 & 20 & 19 & 21 & 19 & 18 & 20 & 19 & 18 & 18 \\
\hline 6 & 23 & 20 & 19 & 17 & 23 & 20 & 18 & 17 & 20 & 18 & 17 & 19 & 18 & 17 & 19 & 18 & 17 & 16 \\
\hline 7 & 22 & 19 & 17 & 16 & 21 & 19 & 17 & 16 & 18 & 17 & 16 & 18 & 17 & 16 & 18 & 16 & 15 & 5 \\
\hline 8 & 21 & 18 & 16 & 15 & 20 & 18 & 16 & 15 & 17 & 16 & 14 & 17 & 15 & 14 & 17 & 15 & 14 & \\
\hline 9 & 20 & 17 & 15 & 14 & 19 & 16 & 15 & 14 & 16 & 15 & 13 & 16 & 14 & 13 & 16 & 14 & 13 & 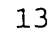 \\
\hline 10 & 19 & 16 & 14 & 13 & 18 & 15 & 14 & 13 & 15 & 14 & 13 & 15 & 14 & 13 & 15 & 13 & 13 & 2 \\
\hline
\end{tabular}

ALI CANDELA, LUMENS, LUMINANCE, COEFFICIENT OF UTILIZATION AND VCP VALUES IN THIS REPORT ARE BASED ON RELATIVE PHOTOMETRY WHICH ASSUMES A BALLAST FACTOR OF 1.000. ANY CALCULATIONS PREPARED FROM THESE DATA SHOULD INCLUDE AN APPROPRIATE BALLAST FACTOR. 


\section{All boulder}

FAX (303) $449527:$ INDEPENDENT TESTING LABORATORIES. INC. • 3386 LONGHORN ROAD, BOULDER, COLORADO 80302 • PHONE (303) 442-1255 REPORT NUMBER: ITL40789

PREPARED FOR: U.S. ARMY CORPS OF ENGINEERS

CATALOG NUMBER: DEEP CAN, BLACK BAFELE

LUMINAIRE: FABRICATED CYLINDRICAI METAL HOUSING WITH BLACK PAINTED INTERIOR, BLACK PLASTIC LOWER BAFFLE, WHITE PAINTED METAI TRIS RING, OPEN BOTTOM.

LAMP: 15-WATT DULUX EL REFLECTOR COMPACT FLUORESCENT, RATED 900 LUMENS, VERTICAL BASE-UP POSITION

MOUNTING: RECESSED

TOTAI INPUT WATTS $=15.1$ AT 120.0 VOLTS

\begin{tabular}{rrrrrr}
\multicolumn{6}{c}{ CANDELA } \\
\multicolumn{7}{c}{ DISTRIBUTION } \\
0 & 505 & 22.5 & 45.0 & 67.5 & 90.0 \\
5 & 500 & 502 & 504 & 506 & 506 \\
15 & 458 & 457 & 452 & 455 & 458 \\
25 & 366 & 360 & 351 & 358 & 364 \\
35 & 252 & 242 & 230 & 241 & 251 \\
45 & 140 & 134 & 123 & 134 & 142 \\
55 & 56 & 54 & 49 & 55 & 58 \\
65 & 5 & 5 & 5 & 5 & 5 \\
75 & 1 & 1 & 1 & 1 & 1 \\
85 & 0 & 0 & 0 & 0 & 0 \\
90 & 0 & 0 & 0 & 0 & 0
\end{tabular}

ZONAL LUMEN SUMMARY

$\begin{array}{crr}\text { ZONE } & \text { LUMENS } & \text { \% LAMP } \\ 0-30 & 340 & 37 \cdot 8 \\ 0-40 & 490 & 54 \cdot 5 \\ 0-60 & 642 & 71 \cdot 3 \\ 0-90 & 649 & 72 \cdot 1 \\ 90-180 & 0 & 0.0 \\ 0-180 & 649 & 72 \cdot 1\end{array}$

TOTAL LUMINAIRE EFEICIENCY = CIE TYPE - DIRECT

$\begin{array}{lrrr}\text { PLANE } & : & 0-D E G & 90-D E G \\ \text { SPACING CRITERIA } & : & 1.0 & 1.0\end{array}$

LUMINOUS DIAMETER: 5.750

LUMINANCE DATA IN CANDELA/SQ M

ANGLE AVERAGE AVERAGE AVERAGE

IN DEG 0-DEG 45-DEG 90-DEG

45 11812. 10378. . 11981.

55 5825. 5097. 6033.

65706.706 .706$.

$75 \quad 231.231 .231$.

850.0 . 0.

ANGLE MAXIMUM MAXIMUM MAX/AVG MAX/AVG

IN DEG $0-D E G$ 90-DEG 0 -DEG 90-DEG

$\begin{array}{lllll}45 & 52224.52349 . & 4.4 & 4.4\end{array}$

$\begin{array}{lllll}55 & 39522 . & 43062 . & 6.8 & 7.1\end{array}$

$\begin{array}{lllll}65 & 4181 . & 12369 . & 5.9 & 17.5\end{array}$

$\begin{array}{lllll}75 & 307 . & 419 . & 1.3 & 1.8\end{array}$

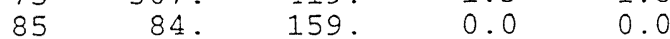

ELUX

48
128

164

151

103

48

6

0

\&FIXT

52.3

75.5

98.8

100.0

0.0

100.0

$72.1 \%$

DATE: 7-29-1993 


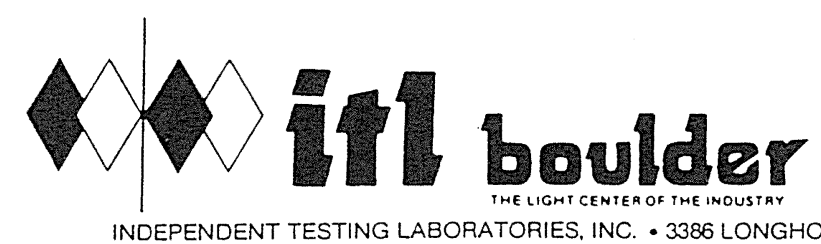

INDEPENDENT TESTING LABORATORIES, INC. • 3386 LONGHORN ROAD, BOULDER, COLORADO 80302 • PHONE (303) 442-1

REPORT NUMBER: ITL40789

PREPARED FOR: U.S. ARMY CORPS OF ENGINEERS

DATE: 7-29-1993

CANDELA DISTRIBUTION

\begin{tabular}{|c|c|c|c|c|c|c|c|}
\hline 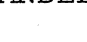 & 0.0 & 22.5 & 45.0 & 67.5 & 90.0 & ZONAI LUMEN & SUMMARY \\
\hline 0.0 & 505 & 505 & 505 & 505 & 505 & $0-5$ & 12 \\
\hline 2.5 & 503 & 504 & 506 & 506 & 506 & $5-10$ & 36. \\
\hline 5.0 & 500 & 502 & 504 & 506 & 506 & $10-15$ & 56. \\
\hline 7.5 & 497 & 498 & 499 & 500 & 500 & $15-20$ & 71. \\
\hline 10.0 & 488 & 490 & 488 & 488 & 488 & $20-25$ & 80. \\
\hline 12.5 & 476 & 476 & 472 & 473 & 474 & $25-30$ & 84. \\
\hline 15.0 & 458 & 457 & 452 & 455 & 458 & $30-35$ & 80. \\
\hline 17.5 & 438 & 434 & 428 & 433 & 438 & $35-40$ & 71. \\
\hline 20.0 & 414 & 409 & 401 & 407 & 413 & $40-45$ & 58. \\
\hline 22.5 & 391 & 385 & 376 & 383 & 389 & $45-50$ & 45. \\
\hline 25.0 & 366 & 360 & 351 & 358 & 364 & $50-55$ & 31. \\
\hline 27.5 & 338 & 333 & 324 & 332 & 337 & $55-60$ & 17. \\
\hline 30.0 & 308 & 303 & 294 & 303 & 308 & $60-65$ & 5 . \\
\hline 32.5 & 280 & 273 & 262 & 272 & 279 & $65-70$ & 1 . \\
\hline 35.0 & 252 & 242 & 230 & 241 & 251 & $70-75$ & 1. \\
\hline 37.5 & 223 & 212 & 199 & 212 & 223 & $75-80$ & 0 . \\
\hline 40.0 & 194 & 184 & 170 & 184 & 195 & $80-85$ & 0 . \\
\hline 42.5 & 166 & 158 & 145 & 158 & 167 & $85-90$ & 0 . \\
\hline 45.0 & 140 & 134 & 123 & 134 & 142 & & \\
\hline 47.5 & 117 & 112 & 102 & 113 & 119 & & \\
\hline 50.0 & 95 & 92 & 84 & 92 & 97 & & \\
\hline 52.5 & 75 & 72 & 66 & 73 & 77 & & \\
\hline 55.0 & 56 & 54 & 49 & 55 & 58 & & \\
\hline 57.5 & 38 & 37 & 34 & 38 & 41 & & \\
\hline 60.0 & 23 & 22 & 21 & 23 & 24 & & \\
\hline 62.5 & 12 & 11 & 11 & 11 & 12 & & \\
\hline 65.0 & 5 & 5 & 5 & 5 & 5 & & \\
\hline 67.5 & 2 & 2 & 2 & 2 & 2 & & \\
\hline 70.0 & 1 & 1 & 1 & 1 & 1 & & \\
\hline 72.5 & 1 & 1 & 1 & 1 & 1 & & \\
\hline 75.0 & 1 & 1 & 1 & 1 & 1 & & \\
\hline 77.5 & 0 & 1 & 1 & 1 & 1 & & \\
\hline 80.0 & 0 & 0 & 0 & 0 & 0 & & \\
\hline 82.5 & 0 & 0 & 0 & 0 & 0 & & \\
\hline 85.0 & 0 & 0 & 0 & 0 & 0 & & \\
\hline 87.5 & 0 & 0 & 0 & 0 & 0 & & \\
\hline 90.0 & 0 & 0 & 0 & 0 & 0 & & \\
\hline
\end{tabular}




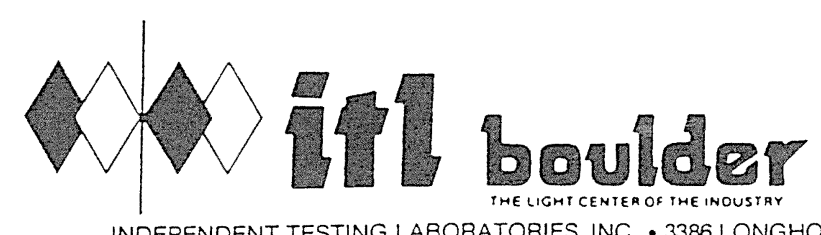

INDEPENDENT TESTING LABORATORIES, INC. • 3386 LONGHORN ROAD, BOULDER, COLORADO 80302 • PHONE (303) 442-12

FAX (303) 449-52-
HONE (303) $442-12=$

REPORT NUMBER: ITL40789

DATE: $7-29-1993$

PREPARED FOR: U.S. ARMY CORPS OF ENGINEERS

COEFFICIENTS OF UTILIZATION - ZONAL CAVITY·METHOD

EFFECTIVE FLOOR CAVITY REFLECTANCE 0.20

\begin{tabular}{|c|c|c|c|c|c|c|c|c|c|c|c|c|c|c|c|c|c|}
\hline $\mathrm{RC}$ & \multicolumn{3}{|c|}{80} & \multicolumn{4}{|c|}{70} & \multicolumn{3}{|c|}{50} & \multicolumn{3}{|c|}{30} & \multicolumn{3}{|c|}{10} & 0 \\
\hline RW & $70 \quad 50$ & 30 & 10 & 70 & 50 & 30 & 10 & 50 & 30 & 10 & 50 & 30 & 10 & 50 & 30 & 1.0 & 0 \\
\hline 0 & 8686 & 86 & 86 & 84 & 84 & 84 & 84 & 80 & 80 & 80 & 77 & 77 & 77 & 74 & 74 & 74 & 72 \\
\hline 1 & 8179 & 77 & 75 & 79 & 77 & 76 & 74 & 74 & 73 & 72 & 72 & 71 & 69 & 69 & 68 & 67 & 6 \\
\hline 2 & $76 \quad 72$ & 69 & 66 & 75 & 71 & 68 & 65 & 69 & 66 & 64 & 66 & 64 & 62 & 64 & 63 & 61 & 0 \\
\hline 3 & $72 \quad 66$ & 62 & 59 & 70 & 65 & 61 & 58 & 63 & 60 & 57 & 61 & 59 & 56 & 60 & 57 & 55 & 4 \\
\hline 4 & $67 \quad 61$ & 56 & 53 & 66 & 60 & 56 & 52 & 58 & 55 & 52 & 57 & 54 & 51 & 55 & 53 & 50 & 9 \\
\hline 5 & 6356 & 51 & 47 & 62 & 55 & 51 & 47 & 54 & 50 & 47 & 53 & 49 & 46 & 51 & 48 & 46 & 5 \\
\hline 6 & $59 \quad 52$ & 47 & 43 & 58 & 51 & 46 & 43 & 50 & 46 & 43 & 49 & 45 & 42 & 48 & 45 & 42 & 1 \\
\hline 7 & $56 \quad 48$ & 43 & 39 & 55 & 47 & 43 & 39 & 46 & 42 & 39 & 45 & 42 & 39 & 45 & 41 & 39 & 7 \\
\hline 8 & 5245 & 40 & 36 & 51 & 44 & 39 & 36 & 43 & 39 & 36 & 42 & 39 & 36 & 42 & 38 & 36 & \\
\hline 0 & $49 \quad 42$ & 37 & 33 & 49 & 41 & 36 & 33 & 40 & 36 & 33 & 40 & 36 & 33 & 39 & 36 & 33 & \\
\hline 1 & $47 \quad 39$ & 34 & 31 & 46 & 39 & 34 & 31 & 38 & 34 & 31 & 37 & 33 & 31 & 37 & 33 & 31 & \\
\hline
\end{tabular}

ALI CANDELA, LUMENS, LUMINANCE, COEFFICIENT OE UTILIZATION AND VCP VALUES IN THIS REPORT ARE BASED ON RELATIVE PHOTOMETRY WHICH ASSUMES A BALLAST FACTOR OF 1.000. ANY CALCULATIONS PREPARED FROM THESE DATA SHOULD INCLUDE AN APPROPRIATE BALLAST FACTOR. 


\section{itl boulder}

FAX (303) 449-5274 INDEPENDENT TESTING LABORATORIES, INC. • 3386 LONGHORN ROAD, BOULDER, COLORADO 80302 • PHONE (303) 442-1255

REPORT NUMBER: ITL40790

PREPARED FOR: U.S. ARMY CORPS OF ENGINEERS

CATALOG NUMBER: DEEP CAN, BLACK BAFFLE

LUMINAIRE: FABRICATED CYLINDRICAL METAL HOUSING WITH BLACK PAINTED

INTERIOR, BLACK PLASTIC LOWER BAFFLE, WHITE PAINTED METAL TRIM RING, OPEN BOTTOM.

LAMP: 150-WATT R40 SPOT INCANDESCENT, RATED 1900 LUMENS, VERTICAL BASE-UP POSITION.

MOUNTING : RECESSED

$\begin{array}{rcr}\text { DEG } & \text { CANDELA } & \text { LUMENS } \\ 0 & 5954 & \\ 5 & 4936 & 418 \\ 15 & 1405 & 403 \\ 25 & 464 & 205 \\ 35 & 88 & 67 \\ 45 & 44 & 32 \\ 55 & 14 & 11 \\ 65 & 0 & 0 \\ 75 & 0 & 0 \\ 85 & 0 & 0 \\ 90 & 0 & \end{array}$

$\begin{array}{lcrr}\text { ZONAL LUMEN } & \text { SUMMARY } & & \\ \text { ZONE } & \text { LUMENS } & \text { 8LAMP } & \text { \&FIXT } \\ 0-30 & 1027 & 54.0 & 90.2 \\ 0-40 & 1094 & 57.6 & 96.2 \\ 0-60 & 1138 & 59.9 & 100.0 \\ 0-90 & 1138 & 59.9 & 100.0 \\ 90-180 & 0 & 0.0 & 0.0 \\ 0-180 & 1138 & 59.9 & 100.0\end{array}$

TOTAL LUMINAIRE EFFICIENCY = $59.9 \%$

CIE TYPE - DIRECT

LUMINAIRE SPACING CRITERION $=0.3$

LUMINOUS DIAMETER: 5.750

LUMINANCE DATA IN CANDELA/SQ M ANGLE AVERAGE MAXIMUM MAX/AVG IN DEG

$\begin{array}{rrrr}45 & 3712 . & 58382 . & 15.7 \\ 55 & 1456 . & 35271 . & 24.3 \\ 65 & 0 . & 643 . & 0.0 \\ 75 & 0 . & 184 . & 0.0 \\ 85 & 0 . & 94 . & 0.0\end{array}$

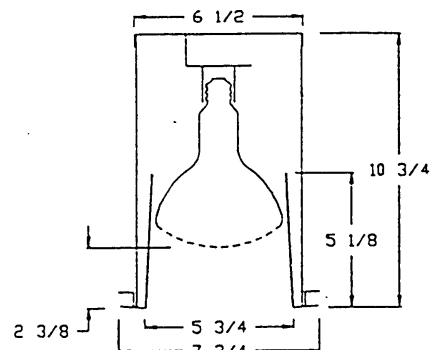




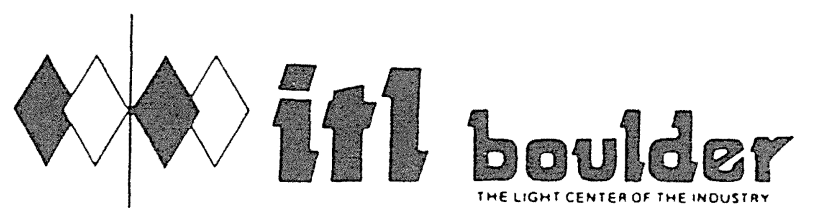

FAX (303) 449-52:: INDEPENDENT TESTING LABORATORIES, INC. • 3386 LONGHORN ROAD, BOULDER, COLORADO 80302 • PHONE (303) 442-125

REPORT NUMBER: ITL40790

DATE: 8-25-1993

PREPARED FOR: U.S. ARMY CORPS OF ENGINEERS

\begin{tabular}{|c|c|c|c|}
\hline \multicolumn{4}{|c|}{$\begin{array}{l}\text { CANDELA DISTRIBUTION } \\
0.0\end{array}$} \\
\hline 0.0 & 5954 & ZONAL LUMEN & SUMMARY \\
\hline 25 & & $0-5$ & 136. \\
\hline $2 \cdot 3$ & 5686 & $5-10$ & 283 \\
\hline 5.0 & 4936 & $10-15$ & 247 . \\
\hline 7.5 & 3949 & $15-20$ & 156. \\
\hline 10.0 & 2951 & $20-25$ & 115. \\
\hline 12.5 & 2083 & $25-30$ & 91. \\
\hline 15.0 & 1405 & $30-35$ & 46. \\
\hline 17.5 & 946 & $35-40$ & 21. \\
\hline 20.0 & 681 & $40-45$ & 18. \\
\hline 22.5 & 546 & $45-50$ & 14. \\
\hline 25.0 & 464 & $50-55$ & 10. \\
\hline 27.5 & 358 & $55-60$ & 2 . \\
\hline 30.0 & 228 & $60-65$ & 0 . \\
\hline 32.5 & 157 & $65-70$ & 0. \\
\hline 35.0 & 88 & $70-75$ & 0 \\
\hline 37.5 & 63 & $75-80$ & 0 \\
\hline 40.0 & 55 & $80-85$ & 0 \\
\hline 42.5 & 49 & $85-90$ & 0. \\
\hline 45.0 & 44 & & \\
\hline 47.5 & 35 & & \\
\hline 50.0 & 28 & & \\
\hline 52.5 & 22 & & \\
\hline 55.0 & 14 & & \\
\hline 57.5 & 4 & & \\
\hline 60.0 & 0 & & \\
\hline 62.5 & 0 & & \\
\hline 65.0 & 0 & & \\
\hline 67.5 & 0 & & \\
\hline 70.0 & 0 & & \\
\hline 72.5 & 0 & & \\
\hline 75.0 & 0 & & \\
\hline 77.5 & 0 & & \\
\hline 80.0 & 0 & & \\
\hline 82.5 & 0 & & \\
\hline 85.0 & 0 & & \\
\hline 87.5 & 0 & & \\
\hline 90.0 & 0 & & \\
\hline
\end{tabular}




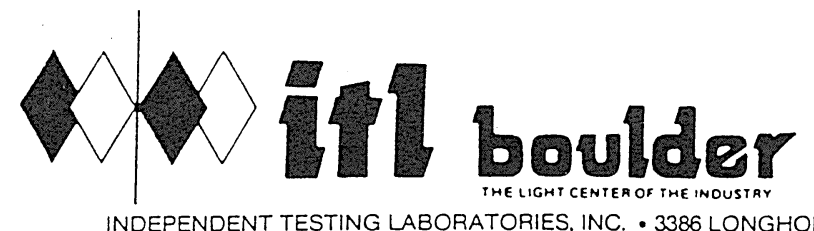

INDEPENDENT TESTING LABORATORIES, INC. • 3386 LONGHORN ROAD, BOULDER. COLORADO 80302 • PHONE (303) 442-i 25
$\operatorname{FAX}(303) 449-52 T:$

DATE : $8-25-1993$

REPORT NUMBER: ITL40790

PREPARED FOR: U.S. ARMY CORPS OF ENGINEERS

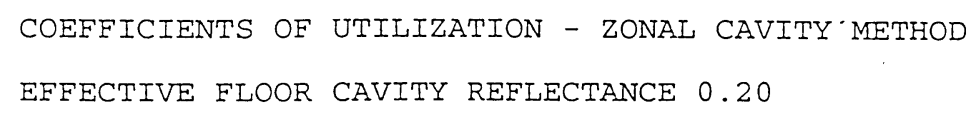

\begin{tabular}{|c|c|c|c|c|c|c|c|c|c|c|c|c|c|c|c|c|c|c|}
\hline $\mathrm{RC}$ & \multicolumn{4}{|c|}{80} & \multicolumn{4}{|c|}{70} & \multicolumn{3}{|c|}{50} & \multicolumn{3}{|c|}{30} & \multicolumn{3}{|c|}{10} & 0 \\
\hline$R W$ & 70 & 50 & 30 & 10 & 70 & 50 & 30 & 10 & 50 & 30 & 10 & 50 & 30 & 10 & 50 & 30 & 10 & 0 \\
\hline 0 & 71 & 71 & 71 & 71 & 70 & 70 & 70 & 70 & 66 & 66 & 66 & 64 & 64 & 64 & 61 & 61 & 61 & 60 \\
\hline 1 & 69 & 67 & 66 & 65 & 67 & 66 & 65 & 64 & 64 & 63 & 62 & 62 & 61 & 60 & 59 & 59 & 59 & D \\
\hline 2 & 66 & 64 & 62 & 61 & 65 & 63 & 61 & 60 & 61 & 60 & 59 & 59 & 58 & 57 & 58 & 57 & 56 & 5 \\
\hline 3 & 64 & 61 & 59 & 57 & 63 & 60 & 58 & 57 & 59 & 57 & 56 & 57 & 56 & 55 & 56 & 55 & 54 & 3 \\
\hline 4 & 62 & 59 & 56 & 54 & 61 & 58 & 56 & 54 & 57 & 55 & 53 & 56 & 54 & 53 & 55 & 53 & 52 & I \\
\hline 5 & 60 & 56 & 54 & 52 & 59 & 56 & 53 & 51 & 55 & 53 & 51 & 54 & 52 & 51 & 53 & 51 & 50 & 50 \\
\hline 6 & 58 & 54 & 52 & 50 & 57 & 54 & 51 & 49 & 53 & 51 & 49 & 52 & 50 & 49 & 51 & 50 & 49 & 48 \\
\hline 7 & 56 & 52 & 50 & 48 & 56 & 52 & 49 & 48 & 51 & 49 & 47 & 51 & 49 & 47 & 50 & 48 & 47 & 46 \\
\hline 8 & 55 & 51 & 48 & 46 & 54 & 50 & 48 & 46 & 50 & 47 & 46 & 49 & 47 & 46 & $\Delta 9$ & 47 & 46 & 5 \\
\hline 9 & 53 & 49 & 46 & 45 & 53 & 49 & 46 & 45 & 48 & 46 & 45 & 48 & 46 & 44 & 47 & 46 & 44 & $=$ \\
\hline 10 & 52 & 48 & 45 & 43 & 51 & 47 & 45 & 43 & 47 & 45 & 43 & 47 & 45 & 43 & 46 & 44 & 43 & \\
\hline
\end{tabular}

ALL CANDELA, LUMENS, LUMINANCE, COEFFICIENT OF UTILIZATION AND VCP VALUES IN THIS REPORT ARE BASED ON RELATIVE PHOTOMETRY W:ICH ASSUMES A BALLAST FACTOR OF 1.000. ANY CALCULATIONS PREPARED FROM THESE DATA SHOULD INCLUDE AN APPROPRIATE BALLAST FACTOR. 


\section{USACERL DISTRIBUTION}

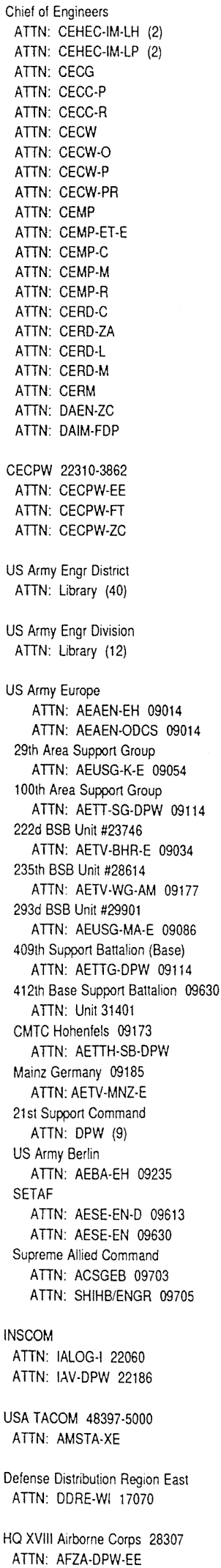

\author{
4th Infantry Div (MECH) $80913-5000$ \\ ATTN: AFZC-FE \\ US Army Materiel Command (AMC) \\ Alexandria, VA 22333.0001 \\ ATTN: AMCEN-F \\ Installations: (20) \\ FORSCOM \\ Forts Gillem \& McPherson 30330 \\ ATTN: FCEN \\ Installations: (22) \\ 6th Infantry Division (Light) \\ ATTN: APVR-DE 99505 \\ ATTN: APVR-WF-DE 99703 \\ TRADOC \\ Fort Monroe 23651 \\ ATTN: ATBO-G \\ Installations: (20) \\ Fort Belvoir 22060 \\ ATTN: CETEC-IM-T \\ ATTN: CETEC-ES 22315-3803 \\ ATTN: Water Resources Support Ct \\ USA Natick RD\&E Center 01760 \\ ATTN: STRNC-DT \\ ATTN: DRDNA-F \\ US Army Materials Tech Lab \\ ATTN: SLCMT-DPW 02172 \\ USARPAC 96858 \\ ATTN: DPW \\ ATTN: APEN-A \\ SHAPE 09705 \\ ATTN: Infrastructure Branch LANDA \\ Area Engineer, AEDC-Area Office \\ Arnold Air Force Station, TN 37389 \\ HQUSEUCOM 09128 \\ ATTN: ECJ4-LIE \\ AMMRC 02172 \\ ATTN: DRXMR-AF \\ ATTN: DRXMR-WE \\ CEWES 39180 \\ ATTN: Library \\ CECRL 03755 \\ ATTN: Library \\ USA AMCOM \\ ATTN: Facilities Engr 21719 \\ ATTN: AMSMC.EH 61299 \\ ATTN: Facilities Engr (3) 85613 \\ USAARMC 40121 \\ ATTN: ATZIC-EHA \\ Military Traffic Mgmt Command \\ ATTN: MTEA.GB.EHP 07002 \\ ATTN: MT-LOF 20315 \\ ATTN: MTE-SU.FE 2846 \\ ATTN: MTW.IE 94626 \\ Fort Leonard Wood 65473 \\ ATTN: ATSE-DAC-LB (3) \\ ATTN: ATZA-TE-SW \\ ATTN: ATSE-CFLO \\ ATTN: ATSE-DAC-FL \\ ATTN: Australian Liaison Office
}

Military Dist of WASH
Fort MCNair

ATTN: ANEN 20319

USA Engr Activity, Capital Area ATTN: Library 22211

US Army ARDEC 07806-5000 ATTN: AMSTA-AR-IMC

Engr Societies Library ATTN: Acquisitions 10017

Defense Nuclear Agency ATTN: NADS 20305

Defense Logistics Agency ATTN: DLA-WI 22304

Walter Reed Army Medical Ctr 20307

National Guard Bureau 20310 ATTN: NGB-ARI

US Military Academy 10996 ATTN: MAEN-A

ATTN: Facilities Engineer ATTN: Geography \& Envr Engrg

Naval Facilities Engr Command ATTN: Facilities Engr Command (8) ATTN: Division Otfices (11) ATTN: Public Works Center (8) ATTN: Naval Constr Battalion Cir 93043 ATTN: Naval Facilities Engr Service Center 93043-4328

8th US Army Korea ATTN: DPW (12)

USA Japan (USARJ)

ATTN: APAJ.EN-ES 96343

ATTN: HONSHU 96343

ATTN: DPW-Okinawa 96376

416th Engineer Command 60623

ATTN: Gibson USAR Ctr

US Army HSC

Fort Sam Houston 78234

ATTN: HSLO-F

Fitzsimons Army Medical Cir

ATTN: HSHG-DPW 80045

Tyndall AFB 32403

ATTN: HQAFCESA Program OfC

ATTN: Engrg \& SNo Lab

USA TSARCOM 63120

ATTN: STSAS.F

American Public Works Assoc. 64104-1806

US Army CHPPM

ATTN: MCHB.DE 21010

US Gov't Printing Office 20401

ATTN: Rec Sec/Deposit Sec (2)

Nat'l Institute of Standards \& Tech ATTN: Library 20899

Defense Tech Info Center 22304 ATTN: DTIC-FAB (2) 
DEPARTMENT OF THE ARMY
CONSTRUCTION ENGINEERING RESEARCH LABORATORIES

CORPS OF ENGINEERS

PO BOX 9005

CHAMPAIGN, ILLINOIS 61826-9005

BULK RATE

US POSTAGE

PAID

CHAMPAIGN IL
PERMIT NO. 871

OFFICIAL BUSINESS 